\title{
DESIGN AND VALIDATION OF A SOFT SWITCH FOR A VIRTUALLY VARIABLE DISPLACEMENT PUMP
}

\author{
A THESIS \\ SUBMITTED TO THE FACULTY OF THE GRADUATE SCHOOL \\ OF THE UNIVERSITY OF MINNESOTA \\ BY
}

Brandon Beckstrand

IN PARTIAL FULFILLMENT OF THE REQUIREMENTS

FOR THE DEGREE OF

Master of Science in Mechanical Engineering

Dr. James D. Van de Ven

June, 2015 
(C) Brandon Beckstrand 2015

ALL RIGHTS RESERVED 


\section{Acknowledgments}

I would like to thank my Advisor, Dr. James Van de Ven, for allowing me the opportunity to embark on this adventure, and for his patience and guidance as I continue to learn how to be an engineer. The people of the MEPS lab for spending their time giving helpful suggestions, having fun, and laughing even when things don't go well. My wife for putting up with my engineering jokes, listening to me and smiling while I excitedly explain things that are either not interesting or go over her head, and sacrificing so much of her time and energy to allow me to focus on things that need to be done. My kids for understanding (even if it is only a little bit) that, "Daddy needs to work on his thesis now," and being patient with me as I have to call rain checks on fun activities. And lastly my parents and siblings who taught me the importance of education, how to work hard and play hard, and who believe in me at times when I don't believe in myself. Thank you all! 


\section{Abstract}

Switch-mode hydraulic control is a compact and theoretically efficient alternative to throttling valve control or variable displacement pump control. The virtually variable displacement circuit created by a pulse-width modulated high-speed valve has drawbacks, such as large energy losses due to throttling and compressibility during valve transitions. Hydraulic soft switching was proposed as method of reducing the throttling energy loss, by absorbing, in a small variable volume chamber, the flow that would normally be throttled across the transitioning high speed valve. An active locking mechanism was presented by Van de Ven that overcomes the main challenge with soft switching, which is a lock mechanism that releases quickly and with precise timing.

A numerical model is developed for a switch-mode pumping circuit, utilizing the proposed soft switch. The model is then used as a means of designing a proof of concept prototype to further verify the model and provide a future optimization tool. The prototype design includes methods for controlling the soft switch spring preload, travel distance, piston displacement required to unlock the soft switch, valve command duty ratio, switching cycle length, and load pressure. Testing demonstrated that the soft switch circuit performed as expected within a set of parameter values, defining normal operation. High control valve switching periods, high load pressures, high flow rates, low soft switch unlocking distances, and low spring preloads caused the soft switch to unlock prematurely in the switching cycle. This resulted in the soft switch to failing to absorb the switched volume pressure spike during control valve transitions, leading to low efficiencies.

Under normal operating conditions, the soft switch circuit was calculated to be $60.9 \%$ efficient, which is lower than the $62.1 \%$ efficiency for same circuit without a soft switch, and also lower than the $70.5 \%$ efficiency predicted by the numerical model. The discrepancies between the numerical and experimental model can be attributed to poor resolution in the measurement instruments, as well as the model not accurately capturing flow dynamics through the check valves behind the soft switch piston. Since the soft switch parameters were chosen to validate the lock release concept, and not to maximize efficiency, it was not unexpected that the baseline soft switch case had lower efficiency than the control case.

Using the insights gained from these model comparisons, an efficient locking soft switch can be designed for use in a dual soft switch circuit scheme, shown by Van de Ven to reduce energy losses by $66 \%$ compared to a control circuit. A successful hydraulic soft switch circuit will allow for the use of slower switching valves, which will reduce component cost as well as energy consumption. 


\section{Contents}

Acknowledgments $\quad$ i

Abstract

List of Tables $\quad$ V

List of Figures $\quad$ vi

1 Introduction 1

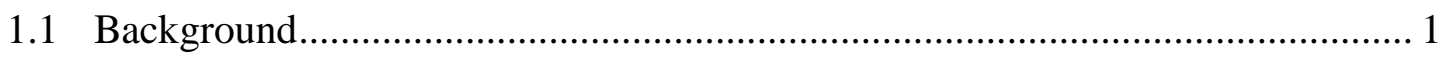

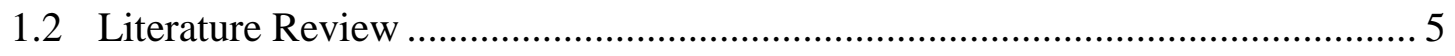

1.2.1 PWM Circuits .............................................................................. 5

1.2.2 Transitional Throttling Loss ......................................................... 9

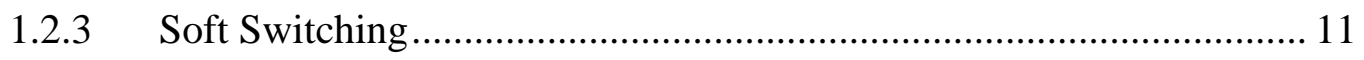

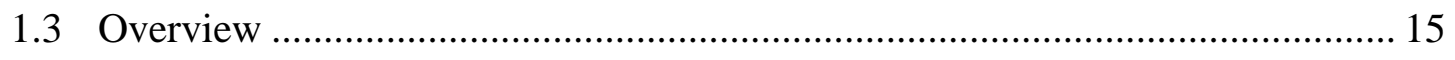

2 Numerical Model 16

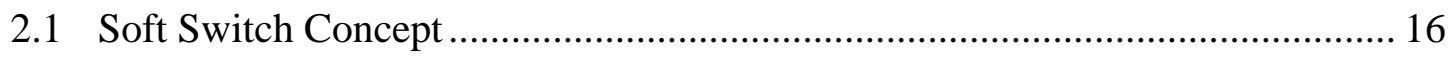

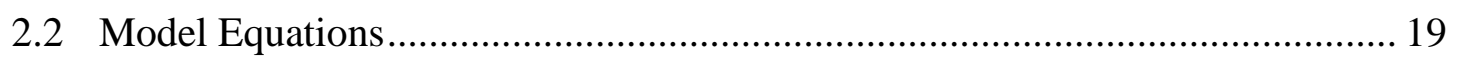

2.2.1 Port Positions and Component Areas............................................. 19

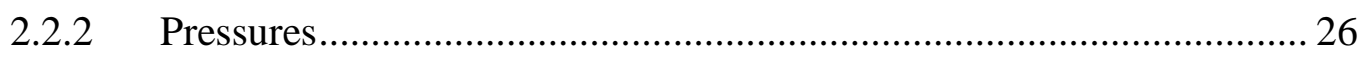

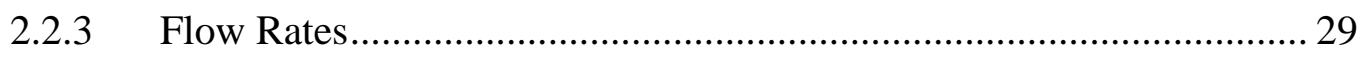

2.2.4 Component Positions .............................................................. 34

2.3 Model Architecture and Functionality ...................................................... 38

iii 
2.4 Model Development Conclusion .............................................................. 39

3 Physical Model Development 41

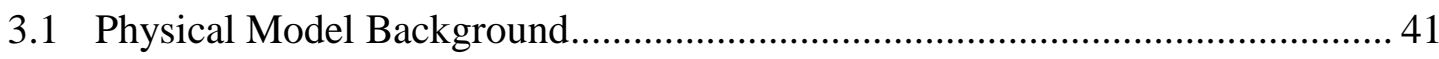

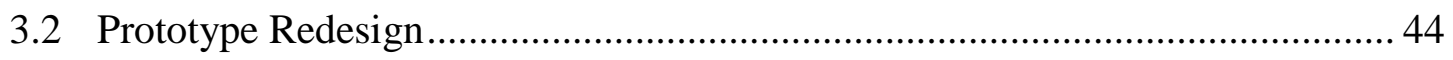

3.2.1 Piston and Sleeve Design ..................................................... 45

3.2.2 Spring, Limits, and Check Valves .............................................. 49

3.2.3 Circuit and Manifold Design...................................................... 52

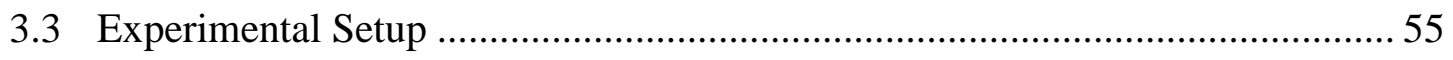

3.3.1 Components and Layout …..................................................... 55

3.3.2 System Control and Data Acquisition.......................................... 59

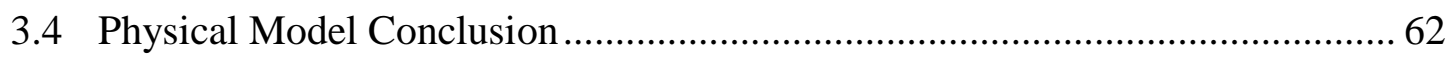

4 Simulation and Experiment Results 63

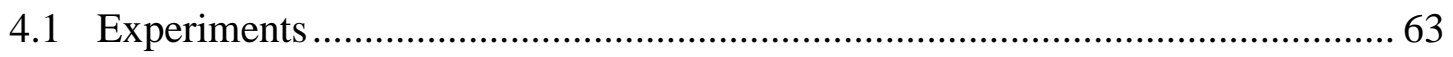

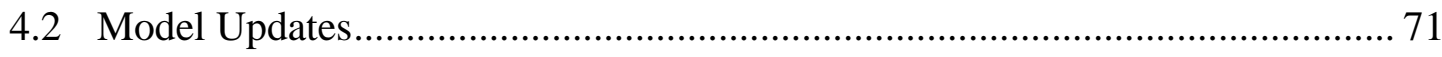

4.2.1 Model Robustness and Parameter Updates ................................... 71

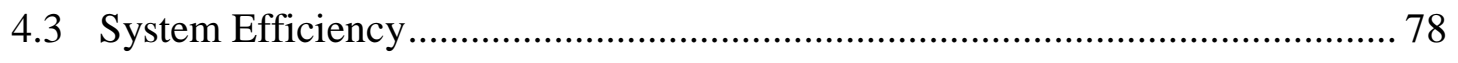

4.4 Simulation and Experiment Results Conclusion ....................................... 81

5 Conclusion $\quad 82$

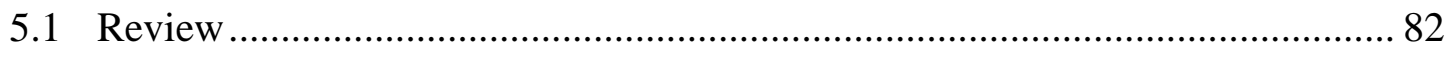

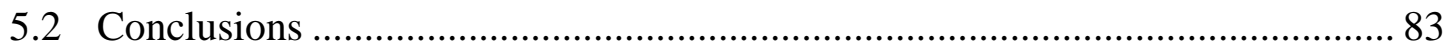

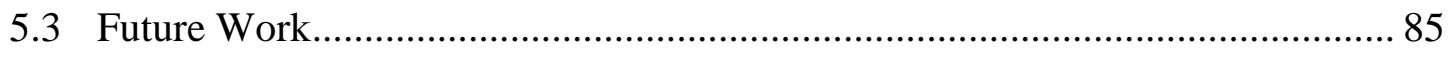

$\begin{array}{ll}\text { Bibliography } & 87\end{array}$

$\begin{array}{ll}\text { Appendices } & 91\end{array}$ 


\section{List of Tables}

2.1 - The parameters required to calculate the port positions and open areas................. 25

2.2 - The parameters required to calculate the system pressures ................................. 29

2.3 - The parameters required to calculate the flow rates ......................................... 34

2.4 - The parameters required to calculate the accelerations ...................................... 37

3.1 - Triana's optimized soft switch parameters ....................................................... 43

3.2 - Component part numbers and specifications for soft switch test bed..................... 57

4.1 - Operating parameters of significant interest for the physical model ...................... 64

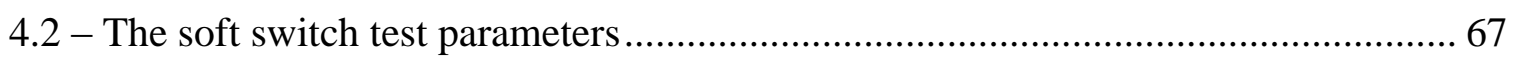

4.3 - Switched volume pressure spike for the adjusted parameters ............................. 70

4.4 - System parameter updates to bring the numerical model into agreement .............. 76

4.5 - The efficiency of the experiment and numerical model ..................................... 78 


\section{List of Figures}

1.1 - A simple bleed off hydraulic control circuit ....................................................... 2

1.2 - A simple virtually variable displacement pump (VVDP) ......................................... 4

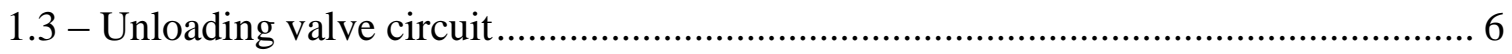

1.4 - DC-DC buck converters.............................................................................. 9

1.5 - DC-DC boost converter with soft switch capacitance elements included ................ 11

1.6 - Soft switch concept as proposed by Rannow and $\mathrm{Li}$............................................ 12

1.7 - Diagram of the virtually variable displacement pump locking soft switch ............. 14

2.1 - Soft switch concept as proposed by Van de Ven ....................................................... 17

2.2 - Virtually variable displacement pumping circuit..................................................... 18

2.3 - Graphical representation of high speed valve spool displacement .......................... 20

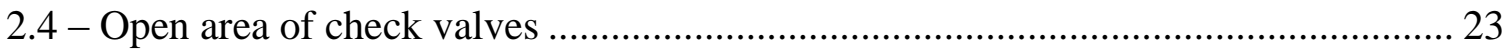

2.5 - Open area of the soft switch internal port to tank .................................................. 24

2.6 - High speed valve command signal, spool displacement, and orifice areas ............. 26

2.7 - Pressures, flow rates, and displacements of interest .............................................. 27

2.8 - Leakage flow paths past the soft switch piston...................................................... 32

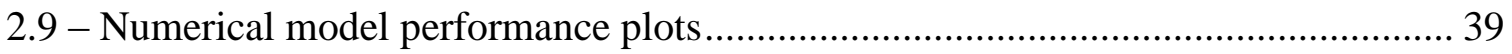

3.1 - Initial soft switch lock-release prototype ……………………………………...... 42

3.2 - Initial soft switch lock-release prototype circuit................................................... 43

3.3 - Cross-sectional view of the assembled soft switch.................................................. 45

3.4 - The soft switch sleeve tank and load ports ........................................................... 46

3.5 - The left end of the soft switch sleeve..................................................................... 47

3.6 - Cross-sectional view of the soft switch piston.................................................... 48

3.7 - Energy loss through internal piston ports for one switching cycle .......................... 49

3.8 - The face plate of the soft switch manifold ................................................................ 50 
3.9 - Check valve seated within the proper check valve housing .................................... 51

3.10 - The bleeding sequence for the soft switch manifold ............................................ 53

3.11 - Cross-sectional view of the soft switch manifold ................................................. 54

3.12 - Soft switch manifold viewed from the reverse side............................................ 55

3.13 - Soft switch test bed setup with component labels .................................................. 56

3.14 - The soft switch hydraulic test circuit layout .................................................... 58

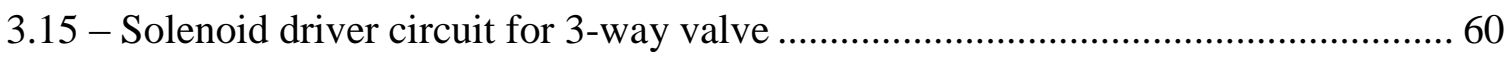

3.16 - Magnetoresistive position sensor orientation in relation to a magnet..................... 61

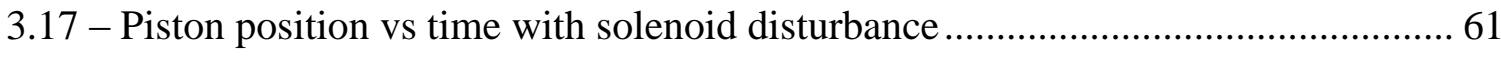

4.1 - Soft switch piston displacement and pressure profiles for control case .................. 65

4.2 - Soft switch piston displacement and pressure profiles for baseline parameters ...... 66

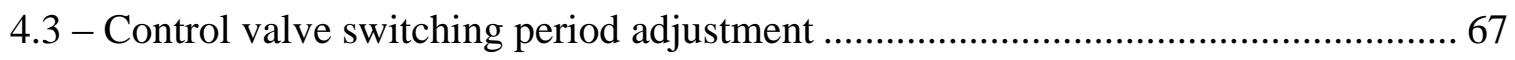

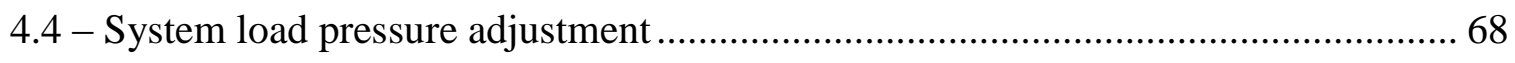

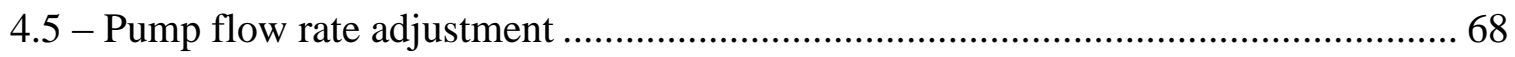

4.6 - Soft switch unlocking distance adjustment............................................................. 69

4.7 - Soft switch spring preload adjustment .................................................................... 69

4.8 - Comparison between the numerical model and the baseline test case...................... 71

4.9 - Comparison between the original design model, updated model ........................... 74

4.10 - Comparison between the baseline case and the numerical model updates............ 75

4.11 - The numerical model predicting soft switch piston behavior for the high ............ 77

4.12 - The numerical model predicting soft switch piston behavior for the low ............. 77

4.13 - The efficiencies of throttling control, PWM without soft switch ......................... 80

A.1 - Detail design for the soft switch manifold ............................................................ 93

A.2 - Detail design for the soft switch face plate .............................................................. 94

A.3 - Detail design for soft switch piston................................................................. 95

A.4 - Detail design for soft switch sleeve ................................................................ 96 


\section{Chapter 1:}

\section{Introduction}

\subsection{Background}

A hydraulic circuit is a combination of hydraulic fluid conductors, valves, and actuators that are used to perform some function. A pump, driven by a prime mover, provides fluid displacement to the system, frequently to an actuator, which then performs some useful physical task. The motion of the actuator can be manipulated at the pump, via volumetric displacement control, or away from the pump using valve control. This manipulation allows the actuator to receive either a desired pressure or flow rate from the pump.

Since the historical emphasis of hydraulic circuit design has been on performance, not efficiency, most hydraulic systems throttle pump flow to achieve a reduction in load line pressure. A simple hydraulic control circuit, referred to as a bleed-off circuit, uses a fixed displacement pump and a flow control valve in parallel with the load portion of the circuit as shown in Fig. 1.1. As the control valve is incrementally closed, the area for fluid to flow to tank decreases which causes the pressure sensed at the load to increase and vice versa. This circuit is cheap, simple, and effective controlling flow or pressure in steady-state conditions, but it is fairly inefficient because some or all of the flow from the pump is throttled across the control valve. 


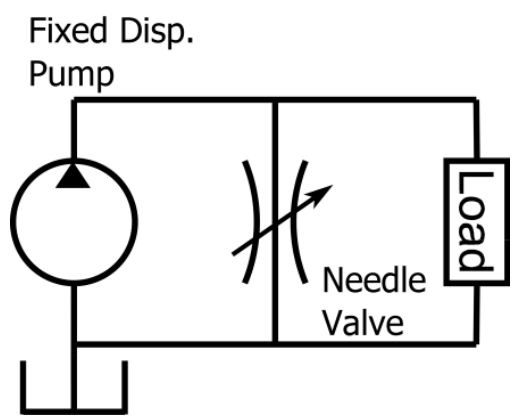

\section{Figure 1.1 - A simple bleed off hydraulic control circuit, which carries the disadvantage of} energy lost as excess flow is throttled across a valve to tank.

The most common type of pump for fluid power systems is the positive displacement pump. Assuming constant shaft speed, and neglecting small internal leakage, the positive displacement pump delivers a constant output flow. Two subcategories of positive displacement pumps are the fixed and variable displacement pumps. One of the most inexpensive widely available pump architectures is the fixed displacement gear pump. A gear pump operates by carrying fluid between the teeth of a set of meshed gears. Because of its compact design with few components, gear pumps are regularly used in situations where a fixed displacement is desired. The primary variable displacement pump architectures are vane and piston pumps. A piston pump operates by rotating a barrel of pistons and cylinders where the ends of the pistons ride against an angled swash plate. This rotation causes the pistons to reciprocate as they travel around the shaft of the pump. As they are pulled back they draw fluid from the inlet, and as they are pushed forward they force fluid through the outlet. This is made a variable displacement design by allowing the angle of the swash plate, which the piston slippers ride on, to be adjusted thereby changing the length of the piston stroke [1].

Varying the displacement of a pump to control an actuator is a more efficient way to control a hydraulic circuit than valve control, because it eliminates the energy loss that occurs when fluid is throttled across a control valve. Unfortunately, variable displacement pumps are more complex and can be up to three times larger and heavier than fixed displacement pumps for the same displacement and are therefore more 
expensive and inconvenient from a size perspective [2]. The associated displacement control can also take significant energy to adjust (either manually, or by servo or compensator), and has a typical neutral to full stroke response times on the order of 1 second, which can make it impractical in applications requiring constant adjustment and fast displacement control [2]. On the other hand, control valves have response times on the order of milliseconds, which is easily adequate for most applications [2,3].

In a complicated system with multiple hydraulic actuators each with its own load requirements, the common control approach utilizes a central hydraulic power supply with each individual actuator being controlled by its own control valve. The BigDog robot, designed by Boston Dynamics, is a quadruped robot that uses one hydraulic power supply to control all of the leg actuators [4]. This approach easily meets the energy requirements of the system and allows for precise control, but the throttling losses associated with that approach make it an inefficient solution, increasing the engine size requirement and the fuel consumed. In fact, fluid power systems in the U.S. have an average efficiency of $22 \%$, due in large part to throttling losses [5].

Another control approach provides a dedicated variable displacement pump to each actuator. This displacement-controlled actuator approach has been implemented on an excavator at Purdue University where it was found to give a $40 \%$ fuel savings over the standard load-sensing control where 50\% of the system energy was lost to throttling [68]. Because of the cost and size of such a system, it is usually only feasible in applications like construction equipment where the added system mass is not detrimental to system operation. Many systems that utilize hydraulic power, such as robotics and aerospace, have system mass as a limiting factor and therefore need actuation schemes with higher power densities than can be achieved with this displacement-control approach.

An alternative control architecture, the focus of this thesis, uses rapid on and off switching of pump flow via a high speed valve. This approach is called pulse-widthmodulation (PWM) and is analogous to switch mode control in power electronics [9]. A simple hydraulic switch mode circuit utilizes a fixed displacement pump and a high speed on/off valve to create a virtually variable displacement pump (VVDP) as seen in Fig. 1.2 
$[2,10-12]$. When the on/off valve is switched on, the full flow of the pump is sent to the load, and when it is off, the flow is sent to the tank. In both valve positions the system experiences minimal throttling losses as the valve is fully open. By varying the percentage of time the on/off valve is switched to load (on), which is called the duty ratio, the power sent to load can be adjusted. One of the main advantages of VVDPs is that the initial component cost is much lower than that of a variable displacement pump.

Throttling losses are minimized when the high speed valve is fully on or fully off, but when the high speed valve is transitioning, fluid is still being throttled across the high speed valve and possibly the relief valve.

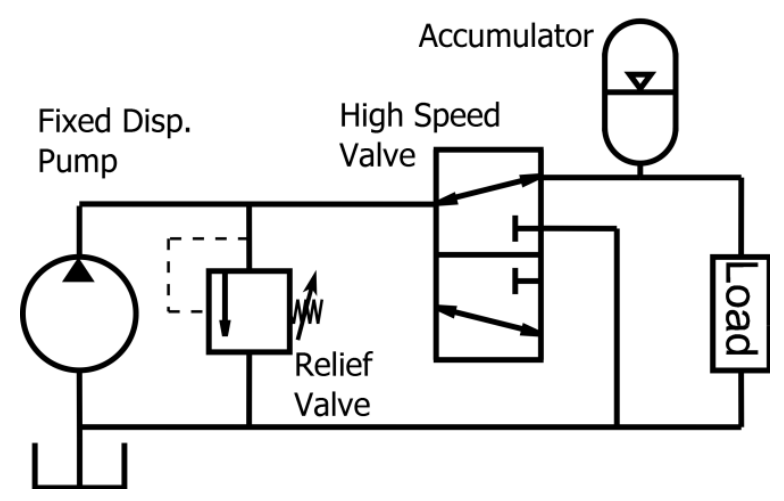

Figure 1.2 - A simple virtually variable displacement pump (VVDP). The high speed valve rapidly switches back and forth alternately sending fluid to load then tank. The accumulator smoothes out the load line switching pulses.

One method of minimizing the throttling energy loss during valve transitions is to absorb the normally throttled fluid in a small piston cylinder device called a soft switch. The soft switch is designed to absorb flow when the valve is transitioning and release the fluid when the valve is fully open. The goal of the research presented in this paper is to develop a numerical model of a self-actuating soft switch and validate the model experimentally, allowing the model to be used for future design and optimization work. 


\subsection{Literature Review}

Variable displacement pumps address some of the basic needs of hydraulic systems and may help avoid the use of throttling valves to control system flow rates. Alternatively, digital hydraulic systems are being developed that allow smaller and cheaper fixed displacement pumps to be used efficiently in a circuit requiring variable displacement control [13]. This literature review will address the need for, and advantages and disadvantages of, digital hydraulic systems, with a focus on transitional throttling losses. Hydraulic analogies of electronic PWM circuits will be discussed, as well as the benefits of the adding soft switches to those circuits.

\subsubsection{PWM Circuits}

An unloading valve in conjunction with a fixed displacement pump allows a system to build to a desired pressure and then have excess fluid sent to tank at low pressure (Fig. 1.3). With the control valve switched off, pressure builds in the load line and accumulator until the pressure setting of the unloading valve is reached. Pump flow is then diverted to the tank through the unloading valve at low pressure. The check valve allows pressurized fluid to remain in the accumulator. When the control valve switches on, the accumulator begins to discharge and the drop in pressure closes the unloading valve, causing the fluid to flow to load and the pressure to build again. When low flow is demanded at the load, this arrangement can be more efficient than a comparable variable displacement pump [14]. 


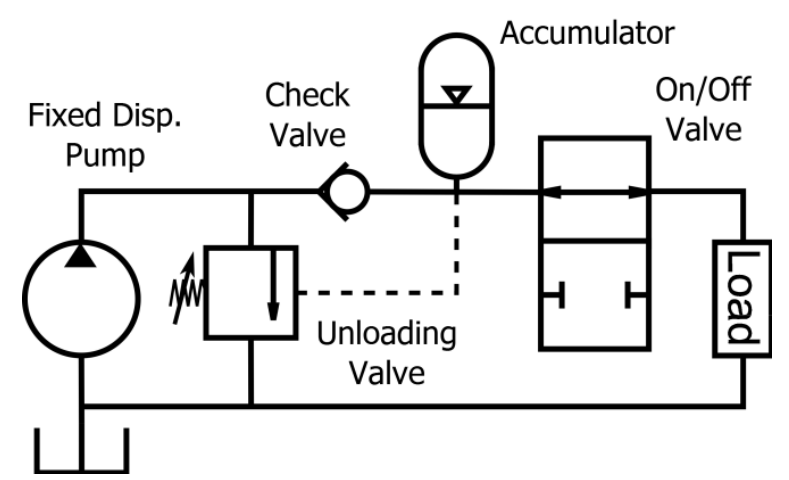

Figure 1.3 - Unloading valve circuit. When desired load pressure is reached, the unloading valve sends the excess flow to tank.

A physical system was created by Tomlinson and Burrows to verify the practicality of controlled unloading of a fixed-displacement pump [15]. It was discovered that when the unloading valve was controlled correctly (cut-in and cut-out pressure adjustments) the system was able to maintain the desired demand pressure with minimal pressure oscillation through a range of pump flow rates. This research proved that switching control is possible, though this approach had slow response times, required pressure adjustments when loading conditions changed, and became unstable with changes in fluid temperature.

Frequently a single hydraulic pump is used to drive multiple actuators in a hydraulic circuit. Common practice dictates the pump be sized to provide the maximum flow rate and pressure needed by all actuators simultaneously, which is an inefficient approach under normal operating conditions. Cao et al. suggested the use of switch-mode hydraulics to drive multiple actuators from a main hydraulic power supply, with each of the actuators being controlled by high speed switching valves [16]. This decreased the overall cost of the system by removing the need for multiple pumps. In one arrangement, each high speed valve rapidly switched back and forth between sending flow to an actuator to sending flow to tank. If the high speed valve is in the tank position for $40 \%$ of the time, the system pressure should effectively decreases from $10 \mathrm{MPa}$ to $6 \mathrm{MPa}$. The efficiency of this system only reached $58 \%$, which, though not high, is still more efficient than throttling valve control. Beachley and Fronczak suggested instead a multi-branch computer controlled sequential apportioning circuit [17]. An adequate cycle time and 
pump size was chosen, and each branch of the circuit was opened to the pump sequentially for a varying percentage of the cycle. This approach solved the problem of the pump having to constantly provide maximum pressure and flow rate, thereby increasing system efficiency.

Other research has been done regarding pulse-width-modulation (PWM) of high speed solenoid valve control approaches. Barth et al. created an active and stable control strategy using PWM to determine system flow rate in a pneumatic system [18]. By creating a continuous linear control model with good tracking of a physical system, the need for proportional control valves was removed. The removal of proportional control valves as well as pressure sensors that would normally be required for feedback control reduced the implementation costs by an order of magnitude. PWM circuits can also be run in ballistic mode as demonstrated by Flor et al. [19], using a technique patented by Mehling and Weiler [20]. This technique increased the flow resolution of a system by switching a high speed valve in a pattern that allowed for partial opening of the valve before reseating. Though this research showed that good control can be achieved in PWM circuits, and that they can be versatile in their control schemes, little concern was given to the efficiency of the systems. The circuits suffered from fluid compressibility and throttling losses across the control valves, especially in the case of the solenoid valve used in ballistic mode, since it did not regularly reach a fully open position.

As problems with overall system functionality and control of PWM circuits were addressed, more time was spent determining the magnitudes of the energy loss mechanisms. Batdorff and Lumkes outlined the functionality and advantages of a virtually variable displacement pump (VVDP), which is similar to the PWM circuits previously outlined [11]. Aside from modeling the system, they also discovered that a VVDP is capable of maintaining a constant pressure rail, as long as the PWM frequency is low (less than $10 \mathrm{~Hz}$ ) and the system accumulator is large. Perhaps most importantly, they related that the dominant power loss in VVDPs will usually be throttling losses when the high speed PWM valve is in transition. A switch-mode circuit concept by Rannow et al. was tested under various duty ratios and switching frequencies to determine their effects on system efficiency [10]. They found that as switching frequency 
increases, compressibility losses increase linearly and efficiency decreases. It was further proven that decreasing control valve transition times decreases throttling losses. The finding that transitional throttling losses are a large part of PWM system losses has led to both valve and circuit redesign.

One of the redesign strategies being studied draws further inspiration from power electronics. The electronic DC-DC buck converter (Fig. 1.4a) is a circuit that varies the duty ratio of a switch to supply a lower output voltage than the input voltage to the switch [9]. De Negri et al. showed that there are direct analogies between electric and hydraulic circuit components, such as electrical power supplies and hydraulic pumps, diodes and check valves, capacitors and accumulators, and transistors and check valves [21]. By constructing and testing a hydraulic buck converter (Fig. 1.4b), comparable to an electric buck converter, they further showed that the linear modelling of electronic buck converter circuits also applies to hydraulic circuit prediction. Guglielmino et al., also using the DC-DC buck converter as inspiration, created a hydraulic switching converter [22]. Operating their system at a frequency of $80 \mathrm{~Hz}$ and duty ratio in the range of 20$60 \%$, in both flow control and pressure control configurations, they realized approximately $75 \%$ energy savings over proportional valve resistive control. Though these converters did little to address the differences in hydraulic and electronic flow momentum, which leads to considerable throttling and compressibility losses throughout the hydraulic system, electric and hydraulic converter comparison was realized, opening the door for future circuit comparison. 


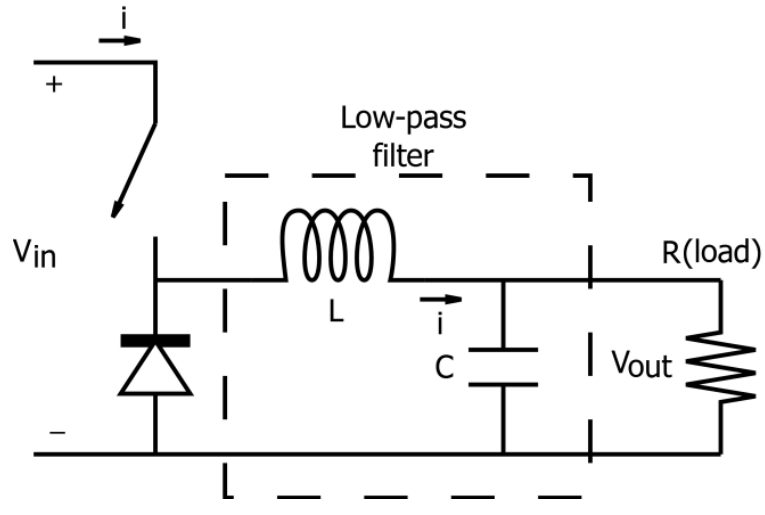

(a) Electronic DC-DC buck converter. Adapted from [9]

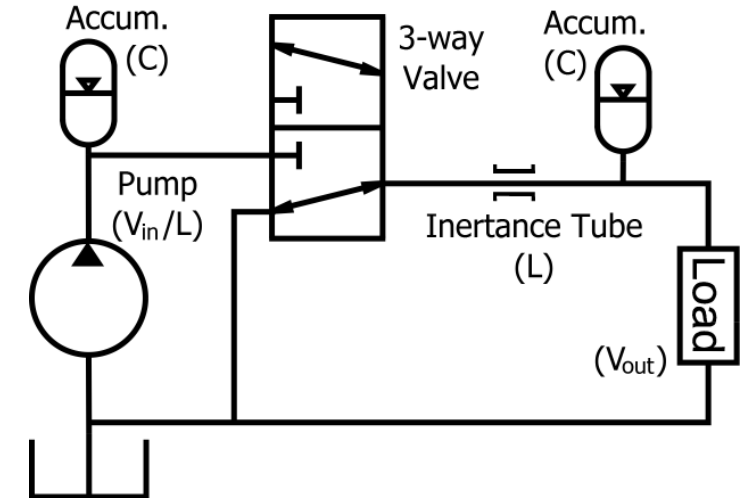

(b) Hydraulic DC-DC buck converter. Adapted from [21]

Figure 1.4 - DC-DC buck converters, designed to convert a high input power (voltage or pressure) to a lower and near constant output power via PWM and filtering.

\subsubsection{Transitional Throttling Loss}

The wide spread interest in PWM research confirms the potential of the technology as well as affirming that throttling losses across the control valve during valve transitions are a main power loss mechanism of the system. Research then turned to quantifying transitional throttling losses and minimizing them through better models and valve designs.

In a bid to minimize energy losses in high speed PWM valves Kudzma et al. designed a linear high speed valve with low leakage, low flow resistance, and high flow gain [23]. The design decreases transitional throttling losses by moving the valve spool a short distance which opens multiple flow passages simultaneously in a fraction of a millisecond. The valve is designed to allow a flow rate of $65 \mathrm{Lpm}$ at a pressure drop of 1 $\mathrm{MPa}$. Prototypes of this valve have failed to operate as predicted due to poor machining tolerance and valve expansion due to fluid heating.

Others like Katz and Van de Ven sought to solve high speed valve control and slow actuation speed by designing a rotary valve with a variable duty ratio [24-26]. Numerical valve simulation showed that at a duty ratio of $25 \%$, the total valve losses 
were broken down as follows: throttling $38.7 \%$, leakage $34.4 \%$, viscous friction $15.3 \%$, and fluid compressibility $11.9 \%$. Though the prototype failed to realize these values due to machining problems, component misalignment and bearing friction, it is clear to see that throttling losses are of huge concern even in high speed rotary valve design. A redesign by $\mathrm{Wu}$ and Van de Ven predicted that the four system power losses would be exactly opposite in importance than the initial design suspected, with compressibility losses being $33 \%$, viscous friction being $29.8 \%$, leakage being $22.8 \%$, and throttling being $14.5 \%$ of the total energy loss [27]. With no record of the building and testing of this redesign, it is hard to say whether these predictions were accurate.

Another high speed rotary valve design by Tu et al. culminated in a valve capable of switching speeds less than $3 \mathrm{~ms}$ [28-29]. They stated that the majority of the valve power loss is due to transitional throttling losses since with their design the number of transition events is doubled, but that those losses are offset by the self-spinning capability of the valve. Their testing showed that at $15 \mathrm{~Hz}$ the valve was more efficient than a similar bleed off circuit, and that if it were optimized it would be capable of between $77 \%$ and $84 \%$ efficient at $50 \%$ displacement. Transitional throttling losses were decreased because of the fast switching speed of the valve, but at $75 \mathrm{~Hz}$ it was found that the faster switching speeds cause the compressibility losses to negate other efficiency improvements. This finding makes it clear once again that throttling and compressibility losses need to be addressed, and that control valve design may not be the most effective solution.

In the study of the feasibility of PWM variable displacement pumps Rannow et al. state that power losses due to transitional throttling and compressibility are expected to be linear with high speed valve frequency, and that transitional throttling losses are predicted to be $72 \%$ of the energy loss per cycle [10]. In seeking solutions to the problem of transitional throttling loss in PWM circuits, Rannow and Li determined that generally $60 \%$ of system losses are due to valve transitions, $20 \%$ is due to fluid compressibility, $19 \%$ is due to throttling when the valve is fully open, and $1 \%$ is due to valve leakage [30]. 


\subsubsection{Soft Switching}

With the decrease in response time of electronic components, there has been an increased focus on the concept of soft switching in PWM circuits. A basic electronic soft switch inserts capacitance elements $\left(\mathrm{C}_{\mathrm{s}}\right)$ to absorb current when the transistor changes state [31]. The implementation of this soft switch approach in a DC-DC boost converter can be seen in Fig. 1.5. When these components are tuned properly, the overall effect is a reduction in losses due to transistor switches.

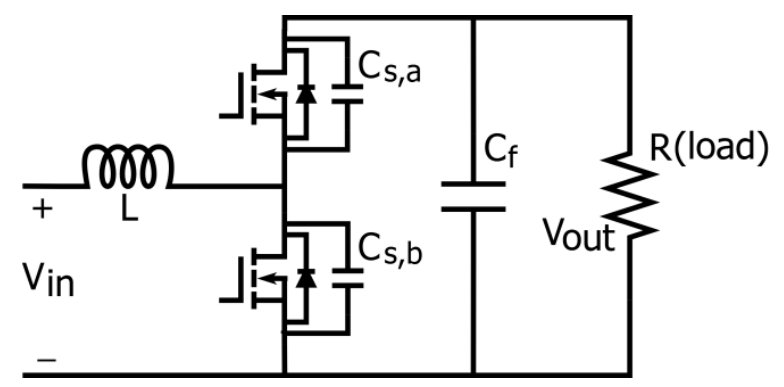

Figure 1.5 - DC-DC boost converter with soft switch capacitance elements included.

As demonstrated by initial switch-mode circuit analysis, the efficiency of a hydraulic system utilizing switch-mode techniques would be increased if throttling and compressibility losses were removed from the system. Research aimed at creating the hydraulic analog of electronic boost converters has focused on using passive capacitance elements such as accumulators to fill the role of capacitor. The hydraulic soft switching concept proposed by Rannow and $\mathrm{Li}$, which is based on electric soft switching, would remove more of the transitional and compressibility losses in PWM circuits, making VVDPs more efficient [30].

The soft switch concept, seen in Fig. 1.6, is a method whereby fluid that would normally be throttled across a control valve during a valve transition, instead flows into a small chamber with a spring-loaded piston (capacitance), to be released later after the valve transition is completed. Consider a PWM cycle where the cycle begins with the tank valve in the fully open position and the load valve in the fully closed position. First the tank valve will begin to close and the pressure will rise above the soft switch spring 
pressure, causing fluid to flow into the soft switch chamber. As the tank valve reaches a mostly closed position, the soft switch piston has displaced to a position near the middle of the chamber, where an externally active locking mechanism holds it in place. The pressure rises quickly opening the check valve, while simultaneously the tank valve has closed completely and the load valve transitions to the fully open position where the check valve will close and fluid will flow through the fully open load valve. After a short period of time the load valve closes, and as the tank valve begins to open the locking mechanism is released allowing fluid to flow into the soft switch chamber instead of being throttled across a partially open valve. Once the tank valve is fully open, the soft switch spring forces the piston back to zero displacement and the circuit is ready for another cycle.

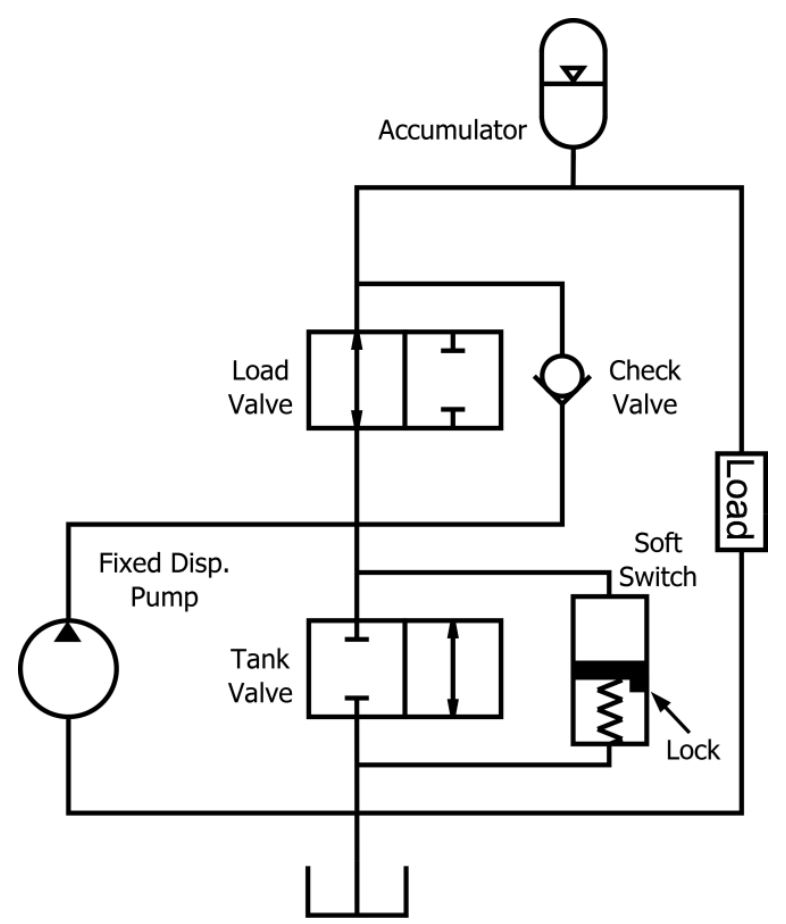

Figure 1.6 - Soft switch concept as proposed by Rannow and Li. During load and tank valve transitions, high pressure from the pump avoids being throttled by being absorbed by the soft switch. Adapted from [30].

Through numerical studies, Rannow and Li found that the soft switch approach can theoretically reduce throttling and compressibility losses by $81 \%$ and combined 
system losses by $64 \%$ when operating at $20 \mathrm{~Hz}$. Alternatively, the use of a soft switch that is 4.4 times slower than a baseline PWM circuit yields the same circuit efficiency [30]. The single soft switch proposed by Rannow and Li has two phases, the first phase is when it displaces to the lock point while the tank valve closes, and the second phase is when the lock releases during the opening of the tank valve. The main difficulty of the soft switch approach is the design of the soft switch lock release mechanism, which has to operate quickly during the right part of the PWM cycle. Though an exact method is not prescribed, piezo-electric actuators, pressure triggered latches, and internal on/off valve mechanisms are suggested as possible candidates for the unlocking mechanism [30].

The two phases of the soft switch can be divided into two soft switches, a passive switch and a locking switch, which allows for the study of a lock release mechanism separate from the passive element. To that end, Van de Ven proposed a lock and release mechanism for a soft switch [32]. This locking soft switch is a piston cylinder device with the back side of the piston ported across a check valve to load and the front side ported to the volume between the pump and control valve (Fig. 1.7). A force imbalance during the pressure control valve transition allows the piston to retract, opening the chamber to absorb fluid. In order for the unlocking mechanism to work properly, the check valve in parallel with the pressure valve is instead moved to a series position. The soft switch then absorbs flow during both control valve transition events. The soft switching circuit architectures shown and modeled by Van de Ven have an energy loss reduction in the range of $49.0 \%$ to $66.1 \%$ compared to a relief valve managing system pressure spikes. These values and system operation are theoretical, so experimental analysis is required to validate the claims of functionality. 


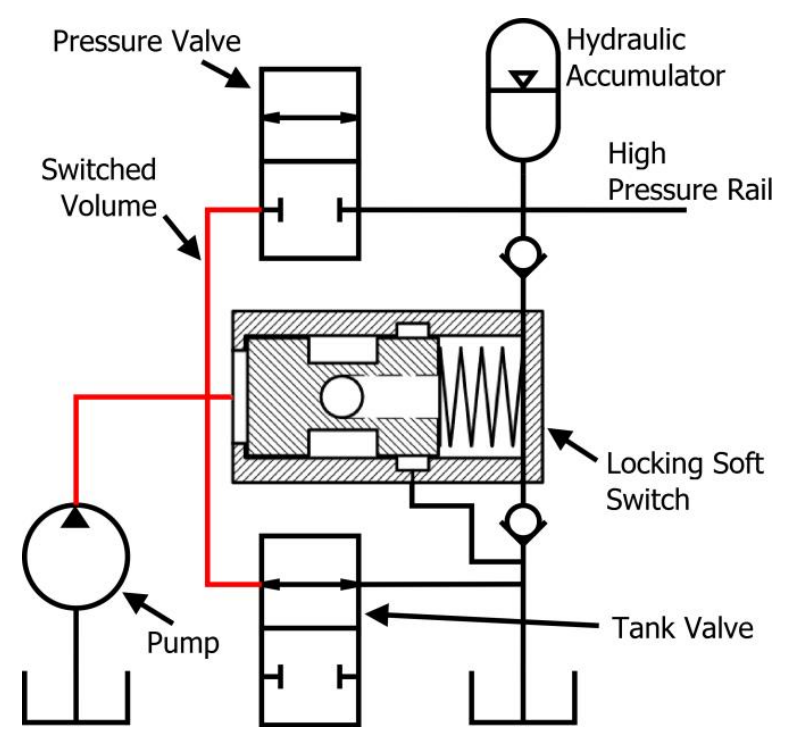

Figure 1.7 - Diagram of the virtually variable displacement pump locking soft switch circuit proposed by Van de Ven. Adapted from [32].

A numerical model of the PWM circuit utilizing the described soft switch was created by Triana, based on models presented by Van de Ven [32-34]. An optimization was performed with the end result being a theoretically efficient and easy to manufacture soft switch with a reduction in system losses of $70 \%$. A prototype was designed and built which failed to show proof of concept. It was observed that the soft switch would unlock prematurely causing it to be ineffective at absorbing fluid at the desired time. Triana suggests five main considerations to be included in further design work: include a method to bleed air out of the system before operation, design a method of adjusting the piston displacement necessary to unlock the soft switch, decrease leakage by extend the length of leakage paths around the soft switch piston, ensure proper sealing of check valves behind the soft switch, and design flow paths to be independent to allow easier troubleshooting [33]. The soft switch piston used by Triana also experienced significant wear due to material incompatibility. A redesign of the soft switch was commenced by the author to address the above design flaws as well as others. 


\subsection{Overview}

The research presented in this thesis builds on and extends the work done by Van de Ven and Triana at the University of Minnesota on the soft switch unlocking mechanism design and analysis [32,33]. The research focuses on: 1) creating a more robust numerical model of a PWM circuit with soft switching lock release, 2) the design, manufacturing, and assembly of the soft switch, and 3) tuning and testing the soft switch for experimental validation. The following chapters of this thesis describe this modeling, design, and experimentation in detail. Chapter 2 first describes the operation of all of the components in the soft switch circuit. The equations used to describe system pressures, flow rates, open areas, and accelerations are described along with their sources and limitations. Chapter 3 describes design changes for the soft switch and circuit, based on the numerical model described in Chapter 2 as well as limitations and errors found in the first prototype [33]. The system layout, electrical control circuit, and data acquisition systems for experimental testing are also outlined. Chapter 4 shows successful operation of the soft switch and describes its range of functionality. Further changes to the parameters in the numerical model are outlined that brought the numerical and physical systems into agreement. A comparison is then made between the efficiencies of throttling valve control, PWM without soft switches, and the VVDP utilizing the lock release soft switch. Chapter 5 concludes the thesis by summarizing the methods used, results obtained, and recommending future work for the soft switch lock release specifically and the soft switching concept generally. 


\section{Chapter 2:}

\section{Numerical Model}

In this chapter a numerical model of a virtually variable displacement pumping circuit with a soft switch is described. The model was created to provide a better understanding of the circuit and serve as a design tool for further optimization and implementation. The model used is based on work done by Van de Ven [32] in the initial concept generation phase and a prototype built and tested by Triana [33]. To aid in the understanding of the circuit behavior, the basic features of the soft switch and its normal operation are described. The equations used to describe system pressures, port areas, flow rates, and component positions are described, including justification of each equation. Circuit component specifications, and when necessary dimensions, will be shown and their use in the model will be explained. After a working model is achieved, it is used as a design guide for dimensioning a working prototype.

\subsection{Soft Switch Concept}

The soft switch lock release concept proposed by Van de Ven is shown in Fig. 2.1 [32]. It is a small piston cylinder device with the piston forced to the left end of the cylinder via a compression spring on the right side of the piston. There in an annulus in 
the middle of the piston that is internally ported to the right side of the piston. As the piston displaces to the right, the piston annulus aligns with an annulus in the cylinder that is ported to tank.

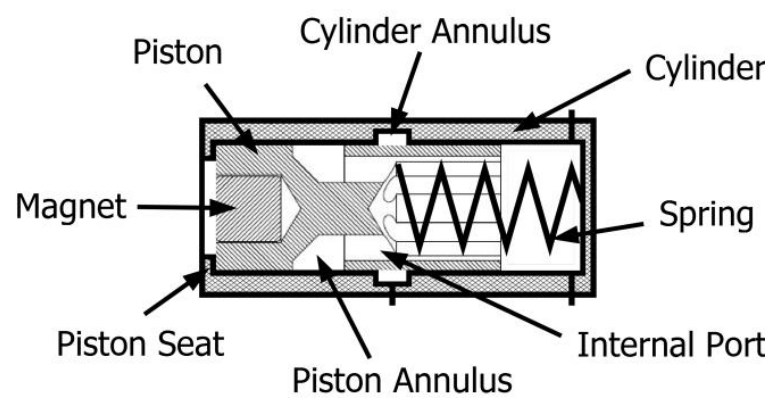

Figure 2.1 - Soft switch concept as proposed by Van de Ven. Adapted from [32].

Fig. 2.2 shows the layout of the virtually variable displacement pumping circuit which utilizes a fixed displacement pump and a high speed 3-way valve to test the functionality of the soft switch lock-release mechanism. The left side or front side of the soft switch cylinder is connected to the switched volume of the system, the cylinder annulus is ported to tank, and the right or back side of the cylinder is ported to the load and tank lines via check valves. 


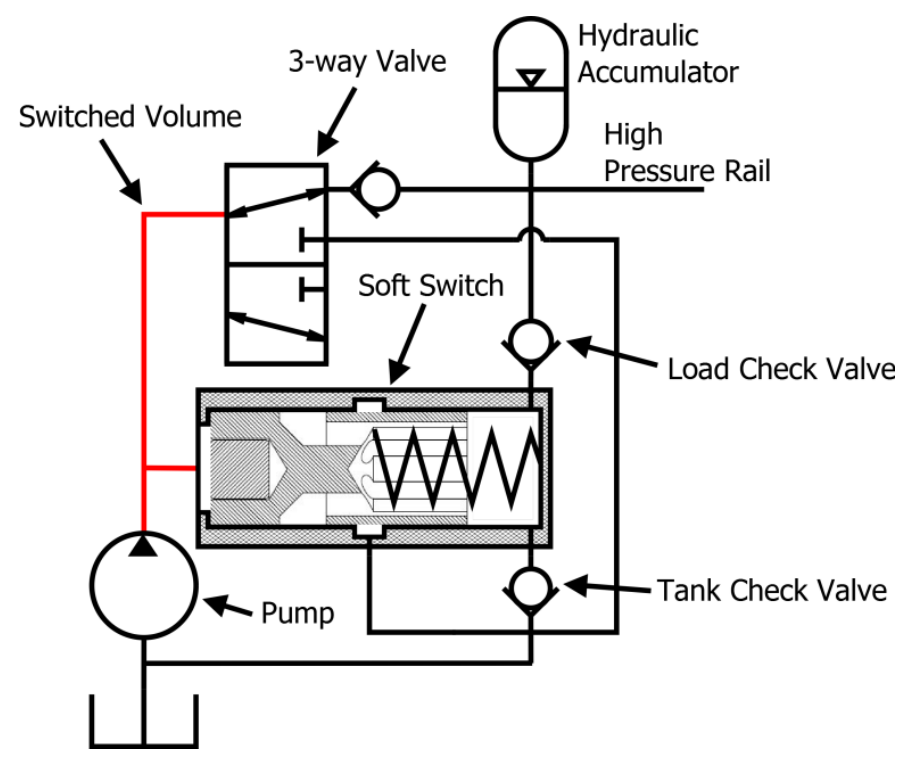

Figure 2.2 - Virtually variable displacement pumping circuit.

The behavior of the soft switch during one cycle of high speed valve PWM is as follows. The cycle begins with the high speed valve sending fluid to tank and the soft switch empty with the piston seated to the left. When the 3-way valve is transitioning from tank to load, the pressure in the switched volume increases as a result of throttling across the valve. This increased pressure moves the soft switch piston slightly to the right, compressing the spring and the fluid on the right of the piston. When the 3 -way valve finishes the transition to load, the piston remains stationary, except for slight movement due to leakage around the piston. As the 3-way valve pressure port begins to close, the pressure in the switched volume becomes greater than that of the load line, which forces the pressure check valve to open and the soft switch piston to displace even further to the right. When the piston has displaced enough for the annulus of the piston to align with the annulus of the cylinder, the piston is unlocked due to the decrease in pressure on the back side of the piston. The soft switch then rapidly absorbs the pump flow at tank pressure, which minimizes the throttling losses that the system would otherwise experience as the 3-way valve transitions from load to tank. Once the 3-way valve has switched completely back to tank, the spring on the back of the piston forces it 
back to its original position, sending the fluid inside the soft switch to tank, while simultaneously drawing fluid from tank into the back side of the soft switch. With the piston reseated, the switching cycle can begin again.

The rest of this chapter will describe the equations used to create a model of the soft switch operation. Justification for the equations will be given, including limitations to the model. Key measurements of circuit components will be introduced and applied to the equations.

\subsection{Model Equations}

This section outlines the equations used throughout the numerical model of the soft switch. Diagrams are provided when a visual description of components and equations enhances understanding.

\subsubsection{Port Positions and Component Areas}

The high speed control valve used in the model is an off-the-shelf 3-way valve made by Hydroforce (SV08-30). The port areas and shapes were determined by inspection. It was discovered that the ports used for the tank line are circular, whereas the load line ports are rectangular. When the valve spool is fully transitioned to tank, less than half of the area of the circular port is open. A graphical representation of spool displacement relative to tank and load port openings can be seen in Fig. 2.3, with the white moving plane representing spool displacement that allows the ports to open. While the ports in this figure are shown as single orifices, in the physical valve, there are multiple equally spaced ports along the radius of the valve spool and manifold. 
Load Port Tank Port

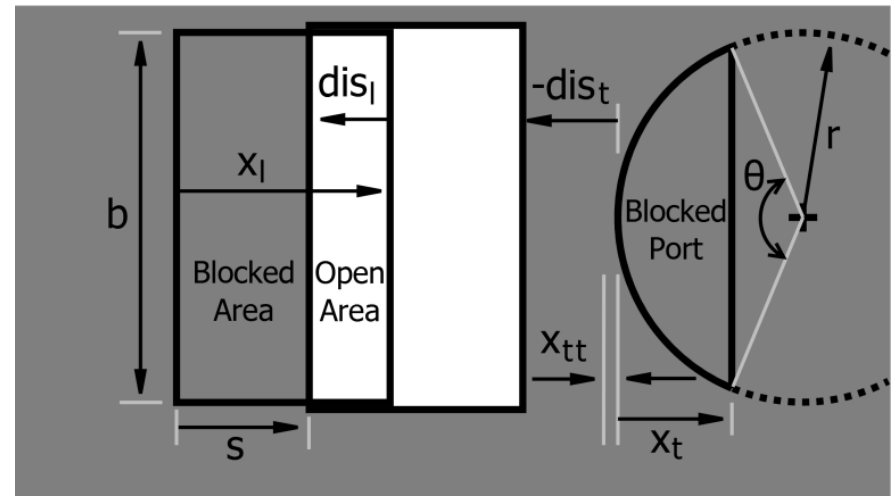

Figure 2.3 - Graphical representation of high speed valve spool displacement relative to tank and load port openings.

Since published valve information is limited, it is assumed that the valve spool displaces with a constant velocity, achieving the published valve transition times of 12 $\mathrm{ms}$ for the load port and $22 \mathrm{~ms}$ for the tank port. The maximum axial displacement of the valve spool was determined by measuring the axial lengths of the load and tank ports, as well as the length of the valve overlap where neither load nor tank ports are open.

$$
x_{\max }=x_{t}+x_{l}+x_{t t}
$$

where $x_{t}$ and $x_{l}$ are the lengths of the tank and load port openings along the spool axis respectively, $x_{t t}$ represents the length of the valve overlap where neither tank or load ports are open, and $x_{\max }$ is the maximum displacement of the valve spool.

Using Fig. 2.3 as a reference, the axial position of the valve spool, $s$, for time, $t$, is expressed in discretized form as:

$$
\left\{\begin{array}{llll}
\text { if } t \bmod T<t_{\text {off }} & s=s_{-1}-\frac{x_{\max }}{t t_{l}} \Delta t & \text { if } s \leq 0 & s=0 \\
\text { else } & s=s_{-1}+\frac{x_{\max }}{t t_{t}} \Delta t & \text { if } s \geq x_{\max } & s=x_{\max }
\end{array}\right.
$$

where $T$ is the switching period, defined as $1 /$ frequency, $t_{o f f}$ is the point in time at which the command signal is sent for the valve to be in the load position, defined as $T^{*} d u t y$, the -1 subscript denotes a value at a previous time-step, $t t_{l}$ and $t t_{t}$ are the transition 
times for the valve to move from tank to load position, and vice versa (respectively), and $\Delta t$ is the time step. The modulo function allows for evaluation of time relative to the switching period. The second layer of evaluations ensures that physical spool limits are enforced.

The specification sheet of the commercial valves contains plots of the pressure drop versus flow rate for fully open valve ports. The orifice equation:

$$
Q=C_{d} A \sqrt{\frac{2}{\rho} \Delta P}
$$

where $Q$ is flow rate, $C_{d}$ is the discharge coefficient, $A$ is the area of the port, $\rho$ is the mass density of hydraulic fluid, and $\Delta P$ is the pressure drop across the port, can be rearranged, and use data points from the plots to determine a lumped $C_{d} * A$ value. This method simplifies the model by ignoring the impact of geometry and flow conditions on the discharge coefficient.

By equating the lumped $C_{d} * A$ values calculated from Eq. 2.3, to a standard discharge coefficient value and the area formula for each port, an effective radius of the circular tank port and effective base of the rectangular load port can be calculated. This approach ensures accurate correlation between the model and published valve data.

The effective axial position of the open area of the moving plane relative to right edge of the load port, $x_{d i s, l}$, and the left edge of the tank port, $x_{d i s, t}$, is described by

$$
\begin{array}{lll}
x_{d i s, l}=x_{l}-s & \text { if } x_{d i s, l}<0 & x_{d i s, l}=0 \\
x_{d i s, t}=\left(s-x_{l}-x_{t t}\right) \frac{r_{t}(1-\cos \theta)}{x_{t}} & \text { if } x_{d i s, t}<0 \quad x_{d i s, t}=0
\end{array}
$$

where $r_{t}$ is the effective radius of the tank port and $\theta$ is the interior angle of the open circular tank segment. This calculation of effective spool position is required so that flow rate calculations will more accurately align with published valve parameters and properties.

Using the results of Eqs. 2.3 and 2.4, the orifice areas of the load and tank ports can be described by 


$$
\begin{gathered}
A_{l}=b_{l} x_{d i s, l} \\
A_{t}=r_{t}{ }^{2} \cos ^{-1}\left(1-\frac{x_{d i s, t}}{r_{t}}\right)+\left(\sqrt{\left(2 r_{t}-x_{d i s, t}\right) x_{d i s, t}}\left(x_{d i s, t}-r_{t}\right)\right)
\end{gathered}
$$

where $A_{l}$ is the effective orifice area for the load port, $A_{t}$ is the effective orifice area for the tank port, and $b_{l}$ is the effective length of the base of the load port.

The load and tank check valves seen in Fig. 2.2 are small disc style valves. The same lumped parameter calculations were performed in order to determine appropriate $C_{d} * A$ values. The maximum displacement of the check valve discs were measured, and then effective diameters of the check valve discs were determined using the calculated lumped parameter values. Assuming that the open areas for the check valves were a cylindrical surface area created by the displacement of the check valve disks, the open areas shown in blue in Fig. 2.4, can be represented mathematically as seen in Eq. 2.7.

$$
A_{c h, j}=\pi d_{c h, j} x_{c h, j}
$$

where $d_{c h, j}$ is the effective diameter of the check valve disk, $x_{c h, j}$ is the disk displacement, and the subscripts $l$ and $t$ are substituted into $y$ for load and tank respectively. This approach does not fully capture the relationship between check valve disk displacement and open area, since there is a small lip in the check valve body next to the disk seat as shown in Fig. 2.4. Considering the lip is only $2.5 \%$ of the maximum displacement of the check valve disk, the effects were considered to be negligible. 


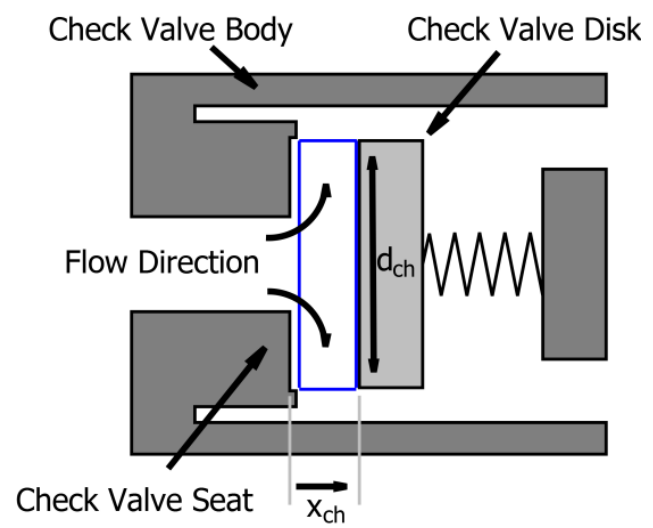

Figure 2.4 - Open area of check valves corresponds to the blue cylindrical surface area between the check valve disk and seat.

Perhaps the most difficult port area to describe and quantify is the piston port to tank shown in Fig. 2.5. The port on the piston itself is an annulus, while the port on the cylinder initially opens to a set of holes that are equally spaced around the cylinder surface, before flow moves into an annulus around the exterior of the cylinder and finally through a port to tank (Fig. 2.1). Initially when the soft switch piston displaces and the port to tank opens, only a section of the circular ports is open, followed by the circular ports fully opening. Depending on the piston displacement limits during testing, the piston could displace to the point where the ports start becoming blocked again. This behavior is described by the following pseudo-code

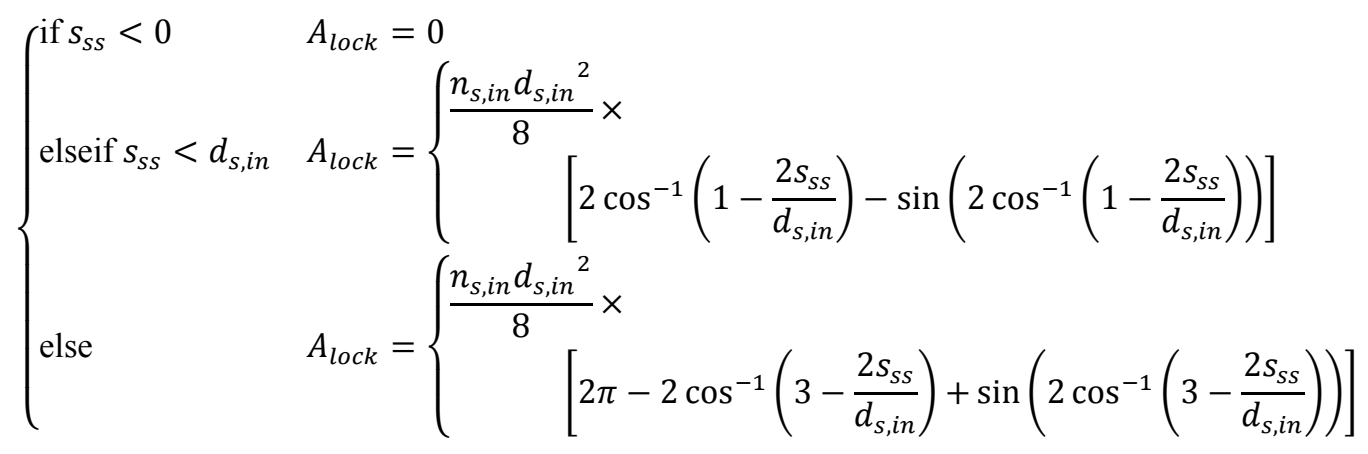

where $S_{S S}$ is the soft switch piston displacement referenced at the point where the edge of the piston annulus and cylinder port first align and begin to open to flow, $A_{\text {lock }}$ is the area of the piston port to tank, $d_{s, i n}$ is the diameter of the cylinder port holes, and $n_{s, \text { in }}$ is the number of cylinder port holes. 


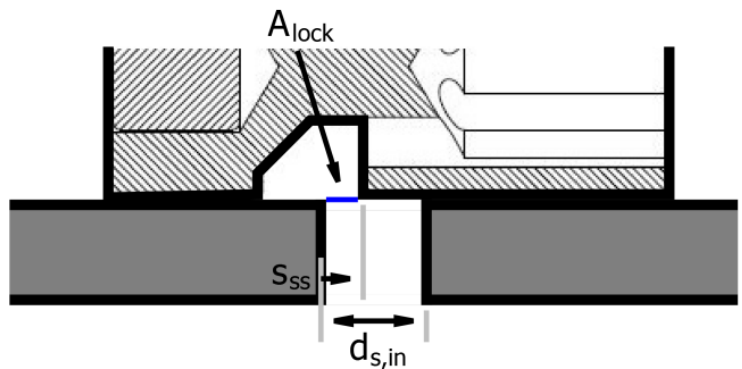

Figure 2.5 - Open area of the soft switch internal port to tank. The port is comprised of 6 identical holes through the cylinder sleeve.

The parameters used to calculate port positions and component areas are summarized in Table 2.1. The results of Eqs. $2.2-2.6$ can be seen in the plots of the high speed valve command signal, displacement, and valve port orifice areas for a duty ratio of $60 \%$ shown in Fig. 2.6. 
Table 2.1 - The parameters required to calculate the port positions and open areas of all of the soft switch circuit components.

\begin{tabular}{|c|c|c|c|}
\hline Parameter & Symbol & Units & Value \\
\hline \multicolumn{4}{|l|}{ System parameters: } \\
\hline Switching period & $T$ & $\mathrm{~s}$ & 0.15 \\
\hline Duty ratio & duty & unitless & 0.6 \\
\hline Time-step & $\Delta t$ & $\mathrm{~s}$ & $1 e-6$ \\
\hline \multicolumn{4}{|l|}{ 3-way Valve Parameters: } \\
\hline Height of the load port & $x_{l}$ & $\mu \mathrm{m}$ & 660 \\
\hline Height of the tank port & $x_{t}$ & $\mu \mathrm{m}$ & 787 \\
\hline Gap between tank and load ports & $x_{t t}$ & $\mu \mathrm{m}$ & 127 \\
\hline Angle of max. open circular tank & $\theta$ & $\mathrm{rad}$ & 2.36 \\
\hline Discharge Coeff*Area for load port & $C_{d} A_{l}$ & $\mathrm{~mm}^{2}$ & 6.35 \\
\hline Discharge Coeff*Area for tank port & $C_{d} A_{t}$ & $\mathrm{~mm}^{2}$ & 5.98 \\
\hline Effective base of rectangular load port & $b_{l}$ & $\mathrm{~mm}$ & 16.0 \\
\hline Effective radius of the tank port & $r_{t}$ & $\mathrm{~mm}$ & 3.47 \\
\hline Load transition time & $t t_{l}$ & $\mathrm{~ms}$ & 12 \\
\hline Tank transition time & $t t_{t}$ & $\mathrm{~ms}$ & 22 \\
\hline \multicolumn{4}{|l|}{ Soft Switch Parameters: } \\
\hline Diameter of sleeve tank port holes & $d_{s, i n}$ & $\mathrm{~mm}$ & 5.08 \\
\hline Number of cylinder port holes & $n_{s, i n}$ & unitless & 6 \\
\hline \multicolumn{4}{|l|}{ Check Valve Parameters: } \\
\hline Discharge Coeff*Area for load C.V. & $C_{d} A_{c h, l}$ & $\mathrm{~mm}^{2}$ & 4.50 \\
\hline Discharge Coeff*Area for tank C.V. & $C_{d} A_{c h, t}$ & $\mathrm{~mm}^{2}$ & 14.1 \\
\hline Effective diameter of load C.V. disc & $d_{c h, l}$ & $\mathrm{~mm}$ & 3.09 \\
\hline Effective diameter of tank C.V. disc & $d_{c h, t}$ & $\mathrm{~mm}$ & 5.47 \\
\hline
\end{tabular}



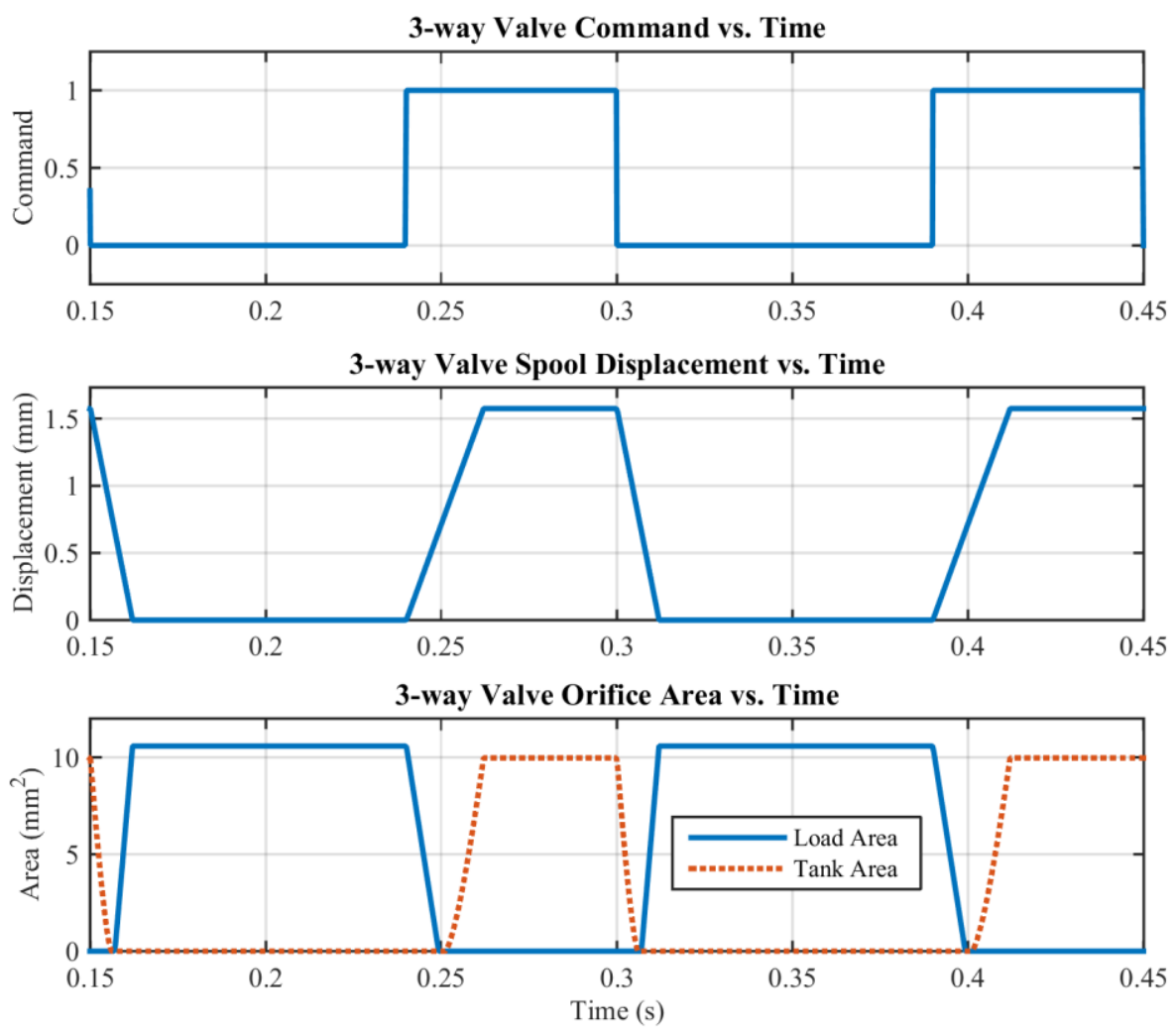

Figure 2.6 - High speed valve command signal, spool displacement, and orifice areas as a function of time. Two switching periods are shown, at a duty ratio of $60 \%$.

\subsubsection{Pressures}

There are four pressures of interest in the VVDP circuit shown in Fig. 2.7; load line pressure, tank pressure, switched volume pressure, and pressure behind the soft switch. The load line is assumed to remain at a constant pressure due to the presence of the accumulator on that line. The tank also remains constant but at atmospheric pressure. The pressures in the switched volume and behind the soft switch are more complex to model due to soft switch dynamics. Since the hydraulic fluid is experiencing rapid pressurization and depressurization at large magnitudes it is appropriate to consider the fluid compressible. The bulk modulus of a fluid is a measure of the compressibility of the fluid, therefore both of these pressures are derived from the definition of the tangent bulk modulus [1] 


$$
\beta=-V \frac{d P}{d V}
$$

where $\beta$ is the tangent bulk modulus of air free oil, $d V$ is change in volume of the fluid of interest, and $d P$ is the change in pressure. The bulk modulus of hydraulic oil is a strong function of pressure and has a small temperature dependence. In this work, the temperature dependence will be neglected, while the pressure dependence will be captured by calculating an effective bulk modulus.

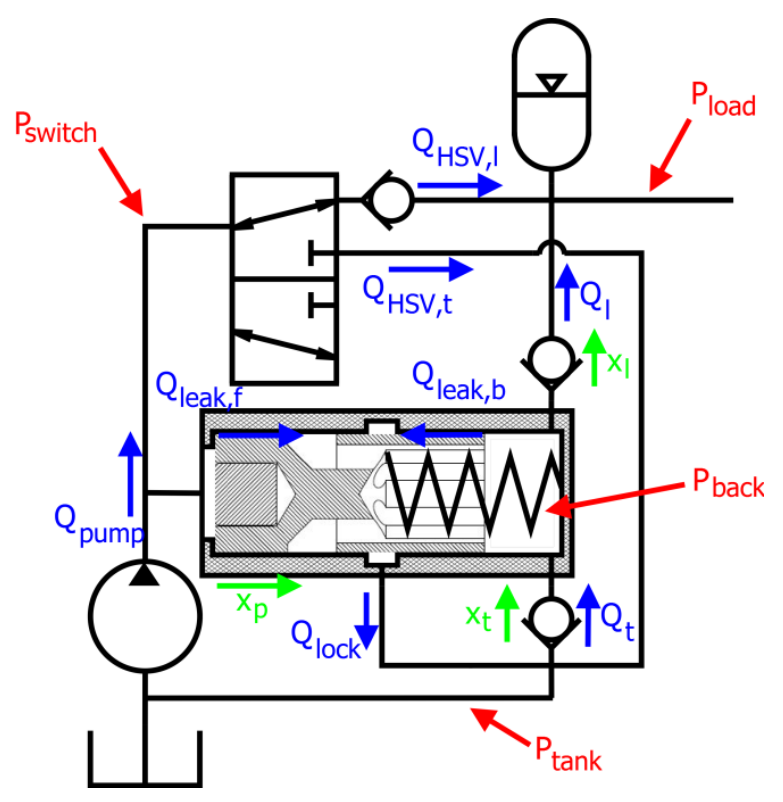

Figure 2.7 - Pressures, flow rates, and displacements of interest.

Gholizadeh et al. analyzed multiple bulk modulus modeling approaches [35], determining that the Cho et al. model offers adequate accuracy with a fairly simple implementation [36]. The Cho model takes into account air that is entrained in the hydraulic fluid, assumes that the total air content and the solubility of air are both constant, assumes that the system is operating under low pressure, neglects the mass of air mixed in the fluid, and assumes that fluid compression and decompression are adiabatic. This effective bulk modulus is shown in Eq. 2.10 


$$
\beta_{e}(P)=\beta\left[\frac{\left(\frac{P}{P_{0}}\right)^{\frac{1}{\gamma}} e^{\frac{\left(P_{0}-P\right)}{\beta}}+R}{\frac{R}{\gamma} \frac{\beta}{P}+\left(\frac{P}{P_{0}}\right)^{\frac{1}{\gamma}} e^{\frac{\left(P_{0}-P\right)}{\beta}}}\right]
$$

where $\beta_{e}(P)$ is the effective bulk modulus, $P_{0}$ is atmospheric pressure, $\gamma$ is the ratio of specific heats for air, and $R$ is the volume fraction of air entrained in the oil.

An expression for pressure in a fluid volume can be found by rearranging the definition of flow rate

$$
Q=\frac{d V}{d t}
$$

where $d t$ is a change in time. Eqs. 2.9 and 2.11 can be combined, yielding an equation that represents the change in pressure of a volume of interest. The pressure in the switched volume (shown in Fig. 2.7) is described as

$$
\begin{aligned}
& \dot{P}_{\text {switch }}=\frac{\beta_{e}\left(P_{\text {switch }}\right)}{\left(A_{p} x_{p}+V_{\text {switch }}\right)} \times \\
& \left(Q_{\text {pump }}-Q_{H S V, l}-Q_{H S V, t}-Q_{\text {leak,f }}-A_{p} v_{p}\right)
\end{aligned}
$$

where the volume of interest is composed of the volume of fluid from the pump to the cylinder, $V_{\text {switch }}$ and the cylinder volume due to piston displacement, $Q_{\text {pump }}$ is the flow rate out of the pump, $Q_{H S V, l}$ and $Q_{H S V, t}$ are the flow rates through the 3-way valve to load and tank respectively, $Q_{\text {leak,f }}$ is the leakage flow rate from the switched volume to the volume behind the soft switch, and $x_{p}$ is the displacement of the piston.

The pressure behind the soft switch piston can also be found with this approach:

$$
\begin{gathered}
\dot{P}_{\text {back }}=\frac{\beta_{e}\left(P_{\text {back }}\right)}{\left(V_{\text {dead }}+V_{\text {back }}\right)} \times \\
\left(Q_{t}+Q_{\text {leak }, f}-Q_{l}-Q_{\text {lock }}-Q_{\text {leak }, b}+A_{p} v_{p}\right)
\end{gathered}
$$

where $V_{\text {dead }}$ is the dead volume behind the piston, $V_{\text {back }}$ is the changing volume behind the piston (neglecting dead volume), $Q_{t}$ and $Q_{l}$ are the flow rates through the tank and load check valves respectively, $Q_{\text {lock }}$ is the flow rate through the locking port, and $Q_{\text {leak,b}}$ is the leakage flow rate from the back of the soft switch to tank. 
Notice that in both Eqs. 2.12 and 2.13, leakage past the soft switch piston is considered, but leakage flow past the high speed valve is considered to be negligible and is therefore not included. The parameters used to calculate system pressures are summarized in Table 2.2.

Table 2.2 - The parameters required to calculate the system pressures.

\begin{tabular}{lccc}
\hline \hline Parameter & Symbol & Units & Value \\
\hline System parameters: & \multicolumn{1}{c}{} & $\mathrm{s}$ & $1 \mathrm{e}-6$ \\
Time-step & $\Delta t$ & $\%$ & 2 \\
Entrained air in switched volume & $R_{\text {switch }}$ & $\%$ & 2 \\
Entrained air in volume behind piston & $R_{\text {back }}$ & $\%$ & 1.9 \\
Tangent bulk modulus of air free oil & $\beta$ & $\mathrm{GPa}$ & 1.4 \\
Ratio of specific heats for air & $\gamma$ & unitless & 101 \\
Atmospheric pressure & $P_{0}$ & $\mathrm{kPa}$ & 10.786 \\
Pump flow rate & $Q_{\text {pump }}$ & liters $/ \mathrm{min}$ & 3.786 \\
Switched volume & $V_{\text {switch }}$ & $\mathrm{cm}^{3}$ & 30 \\
Dead volume behind piston & $V_{\text {dead }}$ & $\mathrm{cm}^{2}$ & 1.87 \\
Soft Switch Parameters: & \multicolumn{3}{|c}{} \\
Area of piston face & $A_{p}$ & $\mathrm{~mm}^{2}$ & 127 \\
\hline \hline
\end{tabular}

\subsubsection{Flow Rates}

Assuming that the speed of the prime mover is constant and that there is no leakage past the pump, the flow rate from the pump is considered constant. The flow through the valves throughout the circuit is modeled using the orifice equation (Eq. 2.3), which carries with it the limitations of the Bernoulli equation [37]. The Bernoulli equation assumes steady flow, negligible viscous effects and heat transfer, no shaft work, that the flow is incompressible, and that all of the flow follows the same streamline. The discharge coefficient seen in the orifice equation lumps all of the losses and steady flow limitations of this equation into one parameter that is found through valve testing.

The flow rate through the high speed valve tank port is described below by the orifice equation with the variable area calculated in Eq. 2.6 


$$
Q_{H S V, t}=C_{d} A_{t} \sqrt{\frac{2}{\rho}\left|P_{\text {switch }}-P_{\text {tank }}\right|} \operatorname{sgn}\left(P_{\text {switch }}-P_{\text {tank }}\right)
$$

where $P_{\text {tank }}$ is the pressure of the hydraulic reservoir.

The circuit shown in Fig. 2.7 utilizes a check valve in series with the high speed valve load port to ensure that flow reversals do not occur in the load line. For ease of simulation, the effects of the load port and the check valve on flow rate are combined. Since the pressure drop across the two components is additive, the orifice equation is solved for pressure and the terms describing the two pressures are added together, utilizing the variable load port area calculated in Eq. 2.5

$$
P_{\text {switch }}-P_{\text {load }}-P_{l l}=\frac{\rho Q_{H S V, l}}{2}\left[\frac{1}{\left(C_{d} A_{l}\right)^{2}}+\frac{1}{\left(C_{d} A_{c h, l l}\right)^{2}}\right]
$$

where $P_{l o a d}$ is the constant load pressure, $P_{l l}$ is the cracking pressure of the load line check valve, and $C_{d} A_{c h, l l}$ is the lumped discharge coefficient and area for the load line check valve, based on the check valve specification sheet. Eq. 2.15 is then solved for the combined flow rate of the valves

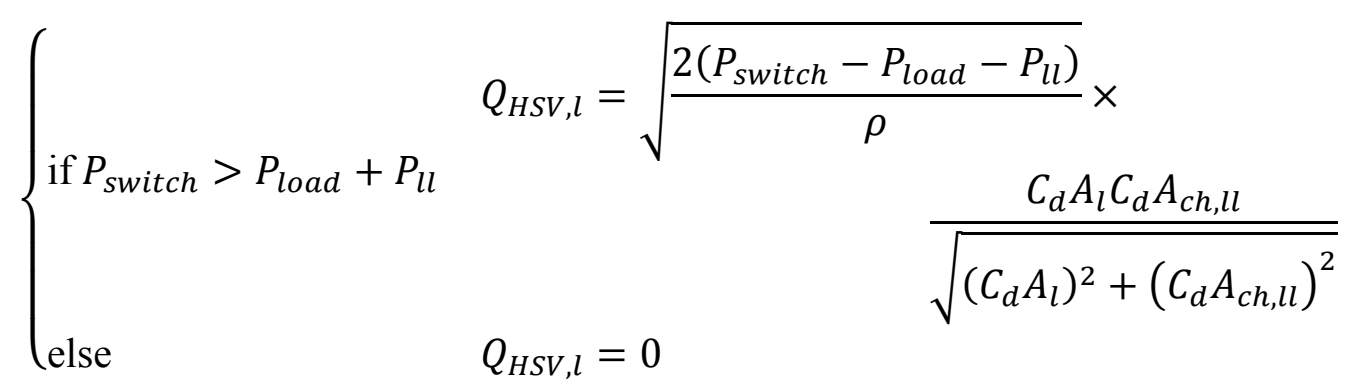

In this case the two equations are needed because it is assumed that the check valve closes instantaneously when the load line pressure increases above the combined switched and load line cracking pressures, effectively eliminating the possibility of backflow.

Two other check valves are used to connect the volume behind the soft switch piston to the tank and load lines. Modeling their behavior is done differently than the load line check valve in Eq. 2.16, since the dynamics of these valves have the potential to be much more influential on the operation of the soft switch. Like the high speed valve 
ports, the flow through the check valves is modeled using the orifice equation and the variable areas calculated previously (Eq. 2.7)

$$
\begin{aligned}
& Q_{l}=C_{d} A_{c h, l}\left(x_{c h, l}\right) \sqrt{\frac{2\left|P_{\text {back }}-P_{\text {load }}-P_{l}\right|}{\rho}} \operatorname{sgn}\left(P_{\text {back }}-P_{\text {load }}-P_{l}\right) \\
& Q_{t}=C_{d} A_{c h, t}\left(x_{c h, t}\right) \sqrt{\frac{2\left|P_{\text {tank }}-P_{\text {back }}-P_{t}\right|}{\rho}} \operatorname{sgn}\left(P_{\text {tank }}-P_{\text {back }}-P_{t}\right)
\end{aligned}
$$

where $P_{l}$ and $P_{t}$ are the cracking pressures of the load and tank check valves respectively.

The last port flow rate of interest in the soft switch circuit is through the soft switch locking port. One side of the port is connected to the back of the soft switch piston, while the other is connected to the tank, and the flow rate through it is described by the following conditional equation

$$
Q_{\text {lock }}=C_{d} A_{\text {lock }} \sqrt{\frac{2}{\rho}\left|P_{\text {back }}-P_{\text {tank }}\right|} \operatorname{sgn}\left(P_{\text {back }}-P_{\text {tank }}\right)
$$

where the conditional statement allows for backflow.

As the soft switch is not designed as a positive seal device, leakage flow occurs between the piston and accompanying cylinder. Assuming no slip boundary conditions, that the working fluid is incompressible and Newtonian, and that the leakage flow is laminar, the Navier-Stokes equation [37] can be solved to describe leakage between parallel plates

$$
Q_{\text {leak }}=\frac{2 b h^{3} \Delta P}{3 \mu L}
$$

where $b$ is the width of the flow, $h$ is half of the gap between the plates, $\Delta P$ is the change in pressure from one end of the leakage path to the other, $\mu$ is the dynamic viscosity of the hydraulic fluid, and $L$ is the axial length of the leakage path. Since the clearance between the soft switch piston and associated cylinder is small relative to the diameter of the piston, the curvature of the piston has little affect, and the equation remains accurate. 
The leakage flow paths past the piston are shown in Fig. 2.8. One of the paths, shown in blue, is leakage from the switched volume at the front of the piston to the volume behind the soft switch piston via the piston annulus, while the other leakage path, shown in green, is from the volume at the back of the soft switch to tank via both the piston annulus and the right side of the piston.

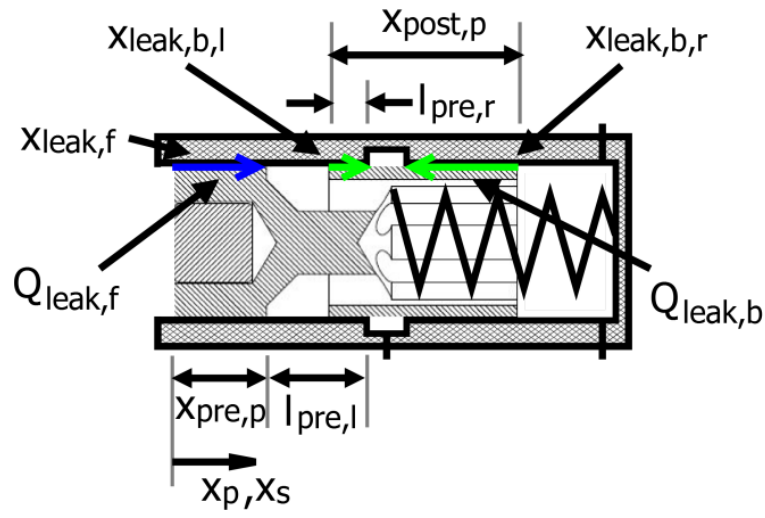

Figure 2.8 - Leakage flow paths past the soft switch piston. The path shown in blue goes from the switched volume at the front of the piston to the volume behind the piston. The path shown in green travels from the back of the soft switch to tank via two paths, as a result of the internal porting of the piston.

Due to the movement of the piston, the lengths of the leakage paths are always changing. A mathematical description of the leakage path lengths is given in Eqs. 2.21 and 2.22. Note that to facilitate the testing of the soft switch lock release mechanism, the piston cylinder was designed as a sleeve within a manifold, which allowed the piston to displace further to the left than the cylinder sleeve. This sleeve design, as well as the multiple leakage paths from the back of the soft switch to tank, increases the complexity of the leakage length calculations as can be seen below.

$$
\begin{cases}\text { if } x_{p}<x_{s} & x_{\text {leak }, f}=x_{\text {pre }, p}-x_{s}+x_{p} \\ \text { elseif } x_{p}<l_{\text {pre }, l}+x_{s} & x_{\text {leak }, f}=x_{\text {pre }, p} \\ \text { else } & x_{\text {leak }, f}=x_{\text {pre }, p}+l_{\text {pre }, l}+x_{s}-x_{p}\end{cases}
$$




$$
\begin{gathered}
\begin{cases}\text { if } x_{p}<\frac{x_{\text {post }, p}}{2}+x_{s} & x_{\text {leak }, b, r}=\frac{x_{\text {post }, p}}{2}-x_{s}+x_{p} \\
\text { else } & x_{\text {leak }, b, r}=x_{\text {post }, p}\end{cases} \\
x_{\text {leak }, b, l}=l_{\text {pre }, r}+x_{s}-x_{p}
\end{gathered}
$$

where $x_{s}$ is the displacement given to the sleeve relative to the left of the manifold, $x_{l e a k, f}$ is the axial length of the leakage path at the front of the piston, $x_{p r e, p}$ is the axial length of the left end of the piston, $l_{p r e, l}$ is the distance (relative to zero displacement of the sleeve) that the piston has to travel in order to begin closing the port to tank, $x_{\text {post,p }}$ is the axial length of the right end of the piston, $x_{l e a k, b, r}$ is the axial length of the leakage path at the back of the piston, on the right side, $x_{l e a k, b, l}$ is the axial length of the leakage path at the back of the piston, on the left side, and $l_{p r e, r}$ is the distance (relative to zero displacement of the sleeve) that the piston has to travel in order to open the port to tank. The combination of the sleeve displacement, $x_{s}$, and piston travel to open port to tank, $l_{\text {pre,r }}$, constitutes the piston unlocking distance, $x_{\text {unlock }}$.

The leakage from the front of the piston is quantified using Eq. 2.20 and the leakage length from the front of the piston to the piston annulus (Eq. 2.21):

$$
Q_{\text {leak }, f}=\frac{2 \pi d_{p} h_{p}{ }^{3}\left(P_{\text {switch }}-P_{\text {back }}\right)}{3 \mu x_{\text {leak }, f}}
$$

where $d_{p}$ is the diameter of the piston, and $h_{p}$ is half of the radial clearance between the piston and the cylinder. The leakage from the back of the piston to the internal tank port is quantified similarly, using the leakage lengths from the back of the piston to the tank port (Eqs. 2.22):

$$
\begin{cases}\text { if } x_{p}<l_{\text {pre }, r}+x_{s} & Q_{\text {leak }, b}=\frac{2 \pi d_{p} h_{p}{ }^{3}\left(P_{\text {back }}-P_{\text {tank }}\right)}{3 \mu x_{\text {leak }, b, r} x_{\text {leak }, b, l}} \times \\ \text { else } & Q_{\text {leak }, b}=\frac{2 \pi d_{p} h_{p}{ }^{3}\left(P_{\text {back }}-P_{\text {tank }}\right)}{3 \mu x_{\text {leak }, b, r}}\end{cases}
$$


Table 2.3 summarizes the parameters that are needed to calculate the flow rates through each circuit component.

Table 2.3 - The parameters required to calculate the flow rates through each circuit component, as well as leakage flow rates.

\begin{tabular}{|c|c|c|c|}
\hline Parameter & Symbol & Units & Value \\
\hline \multicolumn{4}{|l|}{ System parameters: } \\
\hline Time-step & $\Delta t$ & $\mathrm{~s}$ & $1 e-6$ \\
\hline Generic discharge coefficient & $C_{d}$ & unitless & 0.6 \\
\hline Mass density of oil & $\rho$ & $\mathrm{kg} / \mathrm{m}^{3}$ & 876 \\
\hline Load pressure & $P_{\text {load }}$ & $\mathrm{MPa}$ & 7 \\
\hline Tank pressure & $P_{\text {tank }}$ & $\mathrm{kPa}$ & 101 \\
\hline \multicolumn{4}{|l|}{ 3-way Valve Parameters: } \\
\hline Discharge Coeff*Area for load line C.V. & $C_{d} A_{c h, l l}$ & $\mathrm{~mm}^{2}$ & 7.375 \\
\hline Cracking pressure for load line C.V. & $P_{l l}$ & $\mathrm{kPa}$ & 103 \\
\hline \multicolumn{4}{|l|}{ Soft Switch Parameters: } \\
\hline Piston diameter & $d_{p}$ & $\mathrm{~mm}$ & 12.7 \\
\hline Length of piston before tank annulus & $x_{\text {pre }, p}$ & $\mathrm{~mm}$ & 7.62 \\
\hline Length of piston after tank annulus & $x_{\text {post }, p}$ & $\mathrm{~mm}$ & 15.2 \\
\hline Length of piston tank annulus & $A_{\text {lock }}$ & $\mathrm{mm}$ & 5.08 \\
\hline Piston/cylinder gap & $c_{p}$ & $\mu \mathrm{m}$ & 12.7 \\
\hline Piston displacement to open tank port & $l_{\text {pre,r }}$ & $\mathrm{mm}$ & 2.54 \\
\hline Piston displacement to close tank port & $l_{\text {pre }, l}$ & $\mathrm{~mm}$ & 7.62 \\
\hline Initial sleeve displacement & $x_{s}$ & $\mathrm{~mm}$ & 0 \\
\hline \multicolumn{4}{|l|}{ Check Valve Parameters: } \\
\hline Cracking pressure of load C.V. & $P_{l}$ & $\mathrm{kPa}$ & 103 \\
\hline Cracking pressure of tank C.V. & $P_{t}$ & $\mathrm{kPa}$ & 20.7 \\
\hline
\end{tabular}

\subsubsection{Component Positions}

The last things that need to be calculated are the positions of circuit components, namely the soft switch piston and check valve disks. The acceleration of the soft switch piston is determined by doing a force balance on the piston (Eq. 2.25), using Newton's second law of motion. The piston experiences movement due to the pressures on either side of the piston, the preload force of the spring behind the piston, the compression of the spring due to piston displacement, and the viscous friction related to the movement of the piston. The viscous friction term assumes laminar Couette flow between flat plates 
[37]. The velocity and displacement of the piston are then determined by numerically integrating the acceleration term.

$$
\begin{gathered}
\ddot{x}_{p}=\frac{\left(P_{\text {switch }}-P_{\text {back }}\right) A_{p}-k_{s} x_{p}-F_{s}-\frac{\mu \dot{x}_{p} A_{p, \text { surf }}}{c_{p}}}{m_{p}} \\
\dot{x}_{p}=\int \ddot{x}_{p} d t \\
x_{p}=\int \dot{x}_{p} d t
\end{gathered}
$$

where $A_{p}$ is area of the piston face, $k_{s}$ is the spring constant for the spring behind the piston, $F_{S}$ is the force on the piston due to the spring preload, $A_{p, \text { surf }}$ is the surface area of the piston exposed to the cylinder surface, $c_{p}$ is the clearance between the piston and the cylinder, and $m_{p}$ is the mass of the piston.

The physical limits of the soft switch piston are enforced by conditional statements on the displacement and velocity terms:

$$
\begin{gathered}
\begin{cases}\text { if } x_{p}<0 & x_{p}=0 \\
\text { elseif } x_{p}>x_{p, \max } & x_{p}=x_{p, \max }\end{cases} \\
\begin{cases}\text { if } \dot{x}_{p}<0 \text { and } x_{p} \leq 0 & \dot{x}_{p}=0 \\
\text { elseif } \dot{x}_{p}>0 \text { and } x_{p} \geq x_{p, \max } & \dot{x}_{p}=0\end{cases}
\end{gathered}
$$

where $x_{p, \max }$ is the maximum displacement of the soft switch piston. This approach does not take into account the possibility of the piston bouncing upon impact with the zero displacement limit, but the chance of bounce occurring will be very small as a result of the preload force of the spring behind the piston. This assumption was confirmed experimentally, as described in Chapter 4.

The check valve disk accelerations, velocities, and displacements are calculated using the same force balance approach used on the soft switch piston. 


$$
\begin{gathered}
\ddot{x}_{t}=\frac{\left(P_{\text {tank }}-P_{\text {back }}\right) A_{d, t}-k_{t} x_{t}-P_{t} A_{d, t}-\frac{\mu \dot{x}_{t} A_{t, \text { surf }}}{c_{t}}}{m_{t}} \\
\dot{x}_{t}=\int \ddot{x}_{t} d t \\
x_{t}=\int \dot{x}_{t} d t \\
\ddot{x}_{l} \frac{\left(P_{\text {back }}-P_{\text {load }}\right) A_{d, l}-k_{l} x_{l}-P_{l} A_{d, l}-\frac{\mu \dot{x}_{l} A_{l, \text { surf }}}{c_{l}}}{m_{l}} \\
\dot{x}_{l}=\int \ddot{x}_{l} d t \\
x_{l}=\int \dot{x}_{l} d t
\end{gathered}
$$

where $A_{d}$ is the area of the disc face, $k$ is the spring rate, $x$ is the displacement of the disc, $P$ is the cracking pressure, $A_{\text {surf }}$ is the surface area of the disc exposed to the valve body, $c$ is the clearance between the disc and the body, $m$ is the mass of the check valve disc, and the subscripts $l$ and $t$ denote load and tank check valves respectively.

The travel and velocity limits of the check valve discs are enforced as follows:

$$
\begin{gathered}
\begin{cases}\text { if } x_{j}<0 & x_{j}=0 \\
\text { elseif } x_{j}>x_{j, \max } & x_{j}=x_{j, \text { max }}\end{cases} \\
\left\{\begin{array}{l}
\dot{x}_{j}=0 \\
\text { elseif } \dot{x}_{j}<0 \text { and } x_{j} \leq 0
\end{array} \quad \dot{x}_{j}=0\right.
\end{gathered}
$$

where $l$ and $t$ are substituted for $j$ to represent load and tank respectively, and $x_{t, \text { max }}$ and $x_{l, \max }$ are the maximum displacements of the tank and load check valve discs respectively. As with the modeling of the soft switch piston, this conditional statement approach does not take into account the possibility of the check valve discs bouncing upon impact with their physical limits. The check valve spring preload force is much 
smaller than that of the piston, so there is a possibility that using the conditional statements will not adequately describe the seating of the check valves, which would cause the model to underestimate the fluid flow through a similar physical system.

The parameters used to calculate the displacements of the soft switch and check valve discs are summarized in Table 2.4.

Table 2.4 - The parameters required to calculate the accelerations, velocities, and displacements of the soft switch and check valve discs.

\begin{tabular}{|c|c|c|c|}
\hline Parameter & Symbol & Units & Value \\
\hline \multicolumn{4}{|l|}{ System parameters: } \\
\hline Time-step & $\Delta t$ & $\mathrm{~s}$ & $1 \mathrm{e}-6$ \\
\hline Dynamic viscosity of oil & $\mu$ & $\mathrm{Pa}^{*} \mathrm{~s}$ & 0.0404 \\
\hline \multicolumn{4}{|l|}{ Soft Switch Parameters: } \\
\hline Area of piston face & $A_{p}$ & $\mathrm{~mm}^{2}$ & 127 \\
\hline Surface area of piston & $A_{p, \text { surf }}$ & $\mathrm{mm}^{2}$ & 790 \\
\hline Piston mass & $m_{p}$ & $\mathrm{~g}$ & 17.0 \\
\hline Piston/cylinder clearance & $c_{p}$ & $\mu \mathrm{m}$ & 12.7 \\
\hline Spring preload force & $F_{S}$ & $\mathrm{~N}$ & 22.2 \\
\hline Spring rate & $k_{s}$ & $\mathrm{kN} / \mathrm{m}$ & 5 \\
\hline Maximum piston displacement & $x_{p, \max }$ & $\mathrm{mm}$ & 12.7 \\
\hline \multicolumn{4}{|l|}{ Check Valve Parameters: } \\
\hline Actual area of load C.V. face & $A_{d, l}$ & $\mathrm{~mm}^{2}$ & 26.1 \\
\hline Actual area of tank C.V. face & $A_{d, t}$ & $\mathrm{~mm}^{2}$ & 48.7 \\
\hline Surface area of load C.V. disc & $A_{l, \text { surf }}$ & $\mathrm{mm}^{2}$ & 13.8 \\
\hline Surface area of tank C.V. disc & $A_{t, \text { surf }}$ & $\mathrm{mm}^{2}$ & 32.7 \\
\hline Mass of load C.V. & $m_{l}$ & $\mathrm{mg}$ & 155 \\
\hline Mass of tank C.V. & $m_{t}$ & $\mathrm{mg}$ & 502 \\
\hline Clearance of load C.V. & $c_{l}$ & $\mathrm{~mm}$ & 1.44 \\
\hline Clearance of tank C.V. & $c_{t}$ & $\mathrm{~mm}$ & 1.71 \\
\hline Cracking pressure of load C.V. & $P_{l}$ & $\mathrm{kPa}$ & 103 \\
\hline Cracking pressure of tank C.V. & $P_{t}$ & $\mathrm{kPa}$ & 20.7 \\
\hline Spring rate of load C.V. & $k_{l}$ & $\mathrm{~N} / \mathrm{m}$ & 225 \\
\hline Spring rate of tank C.V. & $k_{t}$ & $\mathrm{~N} / \mathrm{m}$ & 170 \\
\hline Maximum displacement of load C.V. & $x_{l, \max }$ & $\mathrm{mm}$ & 1.02 \\
\hline Maximum displacement of tank C.V. & $x_{t, \max }$ & $\mathrm{mm}$ & 1.52 \\
\hline
\end{tabular}




\subsection{Model Architecture and Functionality}

The differential equations in the model described in the previous section were solved using an Eulerian approach [38], which allowed for a simple implementation with reasonable accuracy (as long as the time-step is small enough). After initializing parameters, the model loop was solved in the following order: 3-way valve spool position; 3-way valve, internal piston, and check valve port areas; flow rates through the pump, 3-way valve, check valve, and internal piston ports, and leakage paths; piston and check valve states; bulk moduli and pressures in the switched volume and behind the soft switch.

A plot of the soft switch piston displacement with respect to time for one cycle of operation is shown in Fig. 2.9. Initially the soft switch is empty at zero displacement.

During the 3-way valve transition to load the switched volume pressure begins to rise and the soft switch begins to displace and fill, reducing the throttling across the partially open control valve. When the switched volume pressure grows larger than the load pressure fluid is able to flow through the check valve to load. While the control valve is open to load, the soft switch continues to displace slightly due to leakage past the piston. When the control valve transitions to tank, the switched volume pressure begins to rise again, displacing the soft switch piston to the unlocking location. Once this displacement is reached, the pressure behind the soft switch decreases rapidly, allowing the soft switch to absorb the pump flow, which in turn decreases the switched volume pressure. The piston is shown vibrating in an open position (notice that it never reaches the maximum displacement of $12.7 \mathrm{~mm}$ ) for a moment, before the 3-way valve completes the transition back to tank and the piston reseats. 

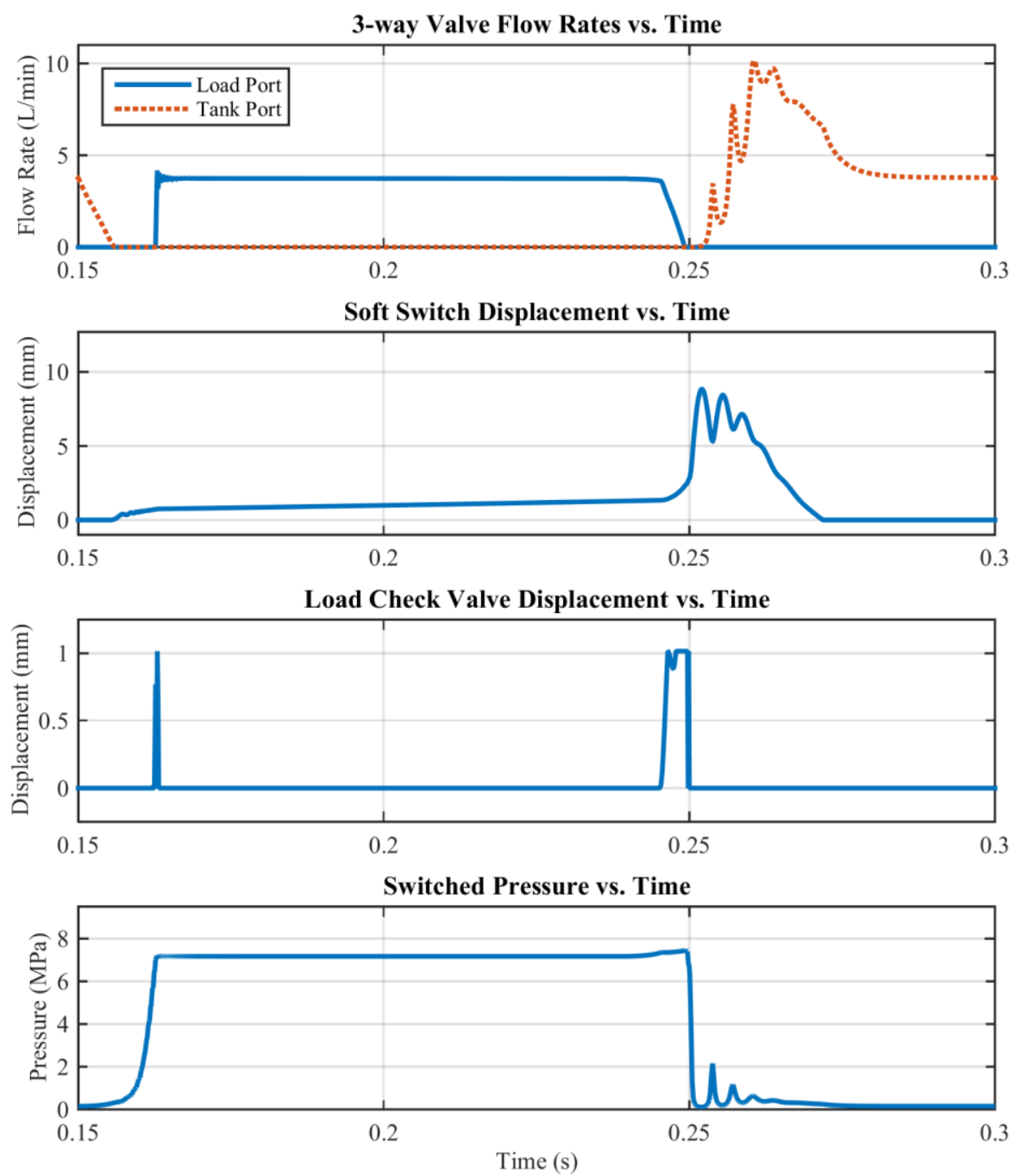

Figure 2.9 - Numerical model performance plots. As the 3-way valve transitions to load, the soft switch displaces due to switched volume pressure. The transition to tank causes the soft switch to unlock and absorb the pump flow that would normally be throttled across the control valve.

\subsection{Model Development Conclusion}

This chapter described a functional model of a virtually variable displacement pumping circuit. The system pressures of interest were modeled using an effective bulk modulus approach that takes into account bulk modulus pressure dependence. Port areas for the 3-way control valve are calculated using measurements of the port sizes and a 
lumped port area and discharge coefficient. System flow rates are given by the orifice equation with calculated system pressures. Leakage flow rates past the soft switch piston are calculated as laminar Couette flow. Soft switch piston and check valve disc positions are given by integrating force balances based on Newton's law of motion. The numerical model provides a design tool for further soft switch optimization and design. 


\section{Chapter 3:}

\section{Physical Model Development}

This chapter focuses on physical components and system design for both the initial prototype and the current build of the soft switch. First a description of the initial prototype and an explanation of the testing circuit layout for the first prototype will be given, along with findings by Triana [33]. Shortcomings and oversights to the initial prototype will be given, Triana's suggestions for improvement will be outlined, and the design of the new physical model will be given. The new test circuit will be shown, and preparations for testing on the circuit will be described. Finally, the control of the system components and data acquisition will outlined. Experimental results will be presented in Chapter 4.

\subsection{Physical Model Background}

Van de Ven showed that reducing the size of the switched volume (Fig. 2.2) had a favorable effect on the efficiency of the system by reducing compressibility losses [34]. As a result, the initial soft switch prototype, designed and built by Triana, was in a small manifold that mounted directly onto the body of the 3-way valve (Fig. 3.1). The 3-way valve body was also mounted as closely to the pump outlet as possible, again to reduce 
the switched volume. The check valves from the back side of the soft switch to load and tank were implemented in the soft switch manifold. The flow from the load check valve was internally ported to the 3-way valve manifold load port, and the flow from both the tank check valve and the unlocking port to tank were also internally ported together to reduce the complexity of the fluid lines leaving the soft switch manifold block. Included in the design was an ingenious piston travel limiter and spring preload adjuster combination, for soft switch parameter tuning. The manufacturing of the soft switch cylinder ports was simplified through use of a sleeve design. The optimized soft switch parameters can be seen in Table 3.1.

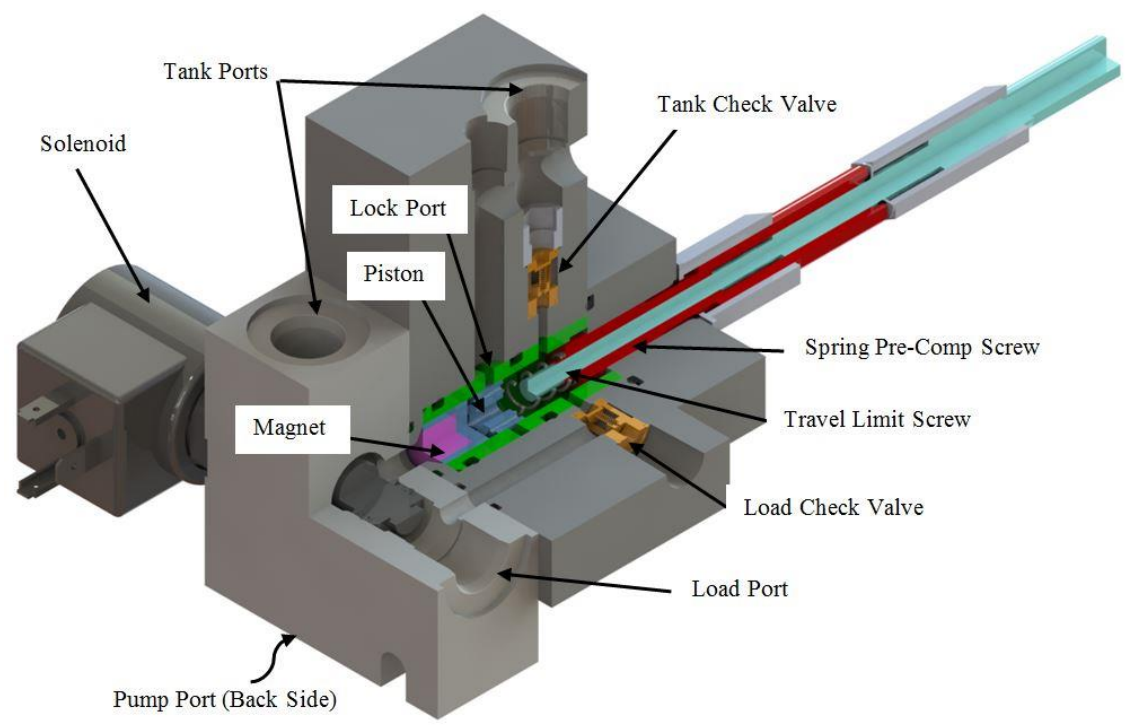

Figure 3.1 - Initial soft switch lock-release prototype. Used with permission [33]. 
Table 3.1 - Triana's optimized soft switch parameters after prescribing a soft switch radius that ensures ease of displacement measurement during testing [33].

\begin{tabular}{lcc}
\hline \hline Parameter & Units & Value \\
\hline Piston radius & $\mathrm{mm}$ & 6.35 \\
Piston length before crack & $\mathrm{mm}$ & 12.7 \\
Piston crack port length & $\mathrm{mm}$ & 2.5 \\
Piston length after crack & $\mathrm{mm}$ & 2.5 \\
Soft switch dia. clearance & $\mu \mathrm{m}$ & 35 \\
Maximum soft switch & $\mathrm{mm}$ & 6.5 \\
Soft switch preload & $\mathrm{N}$ & 18 \\
\hline \hline
\end{tabular}

The test circuit was organized as seen in Fig. 3.2, with three pressure transducers, two flow meters, a needle valve to provide the system load, and a displacement sensor for determining soft switch piston location. This arrangement allowed for measurements of switched volume pressure, pressure behind the soft switch, load pressure, tank flow rate, load flow rate, and piston position. From these measurements the efficiency of the system was calculated, and the timing of the soft switch piston could be understood and tuned to increase system efficiency. With the circuit completed and data acquisition tools prepared, testing began.

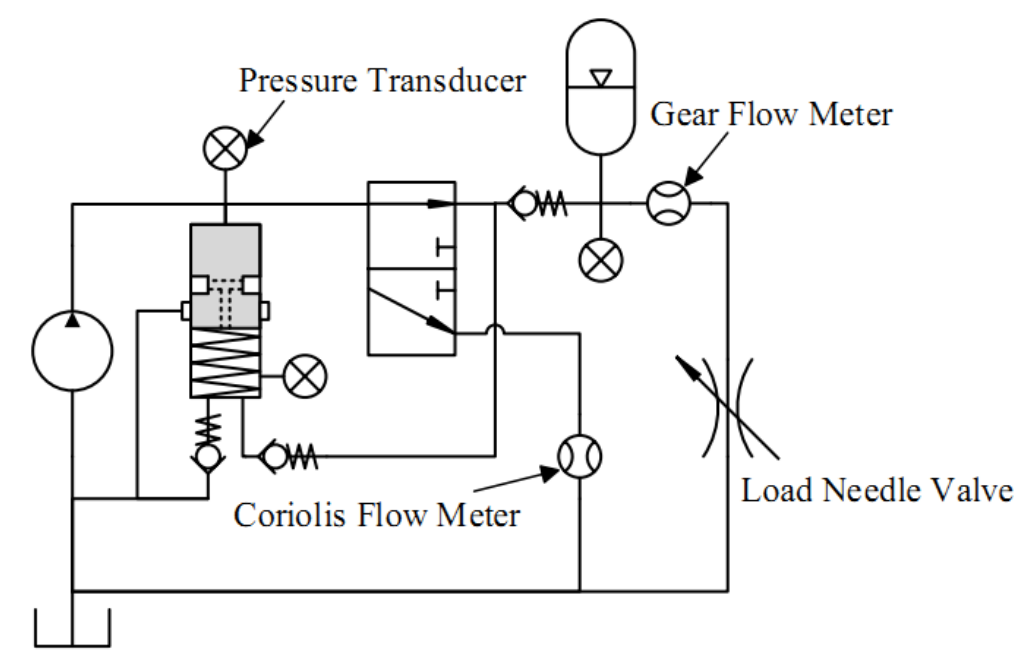

Figure 3.2 - Initial soft switch lock-release prototype circuit. Used with permission [33]. 
It was discovered that the soft switch was not behaving as expected. The soft switch piston displaced to its extreme position almost immediately, and it was assumed that this displacement was due to unanticipated manifold leakage as well as trapped air within the system. Attempts to bleed the air out of the manifold appeared to be unsuccessful, and as a result of the internal porting of the manifold, leakage paths could not be identified with any certainty. Triana suggested the following six design changes to consider for a second prototype [33]:

1) A system for simplified bleeding of the circuit

2) A method for tuning the piston displacement required for unlocking

3) Longer leakage paths along the piston walls

4) Redesign of check valves and check valve ports

5) Independent flow paths

6) Better position sensor mounting.

Reasons for each suggested design change and how the issues were resolved will be discussed hereafter.

\subsection{Prototype Redesign}

The redesigned soft switch, mounted to the 3-way control valve is seen in Fig. 3.3. The soft switch manifold has a main bore wherein the cylinder sleeve and piston are inserted. The piston is free to travel axially within the cylinder between the stop on the left and the displacement limiting screw on the right. A compression spring provides a preload force to the piston that returns it to the seated position at the left of the cylinder. The piston is designed with internal porting to allow fluid to travel to the piston annulus, which when aligned with the sleeve tank ports performs the soft switch unlocking function. The load and tank check valves are placed near the rear of the manifold and ported to the back of the piston through holes in the cylinder sleeve. Ports are provided at both ends of the manifold to allow for pressure transducer connection. 


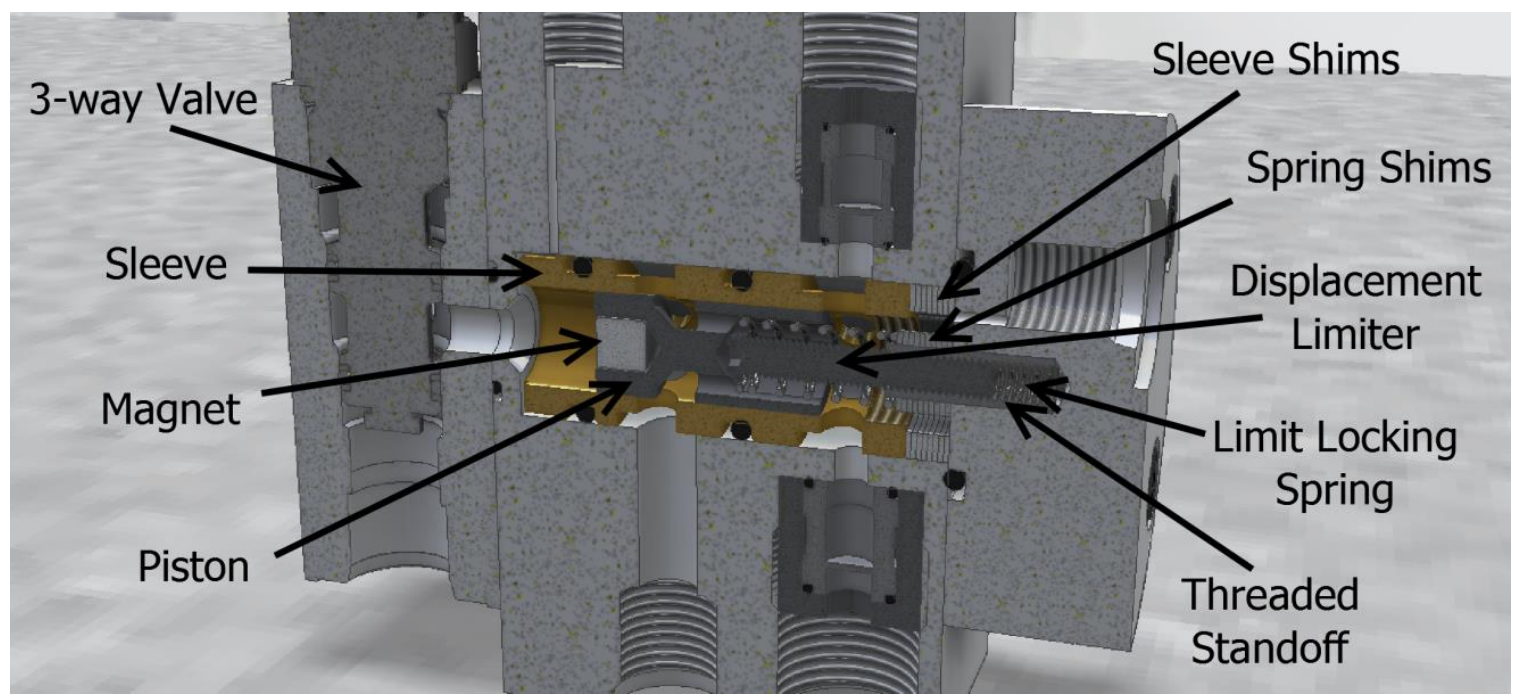

Figure 3.3-Cross-sectional view of the assembled soft switch, including 3-way valve and manifold.

\subsubsection{Piston and Sleeve Design}

The piston and sleeve were redesigned to address the issue of the piston unlocking early in the switching cycle. This was accomplished by changing the piston unlock position, checking the piston and sleeve clearance dimensions, and changing the component materials. These changes necessitated adjustments in the unlocking port and leakage path dimensions, in both the piston and sleeve, as well as adjustments to the preload spring seat on the piston.

With the soft switch piston unlocking prematurely in the first prototype, it was determined that it would be advantageous to control the distance that the piston is required to displace prior to unlocking. The model (Eqs. 2.23 and 2.24) indicates that the leakage past the piston is highly dependent on the clearance between the piston and sleeve, which is cubed in those equations. If the soft switch diametral clearance increased by twice the value of $35.6 \mu \mathrm{m}$ (measured off the Triana prototype), the leakage past the piston would allow for premature unlocking of the switch if the unlocking port only required $2.54 \mathrm{~mm}$ of piston displacement (which is the value that it was designed to). An easy way to allow for variability in the unlocking distance of the piston was to allow for shims to be placed at the front and back of the sleeve to change the location of the 
unlocking port to tank. It was determined that allowing the unlocking crack distance to vary a total of $5.08 \mathrm{~mm}$ in $0.635 \mathrm{~mm}$ increments, with the minimum unlocking distance being the $2.54 \mathrm{~mm}$ prescribed by Triana, would allow for far more variability than required.

As the position of the sleeve is adjustable, the port to tank on the sleeve needs to align with the manifold port to tank no matter the displacement of the sleeve. The sleeve was therefore designed with circular ports extending radially at the desired unlocking location, which then opened to a wide annular groove around its exterior (Fig. 3.4). The ports to the tank and load check valves at the back of the soft switch used the same circular port to annular groove design. An O-ring groove was placed on either side of the unlocking tank port to ensure that no leakage flow could occur between the sleeve and the manifold bore.

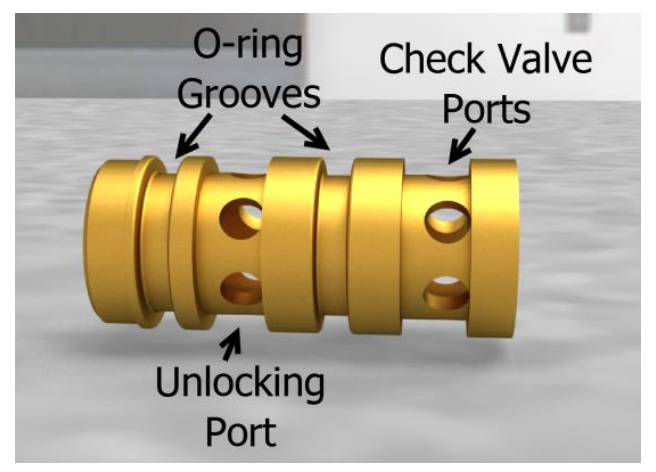

Figure 3.4 - The soft switch sleeve tank and load ports were made up of circular holes that open up to annuli. Two O-ring grooves allowed for positive seal against the soft switch manifold.

Designing the sleeve tank ports to be circular also helped to minimize another potential issue. The previous sleeve tank ports were designed as notches on either side of the internal surface, which may have promoted wear on the piston as it moved past the notches. The circular ports of the redesign allowed for a more guided movement of the piston, reducing wear. This design also proved useful in the case that the piston was outfitted with O-rings in an attempt to create a positive seal or to analyze unanticipated leakage paths (such as those experienced in the first prototype). The diameter of the port 
holes was chosen such that at all unlocked piston displacements, the internal port to tank was open, allowing for easier piston reseating.

The front of the sleeve was also designed with a notch and an annular groove that allowed for easy fluid flow during the bleeding of the front of the soft switch (Fig. 3.5). Also, the back end of the sleeve was threaded for removal of the sleeve from the manifold body during shim placement.

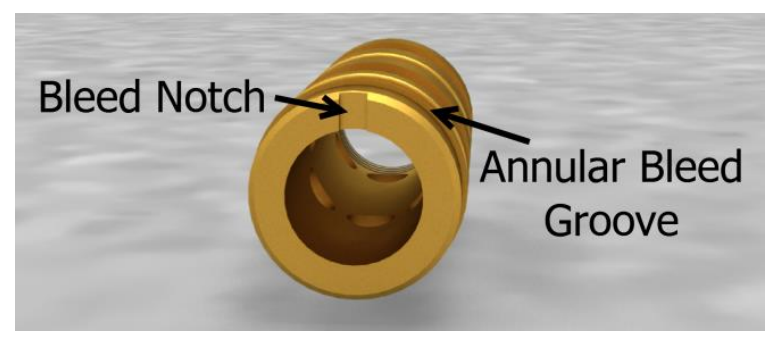

Figure 3.5 - The left end of the soft switch sleeve had a radial notch and an annular groove that allowed fluid to more easily flow around the front cavity of the soft switch, which promoted air release during system bleeding.

The original piston design utilized a $2.54 \mathrm{~mm}$ annular groove as the unlocking port, which limited the unlocking displacement of the piston by not allowing the port to be open for the entire displacement of the piston. Increasing the width of the groove to $5.08 \mathrm{~mm}$ allowed the port to be at least partially open for twice the distance the previous design allowed (Fig.3.6), which is important, since maximum piston displacement was another parameter that was determined should be variable. The model showed that under designed operating conditions, the maximum displacement of the piston was less than 10 $\mathrm{mm}$, as seen in Fig. 2.9. This increased groove width allowed for a piston displacement of $12.7 \mathrm{~mm}$. 


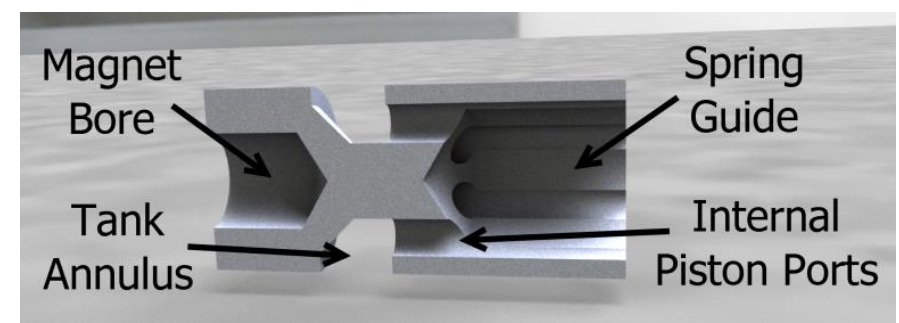

Figure 3.6 - Cross-sectional view of the soft switch piston. The bore on the left end was for the embedded magnet, while the large bore on the right side housed the left end of the compression spring.

The lengths of the both ends of the piston were also increased to allow for the new displacement ability and to increase the leakage path lengths between the piston and sleeve. In order to accommodate a spring with the correct spring rate and length for the desired preload force, the right side of the piston was bored out. The left end of the piston was bored out to create a space for a magnet to be embedded along the axis for use in linear displacement sensing. Holes were also drilled from the right side of the piston to the annulus to complete the piston internal unlocking port. The original piston used a main hole through the center of the piston for the internal tank flow. Many smaller holes were drilled around it to ensure that when the port was covered by the displacement limiter, reseating could still occur. The size of the compression spring used for the piston preload dictated that a smaller diameter displacement limiter be used, which made the use of a central hole for the internal port unrealistic. A circular hole pattern was decided upon, with six $2.54 \mathrm{~mm}$ diameter holes. A chart showing energy loss through the piston per switching cycle as a function of port area was created to determine if this arrangement would over constrict the flow through the port (Fig. 3.7). The model was run at a $60 \%$ duty ratio, and as the energy going to load for one cycle is about $36 \mathrm{~J}$, it can be seen that an energy loss of $1.5 \%$ is not too extreme. Since an increase in hole diameters would weaken the structure of the piston, it was determined that the design was adequate. 


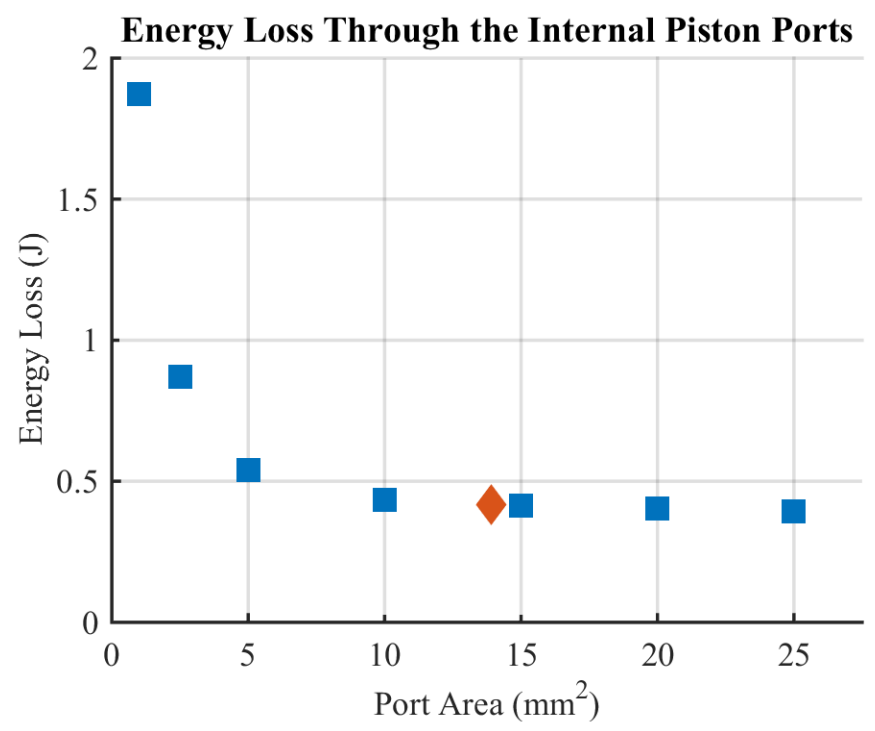

Figure 3.7 - Energy loss through internal piston ports for one switching cycle. The red marker indicates the energy loss through the designed port area.

The piston and sleeve were both manufactured out of stainless steel in the first prototype, in order to eliminate changes in the magnetic field given by the embedded magnet. This led to excessive wear of the piston and sleeve, possibly contributing to the leakage problem experienced during testing. In the redesign, the piston remained stainless steel, and the sleeve material was changed to bearing bronze. This combination of a soft metal against a hard metal reduced galling while still being non-ferrous. The detail design of the piston and sleeve can be seen in Figs. A.3 and A.4 of Appendix A.

\subsubsection{Spring, Limits, and Check Valves}

In the initial prototype design, an ingenious contraption was designed to allow for easy adjustment of the soft switch piston displacement limit and spring preload value. It was made up of a combination of bolts and nuts that were sealed via O-rings, and turned out to be leaky and difficult to adjust. Adjustment of the spring compression interfered with the travel limit location and vice versa. 
In the redesign, the preload spring was adjusted by adding shims to the spring seat area at the right end of the spring. Eqs. 2.25 - 2.27 in the model determine the position of the soft switch piston through integration of a force balance on the piston. With the pressures and piston sleeve clearance indicated in Table 2.3 it was determined that the soft switch unlocks at the appropriate time in the switching cycle for a preload force in the range of 15 to 40 Newtons. In the end a spring was found that, combined with the shims, would allow the preload force of the spring to be adjustable from 8.25 Newtons to 28.5 Newtons, giving a wide adjustment with over half of it being within the expected operational range. The travel limit was achieved using a simple screw as a backstop (Fig. 3.8). The screw was held in place by a spring inserted into the threaded hole, with the desired maximum and minimum displacements being achieved by either switching out the screw with a shorter or longer one or threading the screw in or out of the threaded hole. In order to simplify the manufacture of the plate holding the spring and limiter, a simple hole was drilled along the centerline and a threaded standoff was inserted via an interference fit. A small hole and threaded port were also drilled into the plate that allowed a pressure transducer to be attached to the outside. The detail design of the face plate can be seen in Fig. A.2 in Appendix A.

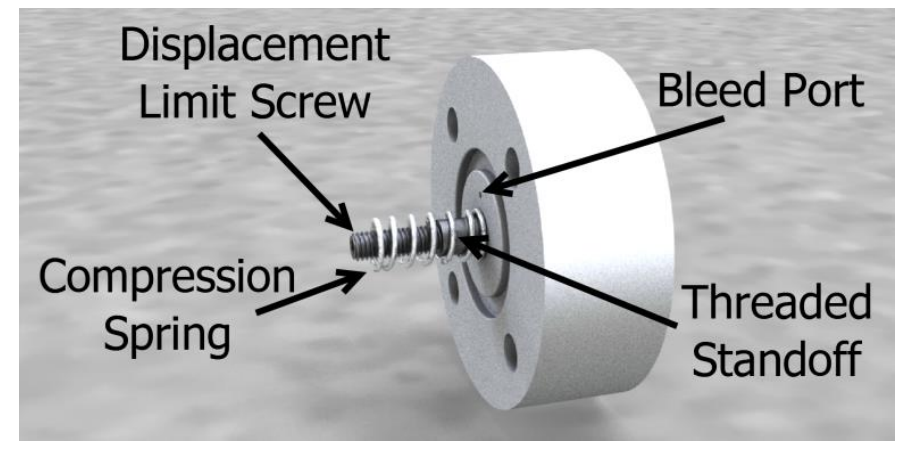

Figure 3.8 - The face plate of the soft switch manifold, including the piston displacement limiter and the compression spring behind the soft switch.

The check valves utilized in the initial prototype were small and designed to be inserted into small manifold cavities. Because of their small size they are extremely delicate and prone to deformation when exposed to excessive axial force during seating. 
Deformation of the check valves was evident in the disassembly of the first prototype and it is thought to be one of the unexpected leakage paths causing soft switch failure. It also appeared that the cracking pressure of the tank check valve may have been too high (possibly $103 \mathrm{kPa}$ ), causing cavitation when fluid needed to be drawn through it for soft switch reseating. To solve these problems, proper check valve housings were purchased that allowed for proper seating of the check valves without adding too much more to their size (Fig. 3.9). The housings threaded into place via a hexagonal shaped through hole. A tank check valve with a cracking pressure of $20.7 \mathrm{kPa}$ (the smallest that could be purchased in this design) and a larger nominal flow rate was used in order to reduce the possibility of cavitation when fluid was being drawn from tank to reseat the soft switch piston. As seen in Fig. 2.9, the pressure check valve is required to open at two different times in a switching cycle, each time a fast reseat with small backflow is desirable to reduce losses. A low cracking pressure is desired so that the pressure signal from the load line can be accurately felt, but in order to minimize the flow through the check valve while it is reseating, the cracking pressure should be increased. A cracking pressure of $103 \mathrm{kPa}$ was chosen to allow for low pressure drop while maintaining faster dynamics as suggested by the model.

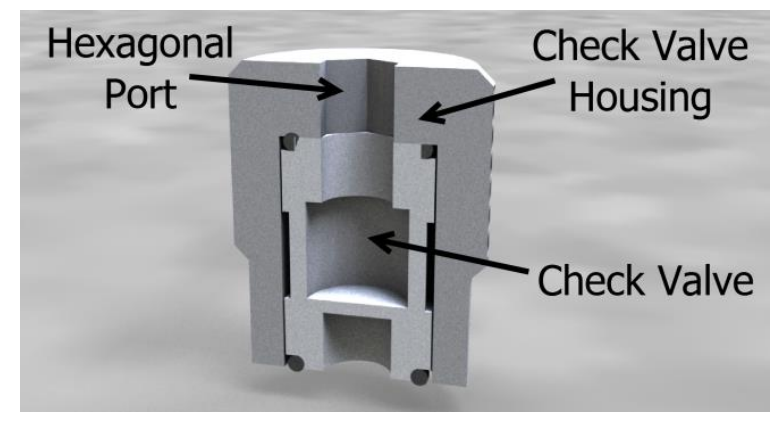

Figure 3.9 - Check valve seated within the proper check valve housing. The housing increased the size of the bore required in the manifold but ensured that the check valve remained seated and sealed. 


\subsubsection{Circuit and Manifold Design}

The orignal soft switch design had locations for air to be trapped both in front and behind the piston, in the internal piston tank port, and in the internal lines to load and tank. In order to bleed all of the air out of the original design, an external pump was connected to the tank port, and fluid was forced throughout the manifold while plugs throughout the manifold were loosened and then replaced. After the bleeding was completed, the manifold was reconnected to the circuit. This approach still allowed air to enter the system during the removal of the manual pump and the insertion of the manifold in the VVDP circuit. This approach was time consuming, did not guarantee that air was removed from the system, and had the possibility of having air reenter the circuit during reassembly.

A redesign of the bleeding sequence and bleed ports, focused on allowing for bleeding of the manifold with it remaining in place within the circuit. It was determined that the pressure transducer ports at the front and back of the piston could also function as bleed ports. In order to ensure that the air was removed from all cavities within the manifold, a method for forcing fluid through the internal tank port needed to be determined. The solution was to install two ball valves into the circuit, one just before the tank and the other after the tank check valve as seen in Fig. 3.10. Ball valves were an ideal choice since when they are in the open position their design adds negligible throttling losses to the system. Closing the ball valve before the tank and switching the 3way valve to tank, forced fluid through the tank check valve and into the cavity behind the soft switch piston before forcing it through the pressure check valve and load line to tank. At this point, air was bled from both the front and back of the soft switch piston by loosening the pressure transducer connections. Rapidly closing and opening the other ball valve forced fluid through the internal piston port to tank, allowing the remainder of the air to be bled from the system. 


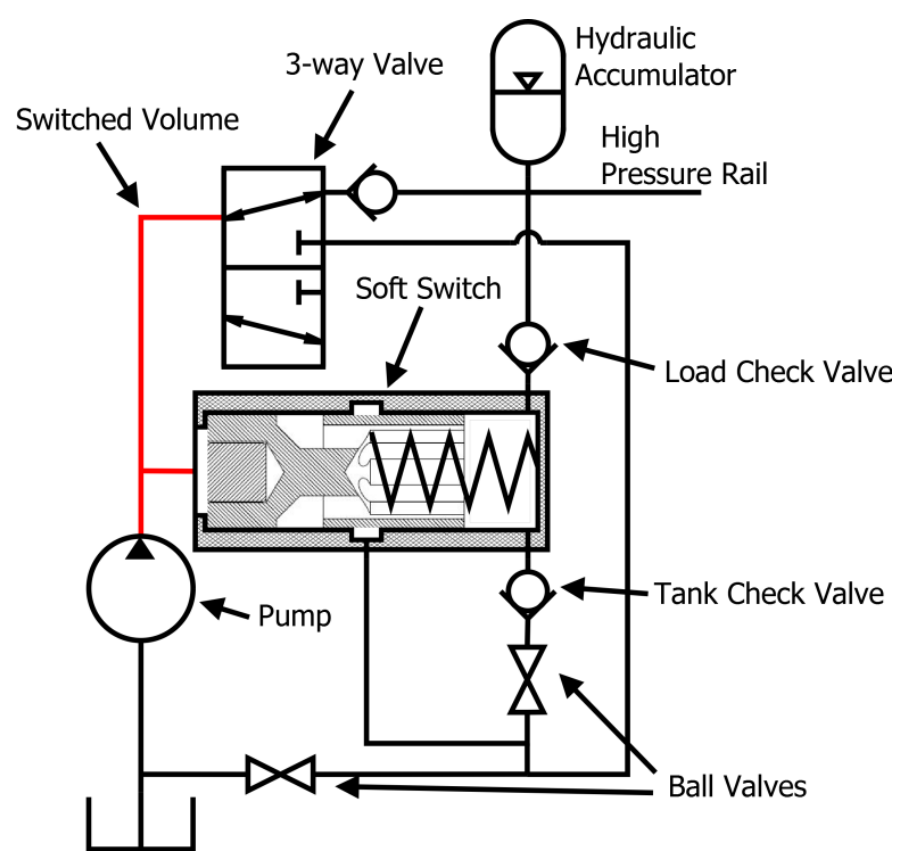

Figure 3.10 - The bleeding sequence for the soft switch manifold was carried out by strategically opening and closing the ball valves to force fluid through the cavities throughout the manifold.

To make the bleeding sequence faster and less laborious, the bleed ports used by the pressure transducers were tooled as O-ring boss (ORB) ports. Off the shelf fittings for converting the national pipe thread (NPT) pressure transducers to an appropriate ORB size added significant switched and dead volume to the circuit. Simple custom fittings were manufactured that reduced the switched and dead volumes beyond what even most ORB threaded pressure transducers could achieve.

The manifold was made out of aluminum so as not to interfere with the magnetic piston displacement sensor. In order to reduce the size of the switched volume in the circuit, the bleed/pressure transducer port at the front of the soft switch was made as small as possible. The two check valve ports were manufactured so that the check valves could seal against the bottom of the bores, and ORB fittings could be used to connect the manifold to the rest of the circuit. Notice in Fig. 3.11 that all of the ports to and from the manifold are separate from each other, which made troubleshooting easier but also forced more attention to be paid to the space required to thread all of the proper fittings into 
place. Though some of the clearances between fittings were tight, there was adequate space for all of the connections to be made.

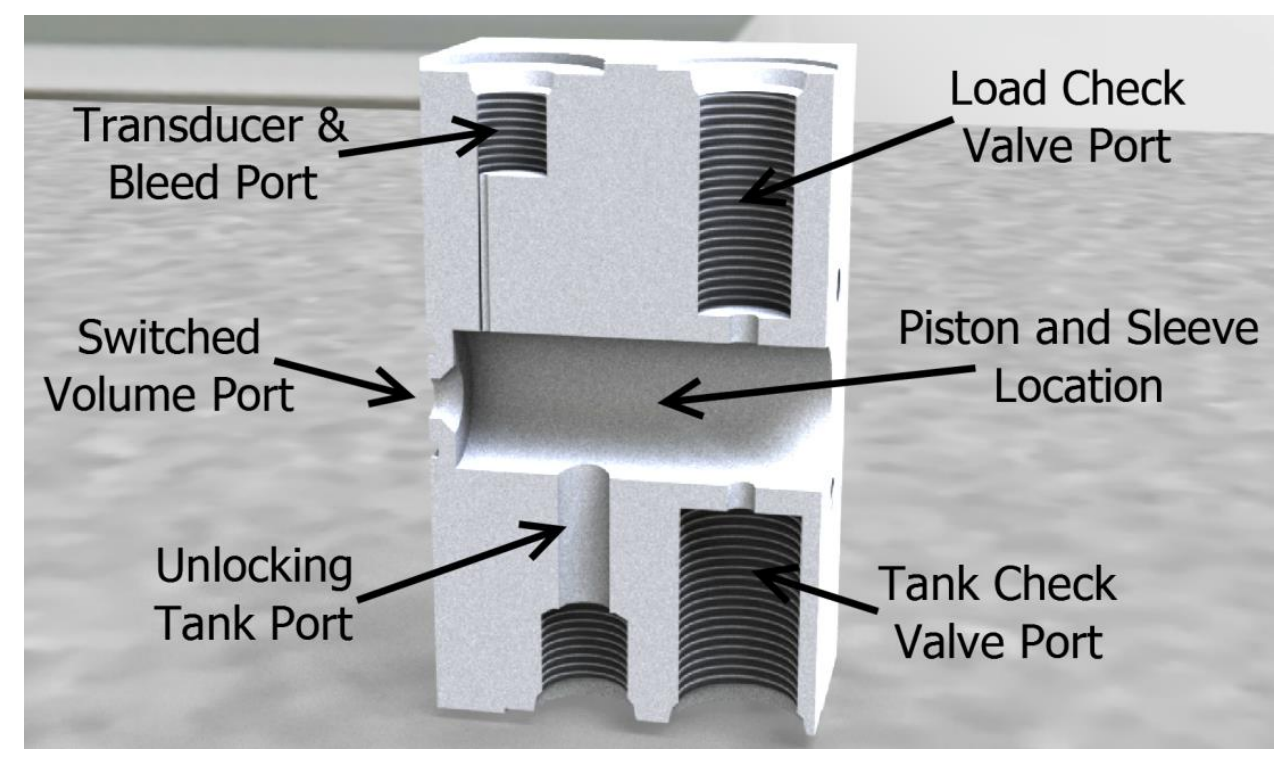

Figure 3.11 - Cross-sectional view of the soft switch manifold, showing the proximity of the ports to each other. Though the clearances were tight, there was adequate space for the requisite fittings, while still allowing check valves to be centered and seated properly.

The size of the tank check valve bore and threads left a small wall thickness on the back of the manifold where it mates with the face plate, but since the port only experienced low tank pressure, the wall thickness was of no concern. A small groove was also designed next to the piston bore which allowed for close proximity of the position sensor (Fig. 3.12). The detail design of the soft switch manifold can be seen in Fig. A.1 of Appendix A. 


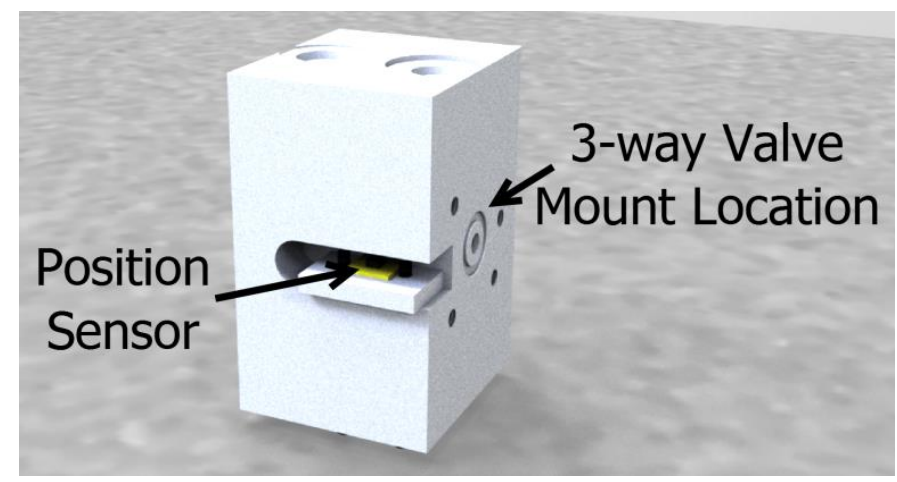

Figure 3.12 - Soft switch manifold viewed from the reverse side. The groove for the position sensor mounting plate allowed the position sensor to displace next to the axis of the piston, in order to properly center the sensor.

\subsection{Experimental Setup}

\subsubsection{Components and Layout}

The test stand for the soft switch circuit is shown in Fig. 3.13. It consisted of a $1.49 \mathrm{~kW}$ AC motor $(A)$ driving a variable displacement axial piston pump $(B)$ which had the pressure compensator on it defeated so that it behaved as a fixed displacement source. The PWM of the circuit was provided by a 3-way valve and accompanying solenoid $(C)$ which was switched to load when not energized. The soft switch manifold $(D)$ was mounted directly to the 3-way valve manifold which was connected directly to the pump to reduce the system switched volume. The 3-way valve load port connected to a check valve $(E)$, a 1.0L diaphragm accumulator $(F)$ pre-charged to $4.27 \mathrm{MPa}$, a gear flow meter $(R)$, a proportional pressure relief valve $(G)$ powered by a 30V DC power supply $(H)$

which provided the system load, and a cooling coil $(S)$, before returning to tank. Load and tank check valves $(I)$ were located at the back of the soft switch to provide the unlocking signal and piston reseating flow respectively. Located in the tank lines were the two ball valves $(J \& K)$ for bleeding air out of the system. A Coriolis flow meter $(L)$ determined the flow rate into the pump, while a position sensor $(M)$ determined the axial position of 
the soft switch piston. Three 20.7 MPa pressure transducers gathered switched volume pressure $(N)$, pressure behind the soft switch piston $(O)$, and load pressure $(P)$, while a 20.7 MPa pressure gauge $(Q)$ allowed cyclic stead-state load pressure to be determined. Component part numbers and specifications can be cross-referenced in Table 3.2, with clarification regarding the layout of the hydraulic portion of the test circuit is provided by Fig. 3.14.

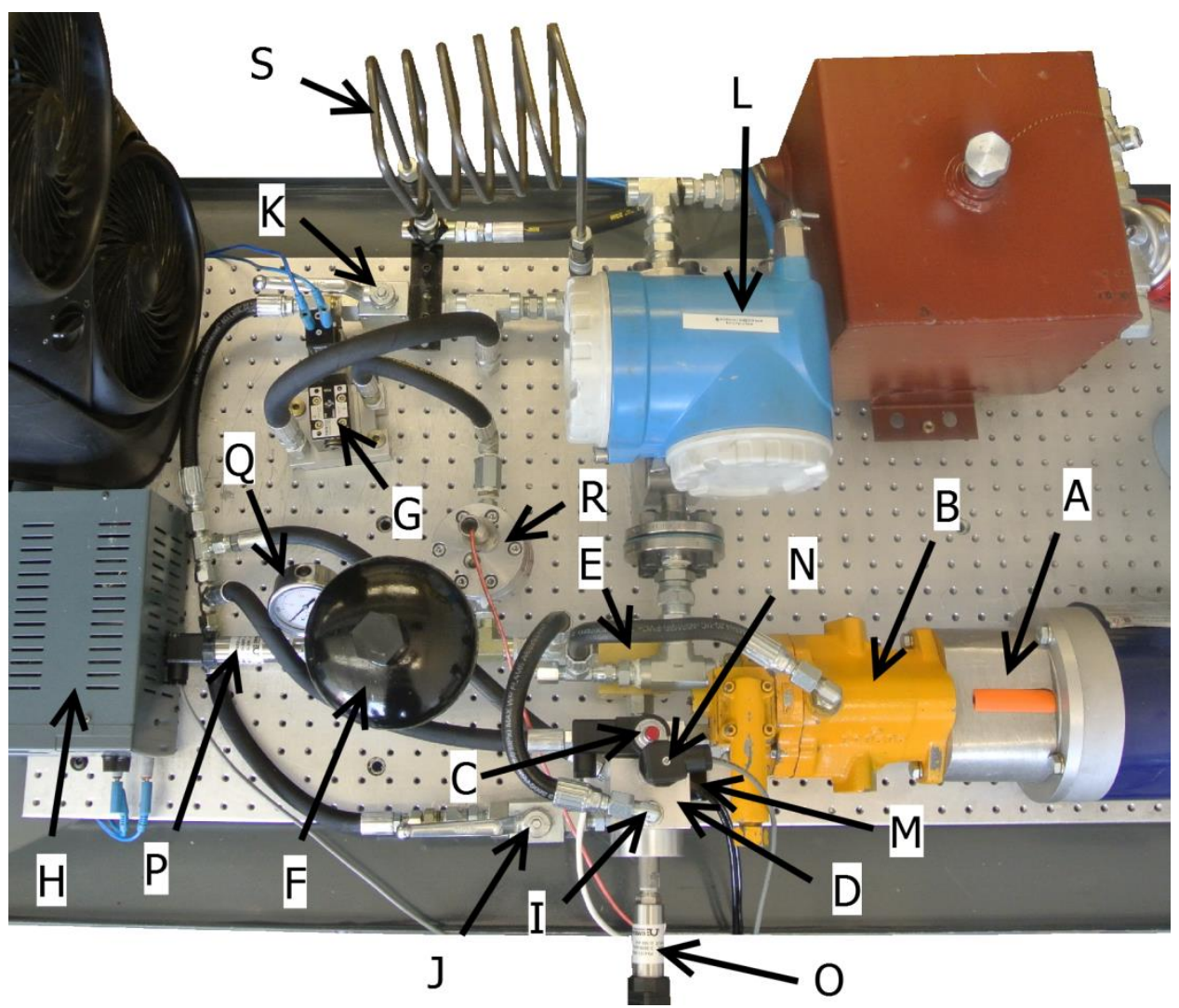

Figure 3.13 - Soft switch test bed setup with component labels. 
Table 3.2 - Component part numbers and specifications for soft switch test bed components.

\begin{tabular}{clccc}
\hline \hline Label & Name & Make & Model & Specs. \\
\hline A & Motor & Industrial Duty & T2-18-56CB & $2 \mathrm{HP}, 1725 \mathrm{RPM}$ \\
$\mathrm{B}$ & Var. Disp. Pump & & & $11+\mathrm{L} / \mathrm{min}$ \\
$\mathrm{C}$ & 3-way Valve & Hydroforce & SV08-30 & $20.7 \mathrm{MPa}$ \\
$\mathrm{D}$ & Soft Switch & & & \\
$\mathrm{E}$ & Load Line C. V. & Eaton & CV6-10 & $76 \mathrm{~L} / \mathrm{min}, 35 \mathrm{MPa}$ \\
$\mathrm{F}$ & Accumulator & Parker & AD100B20T9A1 & $1 \mathrm{~L}, 20 \mathrm{MPa}$ \\
$\mathrm{G}$ & Prop. Pres. Relief & Festo Didactic & 151456 & $8 \mathrm{~L} / \mathrm{min}, 12 \mathrm{MPa}$ \\
$\mathrm{H}$ & DC Power Supply & Extech & 382213 & $30 \mathrm{Volts} / 3 \mathrm{Amps}$ \\
$\mathrm{I}_{\mathrm{a}}$ & Load C.V. & Inserta & ICS-C-02-N015 & $7.57 \mathrm{~L} / \mathrm{min}, 103 \mathrm{kPa}$ \\
$\mathrm{I}_{\mathrm{b}}$ & Tank C.V. & Inserta & ICS-C-04-N003 & $18.9 \mathrm{~L} / \mathrm{min}, 20.7 \mathrm{kPa}$ \\
$\mathrm{J} / \mathrm{K}$ & Ball Valve & & & \\
L & Coriolis Flow Meter & Endress+Hauser & Promass 80E08 & $10 \mathrm{MPa}$ \\
$\mathrm{M}$ & Position Sensor & Honeywell & HMC1512 & $120 \mathrm{mV}$ \\
$\mathrm{N} / \mathrm{O} / \mathrm{P}$ & Pressure Transducer & Omega & PX4201 & $20.7 \mathrm{MPa}, 100 \mathrm{mV}$ \\
$\mathrm{Q}$ & Pressure Gauge & & & $20.7 \mathrm{MPa}$ \\
$\mathrm{R}$ & Gear Flow Meter & AW Gear & JVA-20KG-25 & $7.57 \mathrm{~L} / \mathrm{min}, 34.5 \mathrm{MPa}$ \\
$\mathrm{S}$ & Cooling Coil & & & \\
\hline \hline
\end{tabular}




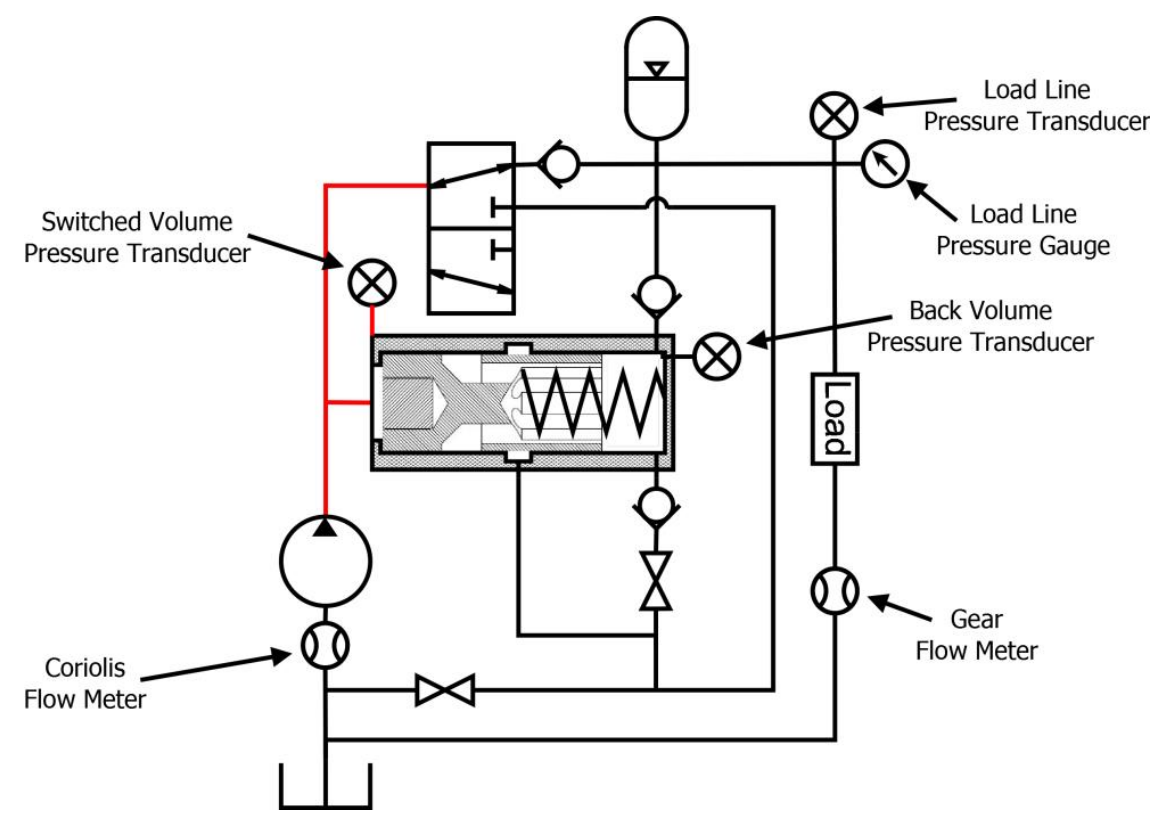

Figure 3.14 - The soft switch hydraulic test circuit layout. Three pressure transducers and two flow meters gathered pertinent information regarding system behavior.

Originally the Coriolis flow meter was placed in the tank line after the 3-way valve so that the dynamics of both the load and tank line flows could be seen, and so that the flow rate through the pump could be found by the addition of the two flows. It was later determined that since the two flows required when calculating system efficiency are the pump flow rate and the load flow rate, those two quantities should be found directly to ensure the accuracy of the calculations. As long as the pump operated at low displacement and the fluid reservoir was full, the location of the Coriolis flow meter did not result in cavitation. To further ensure that the measured pump flow rate was accurate, the case drain for the pump was rerouted to a location between the flow meter and the pump, instead of back to the reservoir.

During initial soft switch testing the load for the circuit was provided by a needle valve. The pressure at the load was a function of pump flow rate as well as duty ratio and switching frequency of the 3-way valve. During testing, those parameters were adjusted repeatedly to determine the operating range of the soft switch. This resulted in repeated needle valve adjustments to reach proper load parameters. As a result, it was decided that 
the needle valve should be replaced with a more active load control, so a proportional pressure relief valve was used in that location instead.

The fluid used in the test circuit was Mobil DTE 25 (ISO 46) which at $40{ }^{\circ} \mathrm{C}$ has a

mass density of $876 \mathrm{~kg} / \mathrm{m}^{3}$, and a dynamic viscosity of $0.0387 \mathrm{~Pa}$ s. As the temperature of the oil heats up, the properties of the oil change. Since the temperature of the oil increases during PWM, a fan was placed in a location that allowed it to be turned on to help maintain the system temperature during testing. Testing occurred at a temperature of approximately $52.5^{\circ} \mathrm{C}$ which corresponds to a dynamic viscosity of approximately $0.0275 \mathrm{~Pa}$ s for an ISO 46 fluid [39].

\subsubsection{System Control and Data Acquisition}

The control and data acquisition for the test bench was completed using two instances of Matlab. One instance controlled the PWM of the 3-way valve, while the other gathered all of the sensor data for each test. The electrical circuit for the valve control can be seen in Fig. 3.15. The $720 \Omega$ resistance ensured that damage would not occur via a large current draw, while the $4.7 \mathrm{k} \Omega$ resistance between the logic and ground acted as a pull-down resistor. When controlling an inductance device, a freewheeling diode is placed in parallel with the inductor. This diode provides a path for the current when the coil is switched off, avoiding a voltage spike that could harm the transistor. 


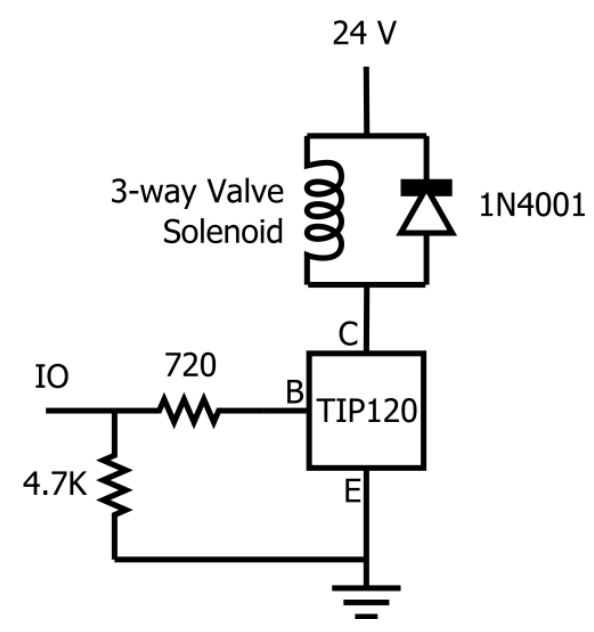

Figure 3.15 - Solenoid driver circuit for 3-way valve.

The sensor used to measure the position of the soft switch piston has magnetoresistive components placed in a wheatstone bridge configuration, which is used to determine the direction of an applied magnetic field. Since the sensor only senses the direction of the magnetic field, and not the amplitude, it can be placed next to a short axially polarized magnet and easily determine the magnetic field direction changes as the magnet translates axially (Fig. 3.16). If placed near the end of the magnet, the changes in the magnetic field direction will be large enough to provide adequate resolution when correlated to position. The two wheatstone bridges in the sensor are rotated 45 degrees with respect to each other, providing a larger axial sensing range by allowing unique output for a larger displacement. The sensor was calibrated by placing the soft switch piston in known locations within its displacement range and correlating that position with the combined magnetic field orientation data from the sensor bridges. A curve fit was then done that would output the piston position throughout the piston range. 
HMC1512

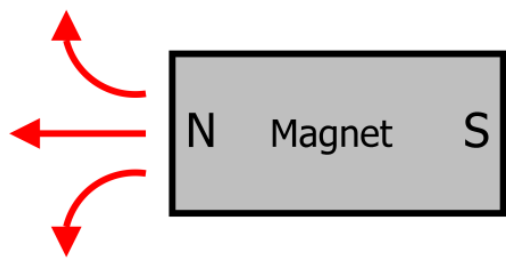

Figure 3.16 - Magnetoresistive position sensor orientation in relation to a magnet.

During initial testing of the soft switch circuit, it appeared that the soft switch piston was displacing early, as seen by Triana in the initial prototype [33]. It was eventually determined that the piston was displacing as expected, but due to the compact design of the soft switch manifold, the solenoid for the 3-way valve was interfering with the magnetic field as detected by the position sensor. Because the disturbance was cyclic and predictable it was easily removed from the position data. To do so, first the 3-way valve was actuated at the desired duty ratio and period with the pump off, while collecting the apparent position data from the sensor. This data was then subtracted from the position sensor data collected with the pump on. Fig. 3.17 shows the piston position data before and after disturbance removal. For the first part of the switching cycle the 3way valve was switched to tank, which means that the solenoid was powered, affecting the magnetic field. This resulted in the large error seen at the beginning of the cycle, which slowly decreased as the coil de-energized. The error slowly returned as the solenoid was powered and the 3-way valve switched back to tank.

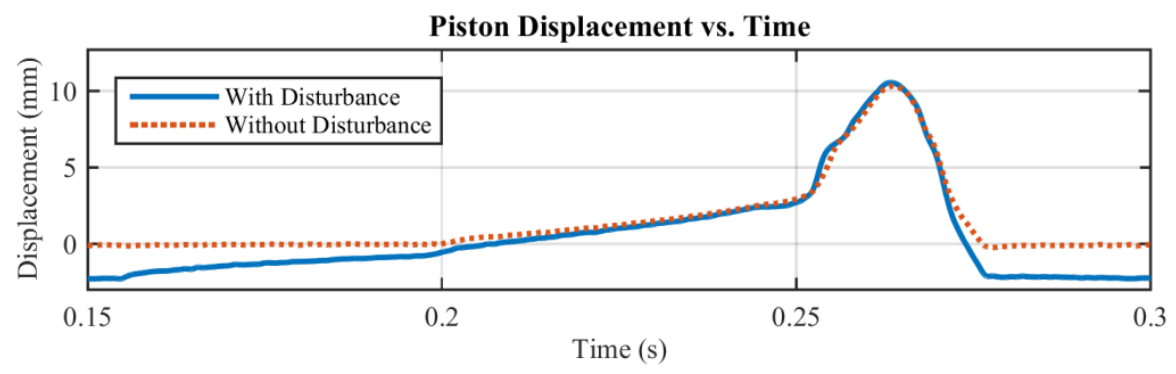

Figure 3.17 - Piston position vs time with solenoid disturbance and after solenoid disturbance removal. 


\subsection{Physical Model Conclusion}

The redesign of the soft switch components as well as the test circuit has been discussed in this chapter. The material incompatibilities were addressed between the soft switch piston and sleeve, and the port sizes and lengths were adjusted to allow for a larger piston displacement. The compression spring preload and piston displacement limiter were redesigned allowing for easier independent adjustment as well as a more controlled range. The check valves will seat and have appropriate cracking pressures to allow for proper pressure signals and piston reseating without cavitation. The test circuit layout was adjusted to allow the system to be bled easily and to ensure more accurate flow measurements. The control and data acquisition systems were also outlined. Chapter 4 shows the operation of the soft switch prototype in detail, comparing it with the predictions of the numerical model from Chapter 2. 


\section{Chapter 4:}

\section{Simulation and Experiment Results}

In this chapter the functionality of the soft switch circuit will be analyzed. Values for the adjustable circuit parameters that allow for proper soft switch operation will be outlined. Potential updates to the numerical model of the circuit will be discussed. Comparisons will be made between the numerical and physical models to demonstrate the numerical model as a design and optimization tool. Finally, the efficiency of the soft switch circuit will be discussed in comparison to other control architectures.

\subsection{Experiments}

To determine whether the soft switch locking mechanism is functioning according to design, some definitions of proper operation are required. As the purpose of the soft switch is to absorb pump flow during the 3-way valve transition to tank, it was determined that proper operation of the soft switch has the following characteristics:

1) The piston does not unlock too early or too late to absorb the pressure spike

2) The piston does not reach the maximum displacement limit

3) The piston reseats prior to the start of the next switching cycle 
The soft switch parameters that were designed to be adjusted during testing are valve duty ratio, switching period, load pressure, pump flow rate, soft switch unlocking distance, maximum soft switch displacement, and soft switch spring preload. To define a baseline case, the physical soft switch parameters were placed near the middle of their adjustment ranges, and the switching period and load pressure were adjusted until the soft switch behaved appropriately, as seen in Table 4.1. It was discovered that at these parameters the piston was always able to reseat prior to a new switching cycle beginning, so the piston displacement limiter was left at the maximum value of $12.7 \mathrm{~mm}$ for all tests. For comparison, a control case was defined, also presented in Table 4.1, where the soft switch was held in the locked position and the valve duty ratio was adjusted to create the same pump flow rate as the baseline soft switch case.

Table 4.1 - Operating parameters of significant interest for the physical model during normal operation.

\begin{tabular}{lcccc}
\hline \hline & & & \multicolumn{2}{c}{ Value } \\
\cline { 3 - 5 } Parameter & Symbol & Units & $\begin{array}{c}\text { Control } \\
\text { Case }\end{array}$ & $\begin{array}{c}\text { Baseline } \\
\text { Case }\end{array}$ \\
\hline Switching period & $T$ & $\mathrm{~s}$ & 0.15 & 0.15 \\
Duty ratio & duty & $\%$ & 52 & 60 \\
Flow duty ratio & duty $_{f}$ & $\%$ & 30.3 & 30.3 \\
Load pressure & $P_{\text {load }}$ & $\mathrm{MPa}$ & 5.63 & 5.62 \\
Pump flow rate & $Q_{\text {pump }}$ & $\mathrm{L} / \mathrm{min}$ & 3.78 & 3.79 \\
Unlock distance & $x_{\text {unlock }}$ & $\mathrm{mm}$ & $\mathrm{NA}$ & 5.08 \\
Spring preload & $F_{S}$ & $\mathrm{~N}$ & $\mathrm{NA}$ & 27.3 \\
\hline \hline
\end{tabular}

Piston displacement, switched volume pressure, and back volume pressure profiles were gathered for a control case where the soft switch piston was locked at a displacement less than the cracking distance, to allow comparison of the VVDP circuit with and without a soft switch (Fig. 4.1). Four time periods are shown in the figure, relating to the position of the control valve during the switching cycle. Time period $t_{1}$ represents the control valve tank port closure, $t_{2}$ represents the opening of the control valve load port, $t_{3}$ indicates the control valve load port closure, and $t_{4}$ represents the opening of the control valve tank port. Notice that the pressure behind the soft switch does not build with the switched volume pressure. The pressure in the switched volume 
builds as expected when the control valve switches to load, and the pressure spikes to 9.02 MPa during the transition back to tank.

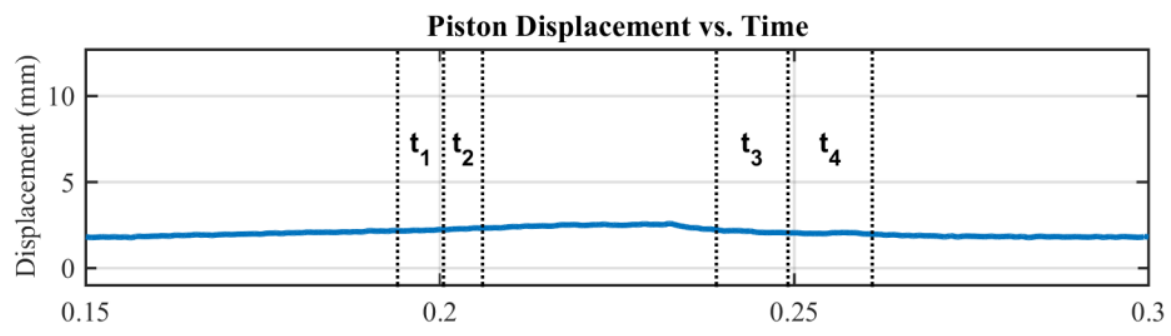

System Pressures vs. Time

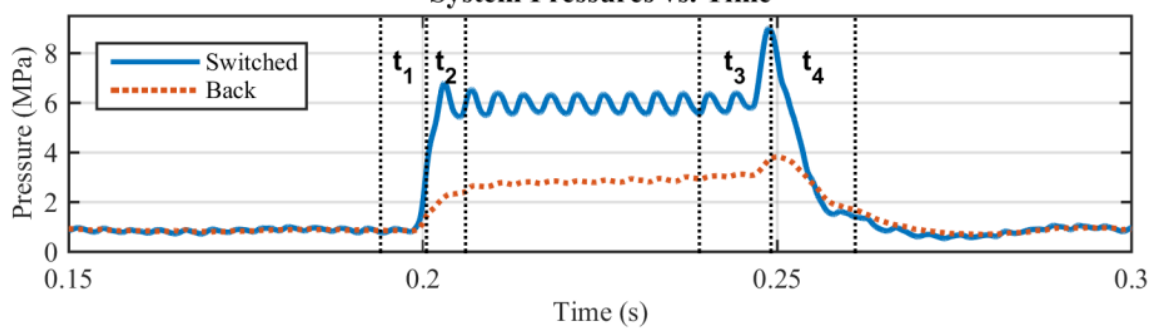

Figure 4.1 - Soft switch piston displacement and pressure profiles for control case. The switched volume pressure spike reaches $9.02 \mathrm{MPa}$.

The piston displacement and pressure profiles of the baseline soft switch case are shown in Fig. 4.2. Notice that when the switched volume pressure begins to build, as a result of the control valve transition to load, the soft switch piston experiences an initial displacement, followed by a fairly steady displacement due to leakage, until the control valve switches back to tank. During the control valve transition to tank, the piston displaces to the unlock position, and the pressure spike in the switched volume is reduced. At the end of the control valve transition, the switched volume returns to tank pressure, allowing the soft switch piston to reseat in preparation for the next cycle. 

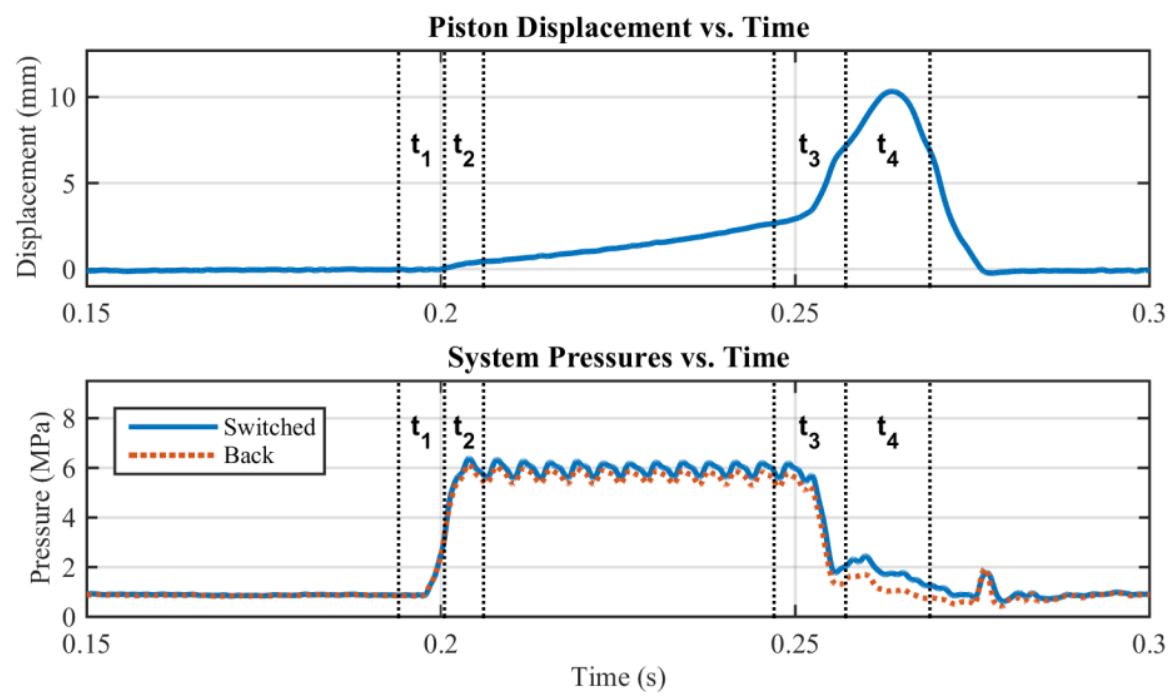

Figure 4.2 - Soft switch piston displacement and pressure profiles for baseline parameters.

The efficiency of the VVDP circuit is one of the ways that test cases using different parameter values can be compared. The efficiency can be calculated by evaluating the ratio of the energy going to load per cycle to the energy delivered by the pump per cycle:

$$
\eta=\frac{\int_{0}^{T} P_{\text {load }} Q_{H S V, l} d t}{\int_{0}^{T} P_{\text {switch }} Q_{\text {pump }} d t}
$$

where $P_{\text {load }}$ is the constant load pressure, $Q_{H S V, l}$ is the flow rate through the control valve to load, $P_{\text {switch }}$ is the pressure in the switched volume, and $Q_{\text {pump }}$ is the constant pump flow rate. If the soft switch is unlocking correctly, a small portion of the flow that normally goes to the load in a PWM system without a soft switch will be absorbed by the soft switch and then flow to tank. This effectively reduces the duty ratio of the circuit. In order to properly compare the efficiencies of the different test cases using Eq. 4.1, their flow duty ratios, which are defined as the flow rate reaching the load divided by the flow rate of the pump, need to be the same. This necessitates adjustments of the valve duty ratio for each test case. 
While adjusting the valve duty ratio to maintain the flow duty ratio, the soft switch parameters were adjusted one by one to determine their influence on the soft switch performance. A summary of those parameter values can be seen in Table 4.2.

Table 4.2 - The soft switch test parameters. Flow duty ratio is maintained by adjusting the duty ratio of the 3-way valve.

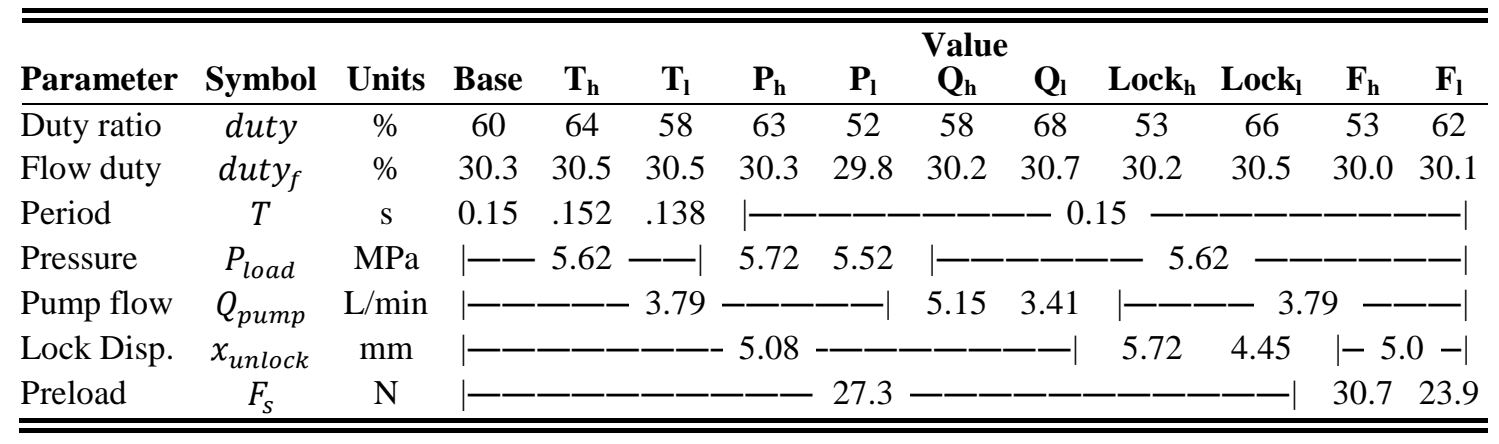

The system temperature for all of the tests was maintained at $52.5{ }^{\circ} \mathrm{C} \pm 2.5{ }^{\circ} \mathrm{C}$ with the accumulator charged to $4.27 \mathrm{MPa}$. The effects of the parameter changes on piston displacement and system pressures can be seen in Figs. 4.3 - 4.7.

\section{Control Valve Switching Period}

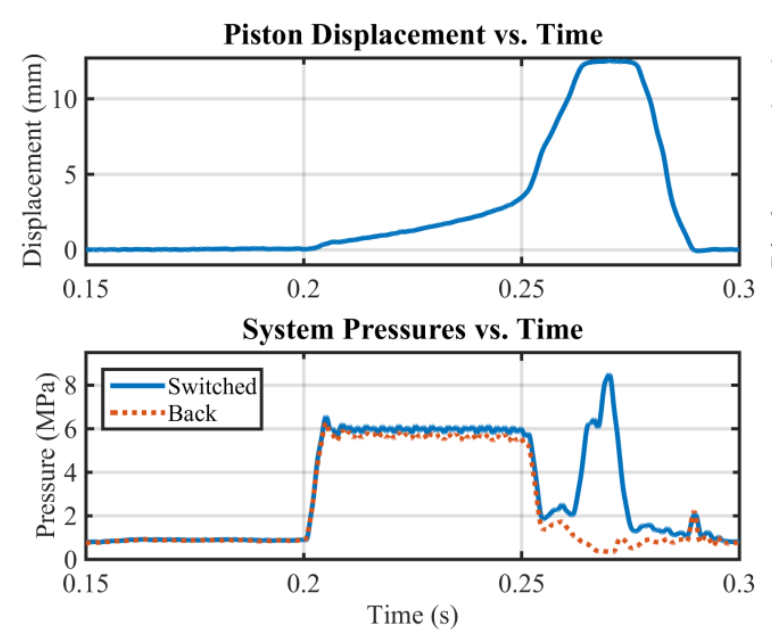

(a) High control valve switching period of .152 seconds.

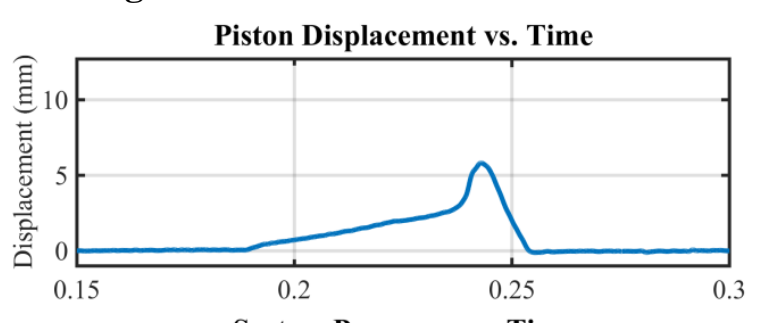

System Pressures vs. Time

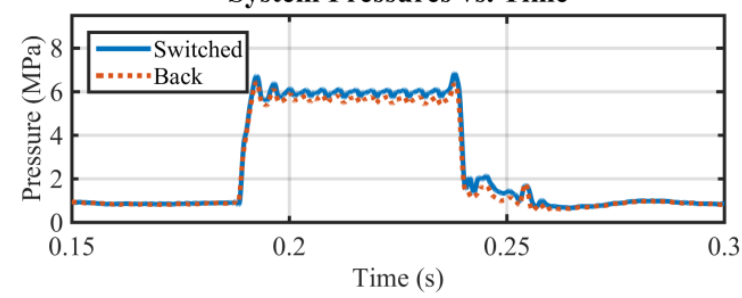

(b) Low control valve switching period of .138 seconds.

Figure 4.3 - Control valve switching period adjustment. 
Load Pressure
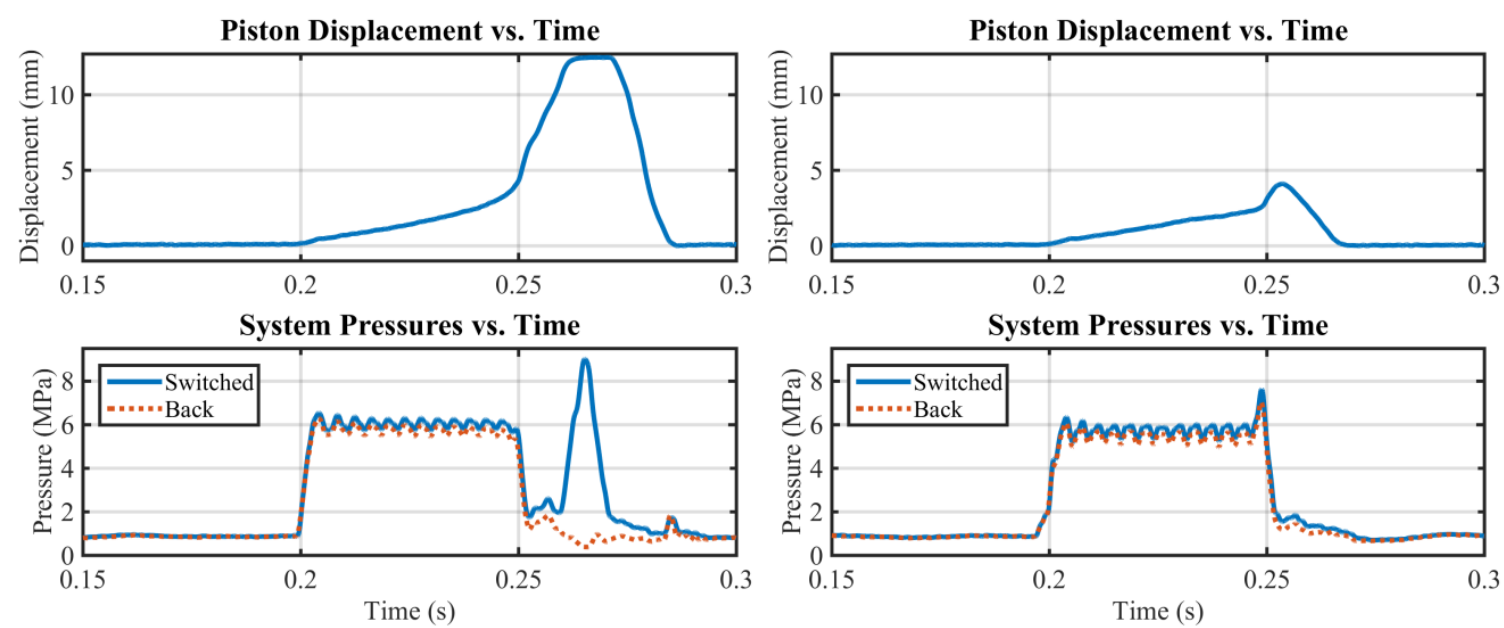

(a) High load pressure of $5.72 \mathrm{MPa}$.

(b) Low load pressure of $5.52 \mathrm{MPa}$.

Figure 4.4 - System load pressure adjustment.

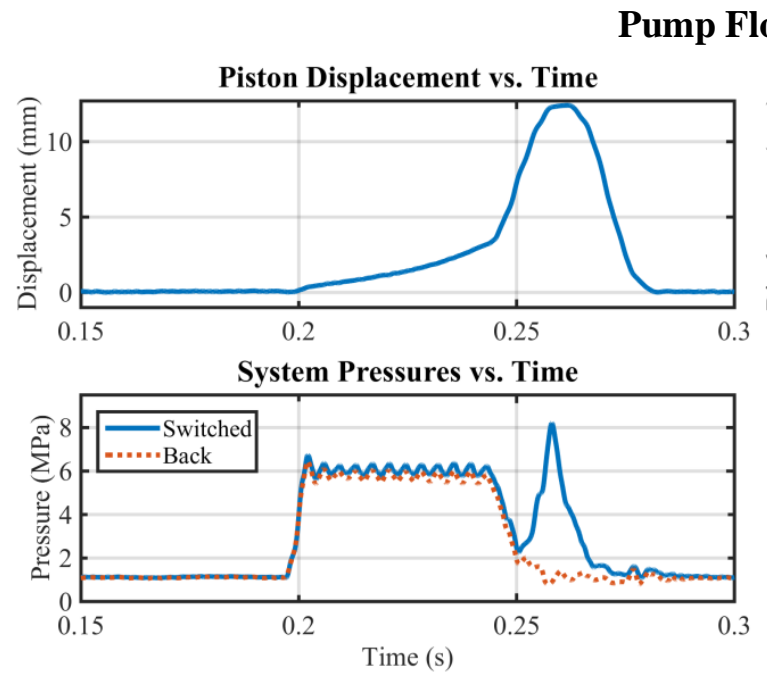

(a) High pump flow rate of $5.15 \mathrm{~L} / \mathrm{min}$.
Piston Displacement vs. Time

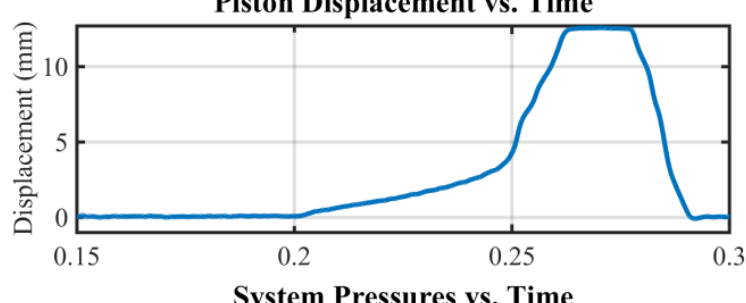

System Pressures vs. Time

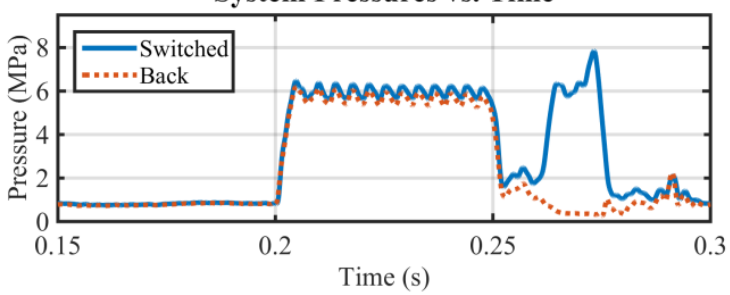

(b) Low pump flow rate of $3.41 \mathrm{~L} / \mathrm{min}$.

Figure 4.5 - Pump flow rate adjustment. 


\section{Soft Switch Unlocking Distance}
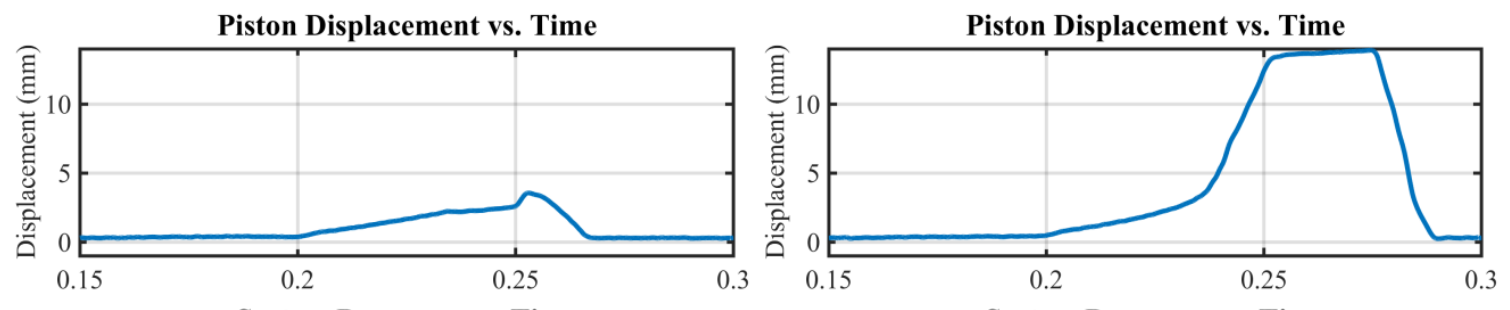

System Pressures vs. Time

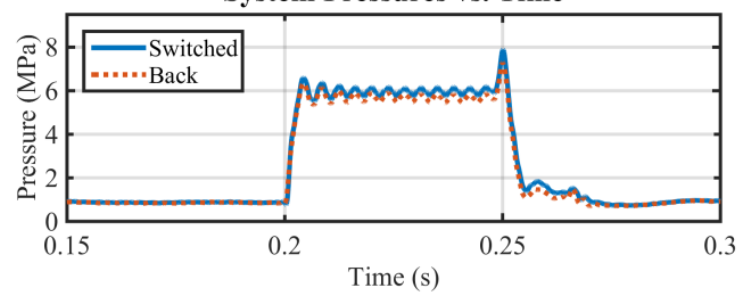

(a) High soft switch unlocking distance of $5.72 \mathrm{~mm}$.

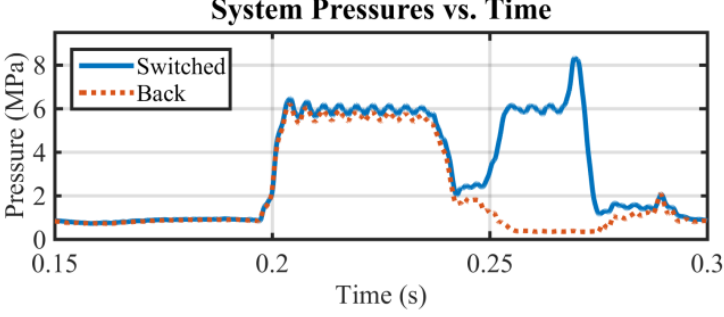

(b) Low soft switch unlocking distance of $4.45 \mathrm{~mm}$.

Figure 4.6 - Soft switch unlocking distance adjustment.

\section{Soft Switch Spring Preload}
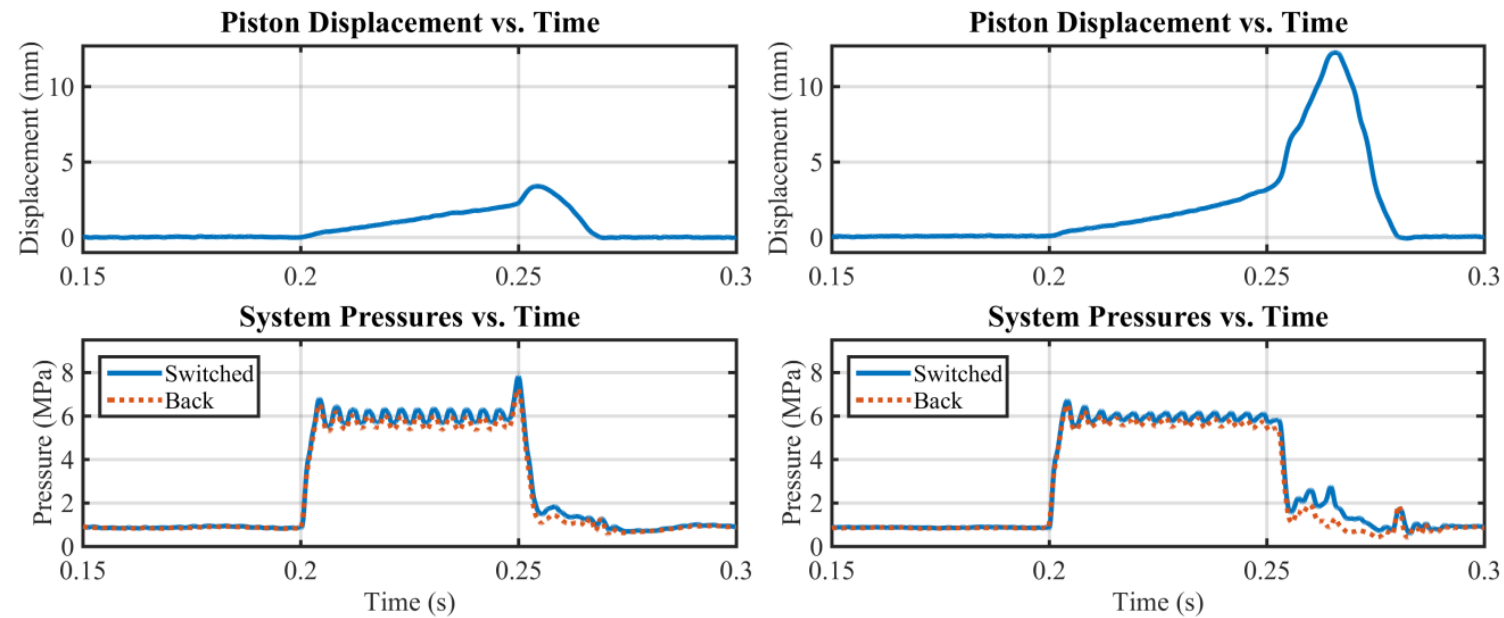

(a) High soft switch spring preload of $30.7 \mathrm{~N}$.

(b) Low soft switch spring preload of $23.9 \mathrm{~N}$.

Figure 4.7 - Soft switch spring preload adjustment. 
For the switching period, load pressure, and pump flow rate adjustments, the high parameter cases lead to the soft switch unlocking early in the switching cycle, while the low cases for the unlocking distance and spring preload parameters leads to an early soft switch unlock. This can be seen in the switched volume pressure spike that occurs after the pressure is initially reduced by the soft switch, as well as in the soft switch piston reaching the maximum displacement limiter at $12.7 \mathrm{~mm}$. Notice that when the soft switch unlocks early enough that the pressure spike is not reduced, it is larger than the pressure spike for the cases where the soft switch fails to unlock (except for the low spring preload case, where the unlock occurred only early enough for the piston to barely reach maximum displacement). The magnitudes of the switched volume pressure spike, due to the early unlock are shown in Table 4.3. These values suggest that the system performance is more sensitive to switching period and load pressure.

Table 4.3 - Switched volume pressure spike for the adjusted parameters. Compare to load pressure of 5.5 to $5.7 \mathrm{MPa}$.

\begin{tabular}{lccccc}
\hline \hline & \multicolumn{4}{c}{ Parameter Pressure Spike (MPa) } \\
\cline { 2 - 6 } Case & $\begin{array}{c}\text { Switching } \\
\text { Period }\end{array}$ & $\begin{array}{c}\text { Load } \\
\text { Pressure }\end{array}$ & $\begin{array}{c}\text { Pump } \\
\text { Flow Rate }\end{array}$ & $\begin{array}{c}\text { Unlock } \\
\text { Distance }\end{array}$ & $\begin{array}{c}\text { Spring } \\
\text { Preload }\end{array}$ \\
\hline Control & & & 8.94 & & \\
Baseline & & & 2.43 & & \\
\hline Early Unlock & 8.51 & 9.03 & 8.22 & 8.35 & 2.77 \\
Late Unlock (except Flow) & 6.86 & 7.64 & 7.86 & 7.89 & 7.83 \\
\hline \hline
\end{tabular}

For the cases where the soft switch unlocked late in the switching cycle, or failed to unlock at all, it can be seen that the switched volume pressure spike still occurs but not at the same magnitude as the early unlock cases. This suggests that though the piston does not reach the displacement necessary to unlock completely, it is still effective at decreasing the magnitude of the switched volume pressure spike through displacement prior to unlock. The magnitude of the switched volume pressure spike, due to late unlock is also shown in Table 4.3. The switched volume pressure spike for the low switching period adjustment is much smaller than the other late unlock pressure spikes, suggesting that the system is more sensitive to switching period adjustments than the other parameters. 


\subsection{Model Updates}

To do a worthwhile comparison between the model and physical system, the model needs to be updated to reflect the minor changes made to the system parameters during the soft switch design. Further model changes were also attempted in order to find greater agreement between the model and prototype.

\subsubsection{Model Robustness and Parameter Updates}

A comparison between the numerical model and baseline case piston displacement shows a large disagreement between the timing of events in the switching cycle, as well as shape and magnitude of those events (Fig. 4.8). Many of the differences can be ascribed to changes in the system parameters between the two cases, which will be discussed and addressed later. Other differences can be remedied through changes to the numerical model itself.
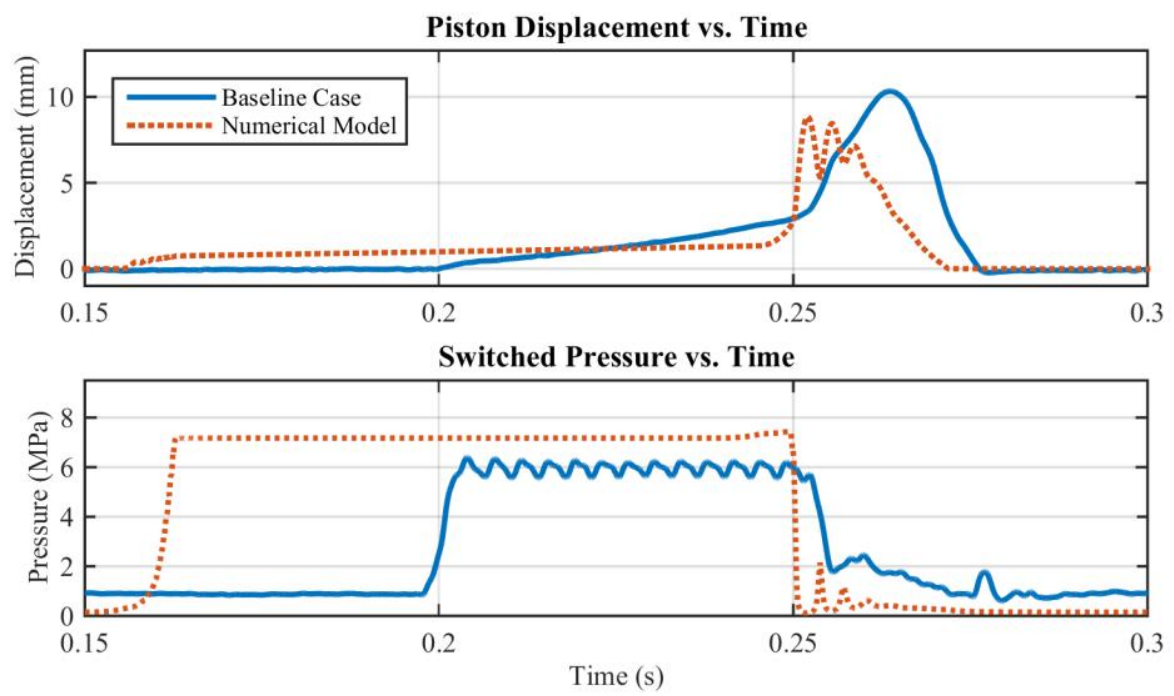

Figure 4.8 - Comparison between the numerical model and the baseline test case, showing the differences in control valve transition event timing, switched pressure, and piston displacement profile. 
One of the large differences between the model and the prototype function is the rate at which the soft switch piston displaces when unlocking. This difference is easily seen in the aforementioned figures, especially in the piston vibration of the model which is not seen in the physical system. Though the energy loss through the internal piston ports is small, as shown in Fig. 3.7, there is enough damping through the ports to significantly affect the piston movement. To address this difference, Eq. 2.19 is adjusted so that the flow through the unlocking port to tank travels through two orifices in series (as in Eq. 2.16). The first orifice is the fixed internal piston port area, and the second is the variable port area due to the piston displacing to the unlock location:

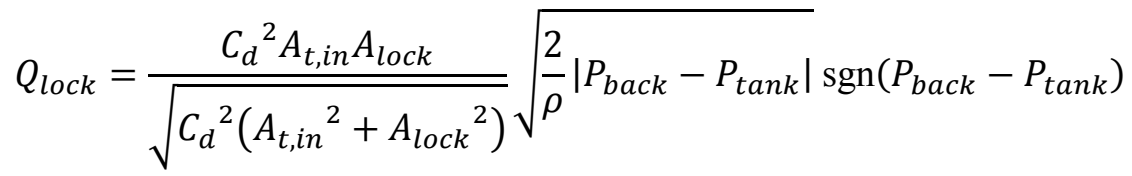

where $A_{t, i n}$ is the area of the piston internal tank port. The discharge coefficients were left at the standard 0.6 value, but as the fluid flow enters the internal tank ports, it is partially blocked by the soft switch spring, effectively decreasing the internal port area by $45.7 \%$. This change in area provides the appropriate amount of damping to the piston.

Another change that was made to the model that significantly improved the agreement with the experimental results was the addition of a delay in the time that a signal was sent to the control valve and the time when the valve spool responded. Eq. 2.2, which details the timing of the control valve, was adjusted as:

$$
\left\{\begin{array}{llll}
\text { if } t \bmod T<l_{d} & s=s_{-1}+\frac{x_{\max }}{t t_{t}} \Delta t & \text { if } s \geq x_{\max } & s=x_{\text {max }} \\
\text { elseif } t \bmod T<t_{\text {off }} & s=s_{-1}-\frac{x_{\max }}{t t_{l}} \Delta t & \text { if } s \leq 0 & s=0 \\
\text { elseif } t \bmod T<t_{\text {off }}+t_{d} & s=s_{-1} & \\
\text { else } & s=s_{-1}+\frac{x_{\max }}{t t_{t}} \Delta t & \text { if } s \geq x_{\max } & s=x_{\max }
\end{array}\right.
$$

where $l_{d}$ and $t_{d}$ are the delays between the load and tank command signals (respectively) being sent to the control valve and the valve spool responding. A load delay of 44 milliseconds allows the initial displacement of the soft switch piston to line up between the model and prototype. Choosing an appropriate tank delay is more difficult, since the 
data from the prototype does not allow for an easily identifiable start to the transition event.

In a paper by Yudell and Van de Ven, the control valve used in the soft switch circuit was characterized in order to create a method of identifying solenoid valve transition events [40]. In an unloaded ambient temperature and pressure test case, the delay between the tank transition signal and the coil being fully energized for transition was 10.23 milliseconds, while the delay for de-energizing was 63.19 milliseconds. Applying the ratio of energizing to de-energizing found by Yudell and Van de Ven, to the load delay of 44 milliseconds found under this loading, leads to a tank delay of just over 7 milliseconds. Using a delay of 7 milliseconds brings the model into better agreement with the prototype baseline case. The last major change is an adjustment of the system tank pressure. Though the tank itself is not pressurized, the cooling coils and other fittings appear to induce a tank pressure of approximately $827 \mathrm{kPa}$ for the baseline case, as seen in Fig. 4.2 before the first control valve transition event. This induced tank pressure is the same for all of the test cases, except for the high and low pump flow rate test cases, which are $1.03 \mathrm{MPa}$ and $793 \mathrm{kPa}$ respectively. The effect of these model updates can be seen in Fig. 4.9, in the overlay of "Updated Model 1" on the original design model (Fig. 2.9) and the test data for the baseline case. Notice the improved timing of the piston displacement and the removal of excessive piston bounce after unlocking in "Updated Model 1". 

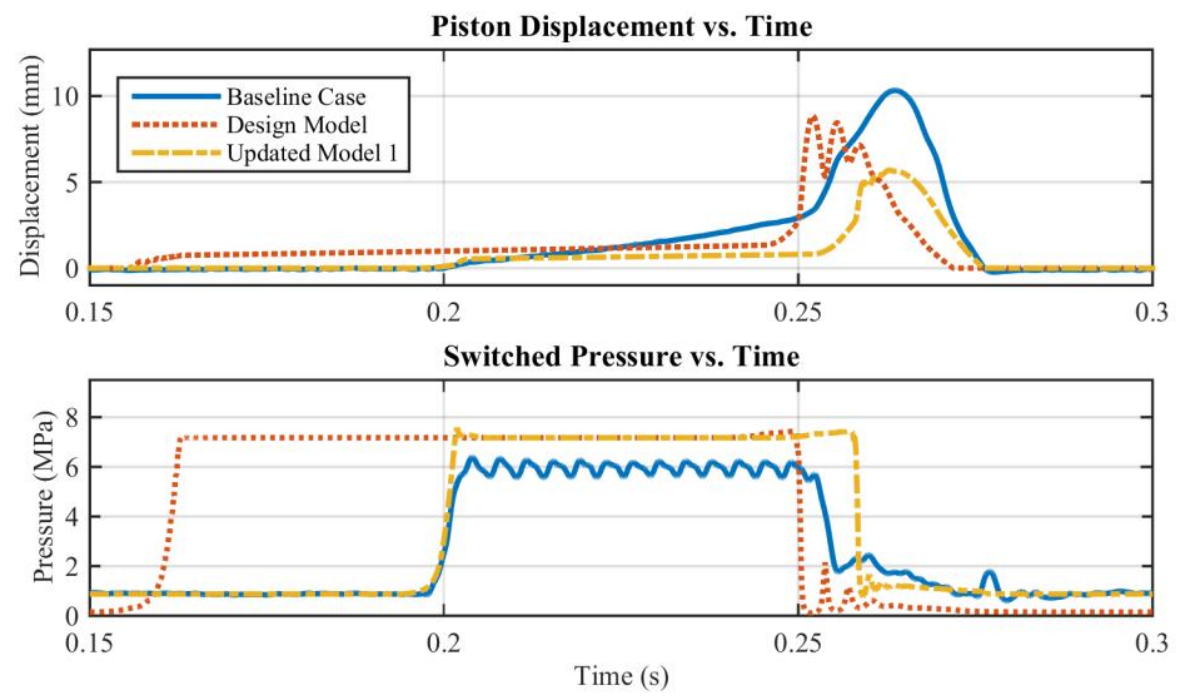

Figure 4.9 - Comparison between the original design model, updated model, and the baseline experimental case showing the effect of 3-way valve spool transition delay and internal piston port to tank flow resistance.

The next differences addressed between the model and experimental data were parameter updates for all of the different system components that were used in the prototype circuit. After repeated testing, a normal operation temperature was determined for the prototype under baseline conditions, which necessitated a change in the dynamic viscosity of the hydraulic oil used in the model from 0.0387 to $0.0275 \mathrm{~Pa}$ s. Since the load pressure was reduced from $6.89 \mathrm{MPa}$ to $5.62 \mathrm{MPa}$ in the prototype testing to reach proper soft switch operation, the model needed to be updated accordingly. The load line check valve had a measured cracking pressure of $124 \mathrm{kPa}$, which was larger than the value of $103 \mathrm{kPa}$ in the model. The discharge coefficient multiplied by the orifice area of the load line check was experimentally determined to be $3.3 \mathrm{~mm}^{2}$, which is less than half as large as the $7.375 \mathrm{~mm}^{2}$ used in the model. The soft switch spring preload and spring rate also need to be adjusted from $22 \mathrm{~N}$ and $5 \mathrm{~N} / \mathrm{mm}$ to $27 \mathrm{~N} 5.38 \mathrm{~N} / \mathrm{mm}$ to reflect the spring chosen for the prototype. The sleeve was shimmed by $2.54 \mathrm{~mm}$, necessitating the change of the piston unlocking distance from $2.54 \mathrm{~mm}$ to $5.08 \mathrm{~mm}$. The effects of these system component parameter updates can be seen as "Updated Model 2" in Fig. 4.10.

Even with the model parameters being adjusted to better represent the physical system, there was still a significant difference in the soft switch unlocking profile. 
Increasing the volume fraction of entrained air to $4 \%$ allowed the model to better reflect the initial soft switch piston displacement that occurred during the control valve transition to load, while remaining inside of the normal range of $2-7 \%$ seen in the literature [41]. A visual inspection of the piston and sleeve showed significant wear to both, and a new measurements of the gap showed an increase in the model gap from $12.7 \mu \mathrm{m}$ to approximately $31 \mu \mathrm{m}$. A slight chamfer was also given to the sleeve tank port holes in order to remove burrs that were leading to wear. This increased the diameter of the holes by approximately $0.5 \mathrm{~mm}$, which in turn decreased the unlocking travel distance of the piston to $4.83 \mathrm{~mm}$. Making these changes to the model allow it to more closely trace the soft switch piston displacement seen in the baseline case. The effects of the above changes can be seen in Fig. 4.10 as "Updated Model 3", and a summary of all of the system parameter changes for the different iterations of the updated model are shown in Table 4.4.
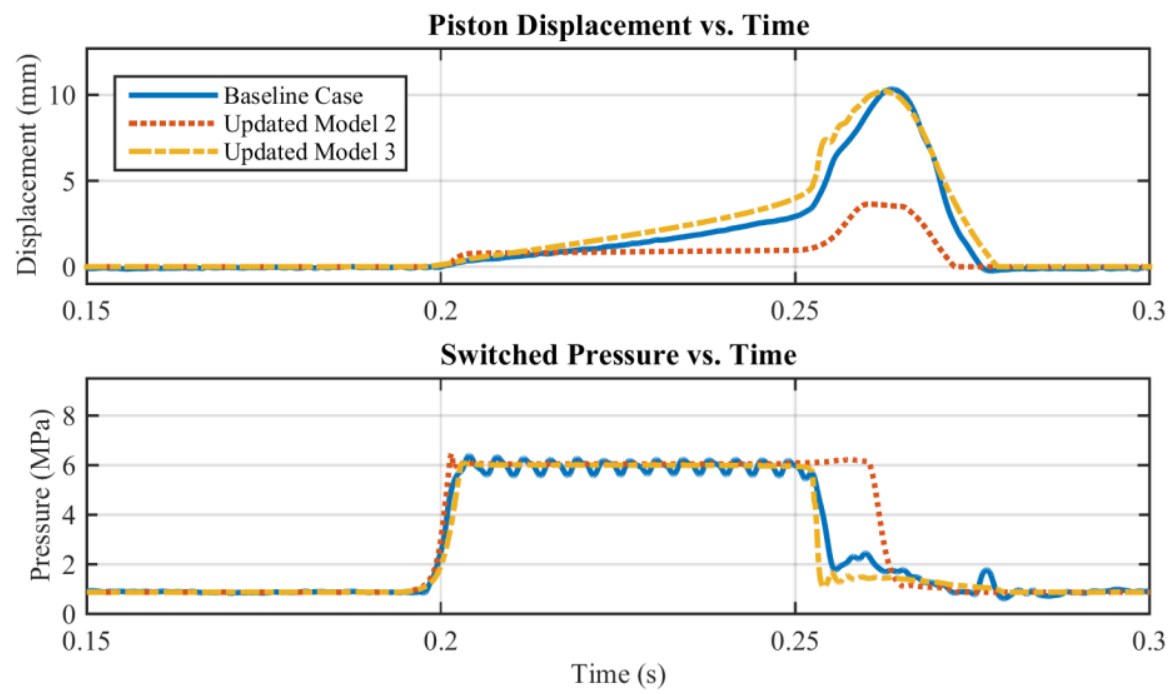

Figure 4.10 - Comparison between the baseline case and the numerical model updates. 
Table 4.4 - System parameter updates to bring the numerical model into agreement with the physical system.

\begin{tabular}{|c|c|c|c|c|c|c|c|}
\hline \multirow[b]{2}{*}{ Parameter } & \multirow[b]{2}{*}{ Symbol } & \multirow[b]{2}{*}{ Units } & \multicolumn{5}{|c|}{ Value } \\
\hline & & & $\begin{array}{l}\text { Design } \\
\text { Model } \\
\end{array}$ & $\begin{array}{l}\text { Updated } \\
\text { Model } 1 \\
\end{array}$ & $\begin{array}{l}\text { Updated } \\
\text { Model } 2 \\
\end{array}$ & & $\begin{array}{l}\text { Jpdated } \\
\text { Model } 3 \\
\end{array}$ \\
\hline Control valve load delay & $l_{d}$ & $\mathrm{~ms}$ & 0 & 1-——- & -44 & & \\
\hline Control valve tank delay & $t_{d}$ & $\mathrm{~ms}$ & 0 & - - & -7 & & \\
\hline Tank pressure & $P_{\text {tank }}$ & $\mathrm{kPa}$ & 101 & |-—— & - 827 & & \\
\hline Dynamic viscosity & $\mu$ & $\mathrm{Pa} \mathrm{s}$ & .0404 & .0387 & & .0275 & 5 \\
\hline Load pressure & $P_{\text {load }}$ & $\mathrm{MPa}$ & |-—- & $6.89---1$ & & 5.62 & - \\
\hline Crack. press. for 1.1. C.V. & $P_{l l}$ & $\mathrm{kPa}$ & & $103---1$ & & 124 & -— \\
\hline Dis. Coeff*Area for C.V. & $C_{d} A_{c h, l l}$ & $\mathrm{~mm}^{2}$ & & 7.37 & & 3.3 & 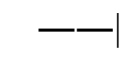 \\
\hline Spring preload force & $F_{S}$ & $\mathrm{~N}$ & & $22.2--1$ & & 27.3 & - \\
\hline Spring rate & $k_{s}$ & $\mathrm{kN} / \mathrm{m}$ & 1 & $5.00--1$ & & 5.38 & 一一 \\
\hline Initial sleeve displacement & $x_{s}$ & $\mathrm{~mm}$ & & --1 & & 2.54 & -1 \\
\hline Dia. sleeve tank port & $d_{s, i n}$ & $\mathrm{~mm}$ & & -5.08 & & & 5.59 \\
\hline Entrained air in switch & $R_{\text {switch }}$ & $\%$ & & -2 & & & 4 \\
\hline Entrained air behind & $R_{\text {back }}$ & $\%$ & & -2 & & & 4 \\
\hline Piston/Sleeve Gap & $c_{p}$ & $\mu \mathrm{m}$ & & --12.7 & & & 31.2 \\
\hline
\end{tabular}

In addition to accurately predicting soft switch behavior for the baseline case, the model is able to accurately predict soft switch piston displacement when the piston unlock occurs early in the switching cycle (Figs. 4.11 and 4.12). Notice that though the soft switch piston initial displacement and unlock timing are accurate for each of these comparisons, the displacement of the piston after it unlocks is slower than prototype tests. This leads to an inaccurate prediction of the switched volume pressure spike magnitude and timing, which affects efficiency predictions. 

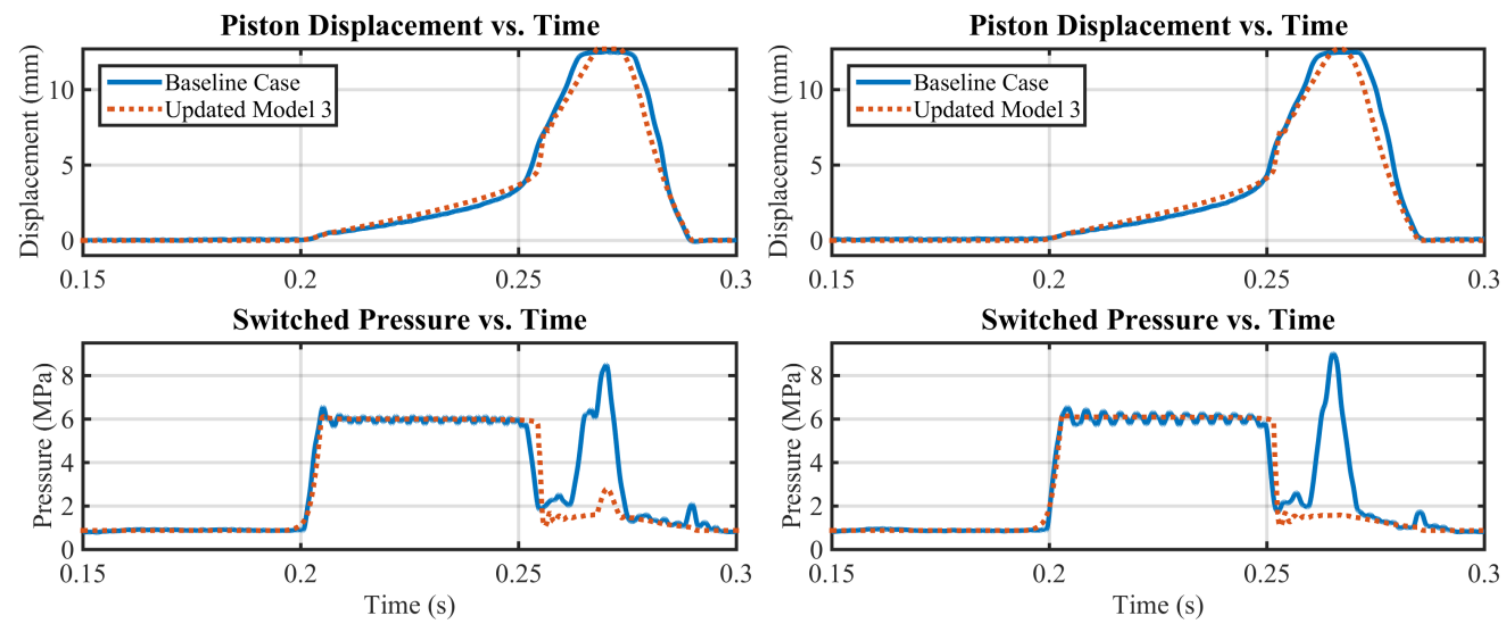

(a) High switching period.

(b) High load pressure.

Figure 4.11 - The numerical model predicting soft switch piston behavior for the high switching period and load pressure cases. The switched volume pressure is not accurately predicted due to slow unlocked piston movement.
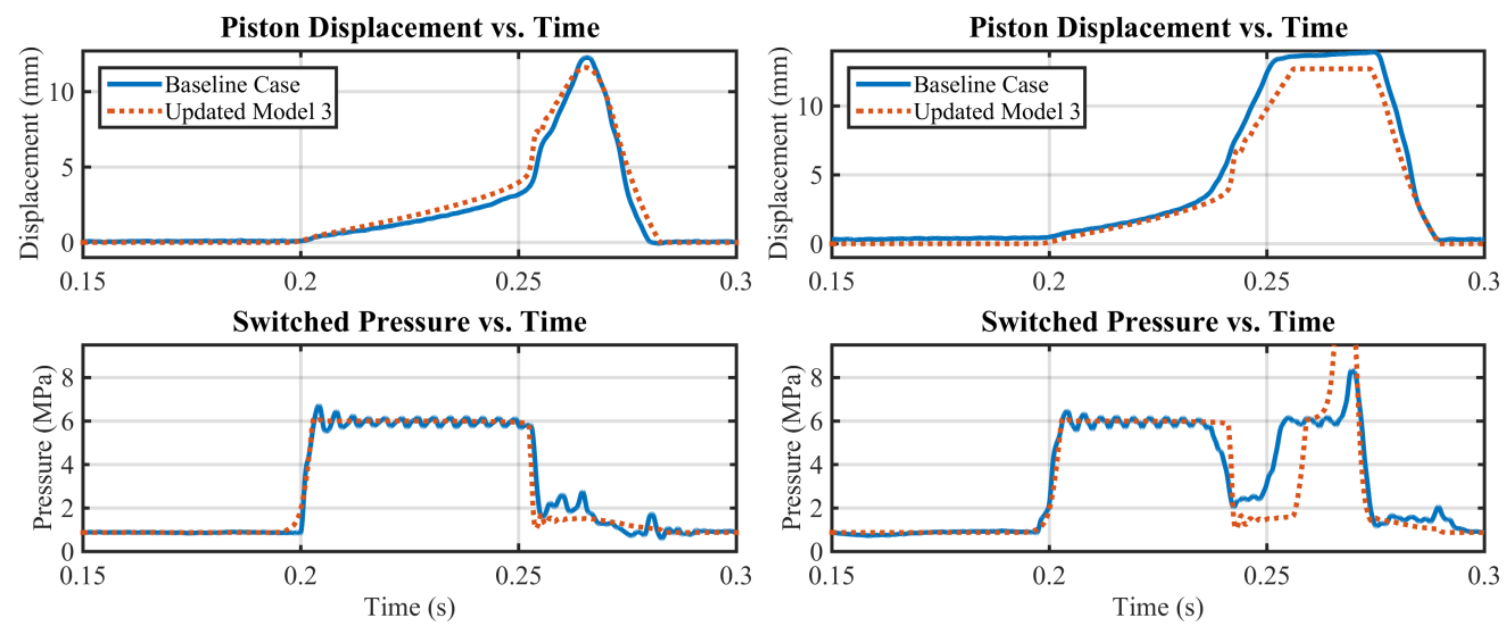

(a) Low soft switch spring preload.

(b) Low soft switch unlocking distance.

Figure 4.12 - The numerical model predicting soft switch piston behavior for the low soft switch spring preload and unlocking distance cases. Like the other cases, the switched volume pressure is not accurately predicted due to slow unlocked piston movement. 


\subsection{System Efficiency}

The purpose of the soft switch is to increase the efficiency of a PWM circuit by removing throttling losses during the control valve transitions. The prototype was tested at the parameters given in Table 4.2, to determine that it operated properly and to find how changes in the system parameters affected the efficiency of the circuit. The efficiency of the prototype at test values was evaluated using Eq. 4.1, with the results shown in Table 4.5. Recall, the control case is the PWM circuit with the soft switch displacement locked, to simulate a lack of soft switch. The baseline soft switch case ended up being less efficient than the control case, indicating that the presence of the soft switch not only did not do anything to increase the system efficiency, but actually decreased it. As the tests shown in Table 4.5 occurred at times where the soft switch was on the edge (or slightly past) failure, it was assumed that the efficiency of each of those cases would be less than that of the baseline case, which is clearly not the case for almost half of the tests. As would be expected, for the cases where the soft switch failed by unlocking early, thereby reaching the piston displacement limit, the system efficiencies were lower than the baseline case. Considering that the control case was more efficient than the baseline case, it also makes sense that the cases where the soft switch failed to unlock were more efficient than the baseline case.

Table 4.5 - The efficiency of the experiment and numerical model at the various test parameters.

\begin{tabular}{llccccccc}
\hline \hline & & \multicolumn{7}{c}{ Parameter Efficiency (\%) } \\
\cline { 3 - 8 } Experiment & Baseline & Control & Period & $\mathbf{P}_{\text {load }}$ & $\mathbf{Q}_{\text {pump }}$ & Unlock & Preload \\
& High & 60.9 & 62.1 & 55.4 & 56.5 & 56.3 & 62.9 & 62.4 \\
& Low & & & 61.3 & 61.5 & 55.1 & 54.0 & 60.5 \\
\hline \multirow{2}{*}{ Num. Model } & High & \multirow{2}{*}{70.5} & \multirow{2}{*}{62.5} & 68.0 & 69.1 & 43.1 & 66.9 & 68.4 \\
& Low & & & 68.9 & 67.7 & 65.0 & 50.5 & 69.8 \\
\hline \hline
\end{tabular}

When looking at the previous soft switch optimization and analysis that was completed by Van de Ven [32], these efficiency results are to be expected. The purpose of this work is to demonstrate the successful operation of the soft switch lock release 
mechanism, therefore the circuit used was one in which the function of the locking soft switch could be isolated from other circuit changes. Van de Ven shows that for the circuit setup used here to test the lock-release concept, the system efficiency is similar to that of a check valve control case, where a check valve is used in place of the load control valve port. A more efficient soft switching approach uses both a passive and a locking soft switch, which is shown to have a potential energy savings of $66 \%$ over a relief valve circuit.

Though the model does a reasonable job predicting the behavior of the soft switch for the baseline and control cases, it appears to be inaccurate in the prediction of system efficiency for those two cases. For the control case, the model predicts $62.5 \%$ efficiency, while for the baseline case an efficiency of $70.5 \%$ is predicted. The control case efficiency is over predicted by $0.4 \%$, which is very close to the expected value, but with the prototype showing that the baseline case is less efficient than the control case, it was unexpected to see the predicted efficiency of the baseline case being $9.6 \%$ higher than testing showed.

Some of the discrepancies in circuit efficiencies between the experimental system and the numerical model may be attributed to the system measurement instruments. The load and switched volume pressures used in the efficiency calculations were determined by transducers that were calibrated by comparison with a calibrated dial gauge with low resolution. The load flow rate was found using a gear meter, which by design have poor detection of small flow pulses, due to gear inertia. Also, the load flow rate used in efficiency calculations is assumed to be simply the flow to load through the control valve, which excludes the effects of flow to and from the back of the soft switch. Since backflow through the check valves behind the soft switch is not well understood, it is difficult to account for these effects on soft switch efficiency calculations.

To provide some context for the above efficiency comparisons, the efficiency of the prototype PWM circuit with soft switch is compared to a simple throttling control circuit as well as a PWM circuit without soft switch. The theoretical throttling valve control strategy efficiency is linear from $0 \%$ efficient at $0 \%$ displacement, to $100 \%$ efficient at 100\% displacement. Neglecting pump inefficiencies, the PWM circuit 
efficiencies are found by the numerical model. For both PWM circuits, the baseline parameter values are used, but to determine the behavior of the PWM circuit without the soft switch, the piston was set to remain at 0 displacement. Also the duty ratio of the control valve signal was adjusted so that, even with control valve delays incorporated, the time that the valve was open to load could be accurately represented. An overlay of the efficiency plots can be seen in Fig. 4.13.

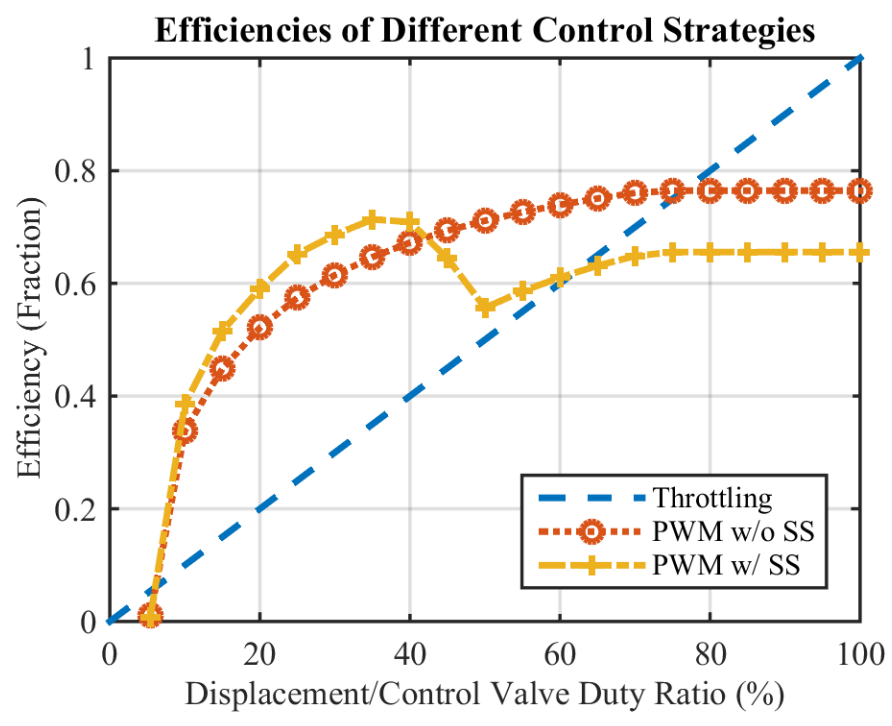

Figure 4.13 - The efficiencies of throttling control, PWM without soft switch, and PWM with soft switch.

As shown in Table 4.4, the delay between the time that the control signal is sent and the control valve starts to transition from tank to load and vice versa is different by approximately 37 milliseconds. The time required for the control valve to complete the transition is also different by approximately 10 milliseconds, depending on the direction of spool travel. These delays and timing differences combine to form the control valve duty ratio, as separate from the command duty ratio.

Notice that as a result of the control valve delays, the PWM circuit efficiencies do not continue to a smooth intersection at zero percent efficiency and zero percent control valve duty ratio. For low displacements, below about 6\%, and high displacements, above about $76 \%$, throttling valve control is theoretically more efficient than both of these 
PWM circuits. The PWM circuit incorporating the soft switch is more efficient than the circuit without soft switch, for control valve duty cycles less than about $42 \%$, at which point the efficiency decreases rapidly before starting to increase again. This decrease in efficiency in the control valve duty range is attributable to the early unlock of the soft switch, which then fails to absorb flow from the switched volume at a time corresponding to the control valve transition to tank. The open soft switch then acts to increase the switched volume and effective compressibility during the switched volume pressure spike.

\subsection{Simulation and Experiment Results Conclusion}

In this chapter, plots showing the soft switch piston displacement and switched volume pressure were presented for an array of parameter adjustments. Comparisons were made between the behavior of the numerical model and prototype soft switches. After updating the model to accurately represent all of the circuit components, it was discovered that major discrepancies still existed between the model and prototype, especially concerning control valve timing and soft switch piston vibration. After adjusting the model in those areas as well as making minor dimension adjustments based on system component measurements, the model was able to predict soft switch piston displacement for the baseline case and cases where the soft switch unlocked early.

The efficiencies of the prototype tests were presented, and it was shown that the efficiency of the soft switch baseline case was lower than the control case. While this was not unexpected, it was also found that the experimentally measured efficiencies were not well predicted by the model. Possible explanations for this, based on the experimental measurement approach, were provided. It was further shown that for control valve duty ratios less than $40 \%$, the PWM circuit used to demonstrate the soft switch mechanism is more efficient than a similar PWM circuit without a soft switch. 


\section{Chapter 5:}

\section{Conclusion}

\subsection{Review}

This thesis describes the theory, design, and experimental validation of a hydraulic soft switch lock release mechanism for use in digital hydraulic systems. Digital hydraulics allow smaller and cheaper fixed displacement pumps to be used in circuits that require variable displacement control. Soft switches in these circuits can minimize control valve transitional throttling losses, the main source of energy loss in PWM circuits, by absorbing flow that would normally be throttled across the transitioning control valve. A lock release mechanism for a soft switch was proposed by Van de Ven that would eliminate the need for an external unlock control [32].

A numerical model was constructed to allow for verification and optimization of the soft switch lock release mechanism. The pressures in front and behind the soft switch are modeled using an effective bulk modulus that accounts for the dependence of bulk modulus on pressure. The port areas for the 3-way control valve and check valves are calculated using lumped port area and discharge coefficients, which allows for the accurate use of the orifice equation to determine system flow rates as a function of the calculated system pressures. Leakage past the piston is assumed to be laminar Couette 
flow, and the soft switch piston and check valve disc positions are determined through integration of force balances.

This model led to the design of a prototype soft switch and circuit with incorporated lock release mechanism. Piston and sleeve material compatibilities were ensured, and the port sizes and other dimensions were designed to allow a larger than necessary soft switch piston displacement. Check valves with appropriate nominal flow rates and cracking pressures were chosen to ensure that pressure signals would be received, and cavitation would not occur. The soft switch circuit was also designed to address air bleeding and ensure accurate system flow and pressure measurements.

Experiments with the soft switch prototype confirmed that under the correct system parameters, the soft switch will unlock and absorb the switched volume pressure spike. Comparisons between the experiments and the numerical model necessitated adjustments to the model based on the actual system components used, especially 3-way control valve timing, and piston and sleeve wear. After these changes were made, the model was able to accurately predict baseline soft switch piston displacement as well as displacement for cases where the soft switch unlocked prematurely. However, the model regularly over predicted soft switch circuit efficiency, probably due to lack of resolution in the measurement approach.

\subsection{Conclusions}

The soft switch unlock concept described by Van de Ven does indeed absorb flow during control valve transitions, minimizing the pressure spike in the circuit switched volume [32]. The range of successful operation in the experimental system is rather small though (Table 4.2), due to excessive leakage past the piston. For changes in load pressure and control valve switching period, the switched volume pressure spikes are much larger than the steady switched volume pressure, indicating that the circuit is especially sensitive to changes in these parameters. As the piston displaces significantly during control valve transitions due to leakage, even cases where the piston does not unlock, the 
switched volume pressure spike is reduced. The reduction of the pressure spike did not increase the system efficiency, as the control case (where the soft switch piston is locked to prevent soft switch function) was experimentally determined to be more efficient than the baseline soft switch case, and most of the other test cases. This phenomenon is addressed in Fig. 4.13, where it is shown that for the dimensions of the experimental model, the soft switch circuit will only be more efficient than a PWM circuit for control valve duty ratios less than about $40 \%$. This decrease in efficiency is due to the early unlock of the soft switch at higher duty ratios, which leads to an increase in switched volume and effective compressibility, and a failure to absorb the switched volume pressure spike.

In tuning the numerical model it was found that two of the most important parameters to understand and control are the gap between the piston and sleeve and the control valve delays and transition profiles. The gap between the piston and sleeve was designed to be between $2.54 \mu \mathrm{m}$ and $17.8 \mu \mathrm{m}$. The initial gap after manufacture was measured at $12.7 \mu \mathrm{m}$, but quickly grew to $31 \mu \mathrm{m}$ due to wear during operation. This large gap allowed the piston to continue to displace to the unlock point when it should have remained fairly stationary in the locked position. The control valve delays, especially the difference in magnitude from one transition delay to the other, were unexpected, leading to more work determining the control valve behavior.

The results of the numerical model in comparison to the experimental model indicate that the model can be used effectively as a future soft switch design tool, especially in the determination of soft switch unlocking behavior. The model over predicts system efficiency under most loading conditions, such as the baseline case, where the numerical model predicted an efficiency almost $10 \%$ higher than calculated from the physical system. The discrepancy is most likely due to the measurement devices failing to capture the dynamic behavior of the experiments, as well as the model not capturing the dynamic flow forces in the 3-way valve and the check valves. Investigating these concerns will do a lot to allow for better accuracy in predictions of circuit efficiency. 


\subsection{Future Work}

In future experimental validation of the unlocking soft switch concept, more care needs to be given to maintaining the gap between the piston and sleeve. Adding multiple lands and grooves around the piston would allow for better centering through pressure equalization. Having a hardened precision ground piston surface would decrease the wear to the piston. Manufacturing the sleeve out of a harder material than bronze would also help reduce wear, though care has to be taken to use compatible materials that will minimize galling of the surfaces.

The distance between the 3-way solenoid control valve and the magnetoresistive position sensor was thought to be large enough that error would not be introduced to the position sensing approach. This oversight increased the complexity and error of the position sensing approach, drastically increasing the preparation and testing time for the various soft switch tests. Using a different approach to determine piston position, such as a laser interferometer, could simplify the approach and would increase the range of design materials, as ferrous materials would no longer need to be avoided out of fear of introducing measurement error.

As mentioned previously, the position of the control valve spool is a source of uncertainty in the model. This uncertainty affects not only soft switch piston displacement, but the efficiency calculations, as errors in piston displacement lead to inaccurate pressure predictions. In future soft switch experimentation, the uncertainty about control valve spool position can be minimized by gathering data about the current flowing through the control valve solenoid, and utilizing the solenoid valve displacement prediction scheme outlined by Yudell [40].

The successful operation range of the soft switch was shown to be fairly small when maintaining flow duty ratio (Table 4.2), but in order for the soft switch to be successfully implemented it needs to be able to successfully operate over a larger range of pressures and duty ratios. This can be achieved by increasing or decreasing the soft switch spring preload and/or spring rate, or changing the piston displacement required to unlock the soft switch. 
To bring the model into better agreement with the experiments, a representation of flow forces through the check valves and 3-way control valve should be included, as well as a better model of control valve spool position. With these updates to the model in place, and the validation of the lock release mechanism, further system optimization can occur. The possibility of extending the soft switch approach to other circuit architectures, including motor and transformer circuits can also be investigated, increasing the efficiencies of a wide range of circuits.

Though the efficiencies calculated for the soft switch prototype do not appear to be much better than a throttling control strategy, it must be remembered that the design objective of the prototype was to validate the soft switch lock release functionality and that it was not optimized for system efficiency. Further, a more efficient use of soft switches in hydraulic PWM circuits involves both a passive and a locking soft switch. Now that the concept has been validated, and the foundation for a design model has been constructed, optimization can occur with a greater level of confidence, with that design moving toward the $66 \%$ energy savings over a relief valve control circuit. 


\section{Bibliography}

[1] Esposito, A., 2009, Fluid Power with Applications, Pearson Education, Upper Saddle River, NJ, Chaps. 2, 5.

[2] Li, P. Y., Li, C. Y., and Chase, T. R., 2005, "Software Enabled Variable Displacement Pumps," ASME International Mechanical Engineering Congress and Exposition, Orlando, FL, November 5-11, ASME Paper No. IMECE2005-81376, pp. 63-72.

[3] Xu, B., Ding, R., Zhang, J., and Su, Q., 2014, "Modeling and Dynamic Characteristics Analysis on a Three-stage Fast-response and Large-flow Directional Valve," Energ. Convers. Manage., 79, pp. 187-199.

[4] Playter, R., Buehler, M., and Raibert, M., 2006, "BigDog," Proceedings of SPIE 6230, Unmanned Systems Technology VIII, 623020.

[5] Love, L. J., 2012, "Estimating the Impact (Energy, Emissions and Economics) of the US Fluid Power Industry," Oak Ridge National Laboratory, December 1.

[6] Zimmerman, J., Pelosi, M., Williamson, C., and Ivantysynova, M., 2007, "Energy Consumption of an LS Excavator Hydraulic System," ASME International Mechanical Engineering Congress and Exposition, Seattle, WA, November 11-15, ASME Paper No. IMECE2007-42267, pp. 117-126.

[7] Williamson, C., Zimmerman, J., and Ivantysynova, M., 2008, "Efficiency Study of an Excavator Hydraulic System Based on Displacement-Controlled Actuators," Bath/ASME Symposium on Fluid Power and Motion Control, Bath, UK, September 10-12, pp. 291-307.

[8] Zimmerman, J., Busquets, E., and Ivantysynova, M., 2011, “40\% Fuel Savings by Displacement Control Leads to Lower Working Temperatures - A Simulation Study and Measurements," Proceedings of the $52^{\text {nd }}$ National Conference on Fluid Power, Las Vegas, NV, March 23-25, NCFP I11-27.2. 
[9] Mohan, N., Undeland, T. M., and Robbins, W. P., 2003, Power Electronics: Converters, Applications, and Design, John Wiley \& Sons, Hoboken, NJ, pp. 161178, Chap. 7.

[10] Rannow, M. B., Tu, H. C., Li, P. Y., and Chase, T. R., 2006, "Software Enabled Variable Displacement Pumps: Experimental Studies," ASME International Mechanical Engineering Congress and Exposition, Chicago, IL, November 5-10, ASME Paper No. IMECE2006-14973, pp. 67-76.

[11] Batdorff, M. A., and Lumkes, J. H., 2006, "Virtually Variable Displacement Hydraulic Pump Including Compressability and Switching Losses," ASME International Mechanical Engineering Congress and Exposition, Chicago, IL, November 5-10, ASME Paper No. IMECE2006-14838, pp. 57-66.

[12] Lumkes, J. H., Batdorff, M. A., and Mahrenholz, J. R., 2009, "Model Development and Experimental Analysis of a Virtually Variable Displacement Pump System," Int. J. Fluid Power, 10(3), pp. 17-27.

[13] Wang F., Gu L., and Chen Y., 2011, "A Continuously Variable Hydraulic Pressure Converter Based on High-Speed On-Off Valves," Mechatronics, 21(8), pp. 12981308.

[14] Edghill, C. M., 1977, "Hydrostatic and Hydrodynamic Transmissions," Engineering Design Guides (Design Council, British Standards Institute and the Council of Engineering Institutions), Oxford University Press, Oxford, UK.

[15] Tomlinson, S. P., and Burrows, C. R., 1992, "Achieving a Variable Flow Supply by Controlled Unloading of a Fixed-Displacement Pump," ASME J. Dyn. Sys., Meas., Control, 114(1), pp. 166-171.

[16] Cao, J., Linyi, G., Wang, F., and Qiu, M., 2005, "Switchmode Hydraulic Power Supply Theory," ASME International Mechanical Engineering Congress and Exposition, Orlando, FL, November 5-11, ASME Paper No. IMECE2005-79019, pp. 85-91.

[17] Beachley, N. H., and Fronczak, F. J., 1987, “A High Efficiency Multi-circuit Sequential Apportioning Hydraulic System," National Conference on Fluid Power, Chicago, Il, March 2-5, pp. 141-152.

[18] Barth, E.J., Zhang, J., and Goldfarb, M., 2003, "Control Design for Relative Stability in a PWM-Controlled Pneumatic System,” ASME J. Dyn. Sys., Meas., Control, 125(3), pp. 504-508.

[19] Flor, M., Scheller, S., and Heidenfelder, R., 2012, "Digital Hydraulics at Bosch Rexroth - A Trend Evolves to Real Applications," The Fifth Workshop on Digital Fluid Power, Tampere, Finland, October 24-25.

[20] Mehling, H., and Weiler, D., 2012, Digital Hydraulics Valve Stage, US patent application US20120286180 A1, November 15. 
[21] De Negri, V. J., Wang, P., Plummer, A., and Johnston, D. N., 2014, "Behavioural Prediction of Hydraulic Step-Up Switching Converters,” Int. J. Fluid Power, 15(1), pp. 1-9.

[22] Guglielmino, E., Semini, C., Kogler, H., Scheidl, R. and Caldwell, D., 2010, "Power Hydraulics - Switched Mode Control of Hydraulic Actuation," IEEE/RSJ International Conference on Intelligent Robots and Systems, Taipei, TW, October 18-22, pp. 3031-3036.

[23] Kudzma, S., Johnston, N., Plummer, A., Sell, N., Hillis, A., and Pan, M., 2012, “A High Flow Fast Switching Valve for Digital Hydraulic Systems," The Fifth Workshop on Digital Fluid Power, Tampere, Finland, October 24-25.

[24] Katz, A. A., and Van de Ven, J. D., "Design of a High-Speed On-Off Valve," ASME International Mechanical Engineering Congress and Exposition, Lake Buena Vista, FL, November 13-19, ASME Paper No. IMECE2009-11189, pp. 237-246.

[25] Katz, A., 2008, "Design of a High Speed Hydraulic On/Off Valve," M.S. Thesis, Worcester Polytechnic Institute, Worcester, MA, May 28.

[26] Van de Ven, J. D., and Katz, A., 2011, "Phase-Shift High-Speed Valve for SwitchMode Control,” ASME J. Dyn. Sys., Meas., Control, 133(1), p. 011003.

[27] Wu, J. J., and Van de Ven, J. D., 2010, "Development of a High-Speed On-Off Valve for Switch-Mode Control of Hydraulic Circuits with Four-Quadrant Control," ASME International Mechanical Engineering Congress and Exposition, Vancouver, British Columbia, Canada, November 12-18, ASME Paper No. IMECE2010-37380, pp. 281-288.

[28] Tu, H. C., Rannow, M. B., Van de Ven, J. D., Wang, M., Li, P. Y., and Chase, T. R., 2007, "High Speed Rotary Pulse Width Modulated On/Off Valve," ASME International Mechanical Engineering Congress and Exposition, Seattle, WA, November 11-15, ASME Paper No. IMECE2007-42559, pp. 89-102.

[29] Tu, H. C., Rannow, M. B., Wang, M., Li, P. Y., Chase, T. R., and Van de Ven, J. D., 2012, "Design, Modeling, and Validation of a High-Speed Rotary Pulse-WidthModulation On/Off Hydraulic Valve," ASME J. Dyn. Sys., Meas., Control, 134(6), p. 061002.

[30] Rannow, M. B., and Li, P. Y., 2012, "Soft Switching Approach to Reducing Transition Losses in an On/Off Hydraulic Valve," ASME J. Dyn. Sys., Meas., Control, 134(6), p. 064501.

[31] Vorperian, V., 1988, "Quasi-Square Wave Converters: Topologies and Analysis," IEEE Transactions on Power Electronics, 3(2), pp. 183-191.

[32] Van de Ven, J., 2014, "Soft Switch Lock-Release Mechanism for a Switch-Mode Hydraulic Pump Circuit,” ASME J. Dyn. Sys., Meas., Control, 136(3), p. 031003. 
[33] Triana, D., 2014, "Hydraulic Locking Soft Switch Mechanism for Switch-Mode Hydraulics: Design and Experimental Validation," University of Minnesota Department of Mechanical Engineering, Minneapolis, MN, May 6.

[34] Van de Ven, J., 2012, "On Fluid Compressibility in Switch-Mode Hydraulic Circuits - Part I: Modeling and Analysis,” ASME J. Dyn. Sys., Meas., Control, 135(2), p. 021013.

[35] Gholizadeh, H., Burton, R., and Schoenau, G. J., 2012, "Fluid Bulk Modulus: Comparison of Low Pressure Models," Int. J. Fluid Power, 13(1), pp. 7-16.

[36] Cho, B.-H., Lee, H.-W., and Oh, J.-S., 2002, "Estimation Technique of Air Content in Automatic Transmission Fluid by Measuring Effective Bulk Modulus," KSAE Int. J. Autom. Technol., 3(2), pp. 57-61.

[37] Çengel, Y. A., and Cimbala, J. M., 2010, Fluid Mechanics: Fundamentals and Applications, McGraw-Hill, New York, NY, Chaps. 5, 10.

[38] Chapra, S. C., and Canale, R. P., 2006, Numerical Methods for Engineers, McGraw-Hill, New York, NY, pp. 16-17, Chap. 1.

[39] International Organization for Standardization, 1992, Industrial Liquid Lubricants ISO Viscosity Classification, ISO 3448:1992.

[40] Yudell, A. C., and Van de Ven, J. D., "Predicting Solenoid Valve Spool Displacement Through Current Analysis," Int. J. Fluid Power, under review.

[41] Van de Ven, J., 2012, "On Fluid Compressibility in Switch-Mode Hydraulic Circuits - Part II: Experimental Results,” ASME J. Dyn. Sys., Meas., Control, 135(2), p. 021014. 
Appendices 


\section{Appendix A:}

\section{Detail Design of Soft Switch}

This appendix contains the detail design for each of the four components of the soft switch that needed to be manufactured for soft switch testing. 


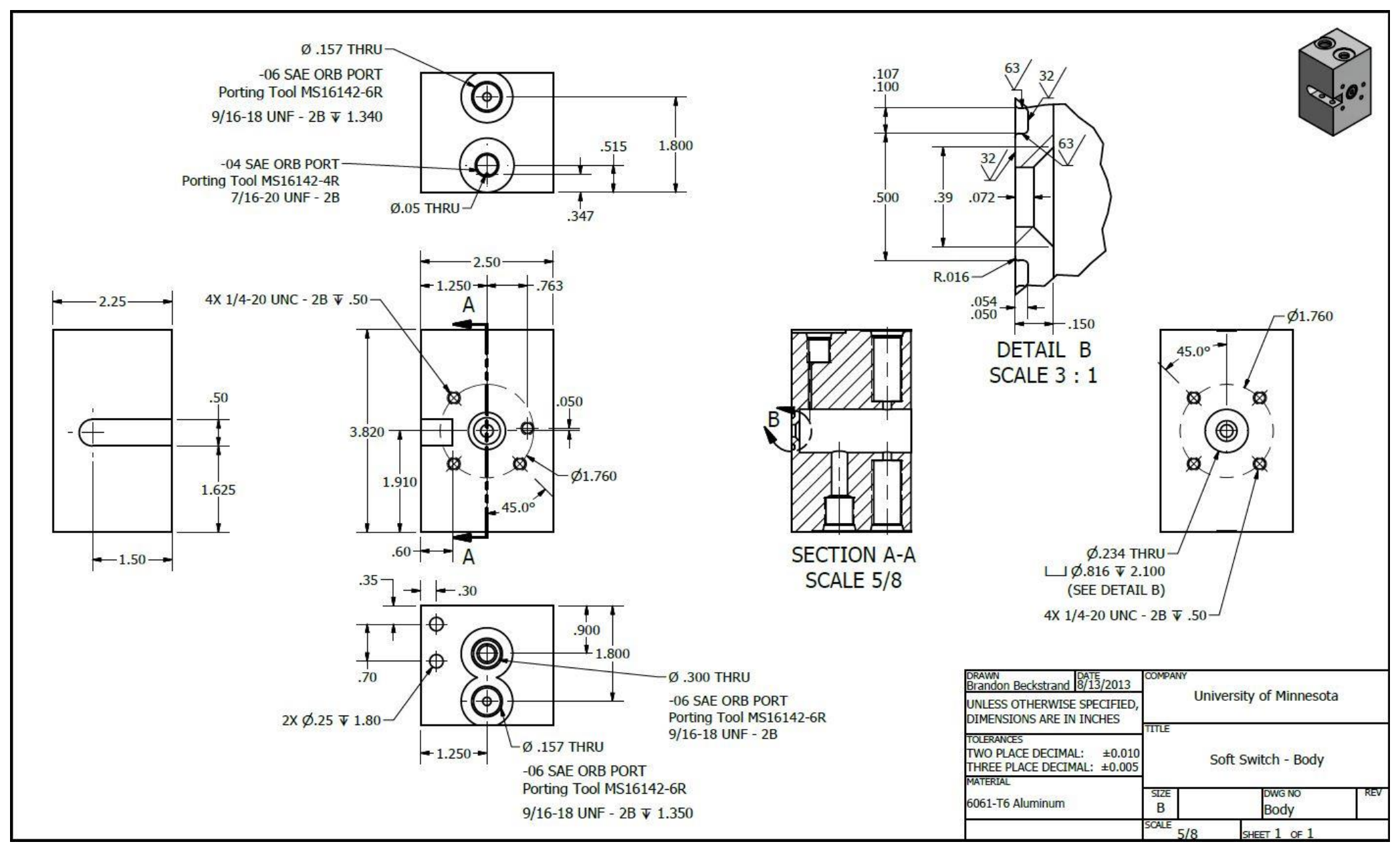

Figure A.1 - Detail design for the soft switch manifold. 


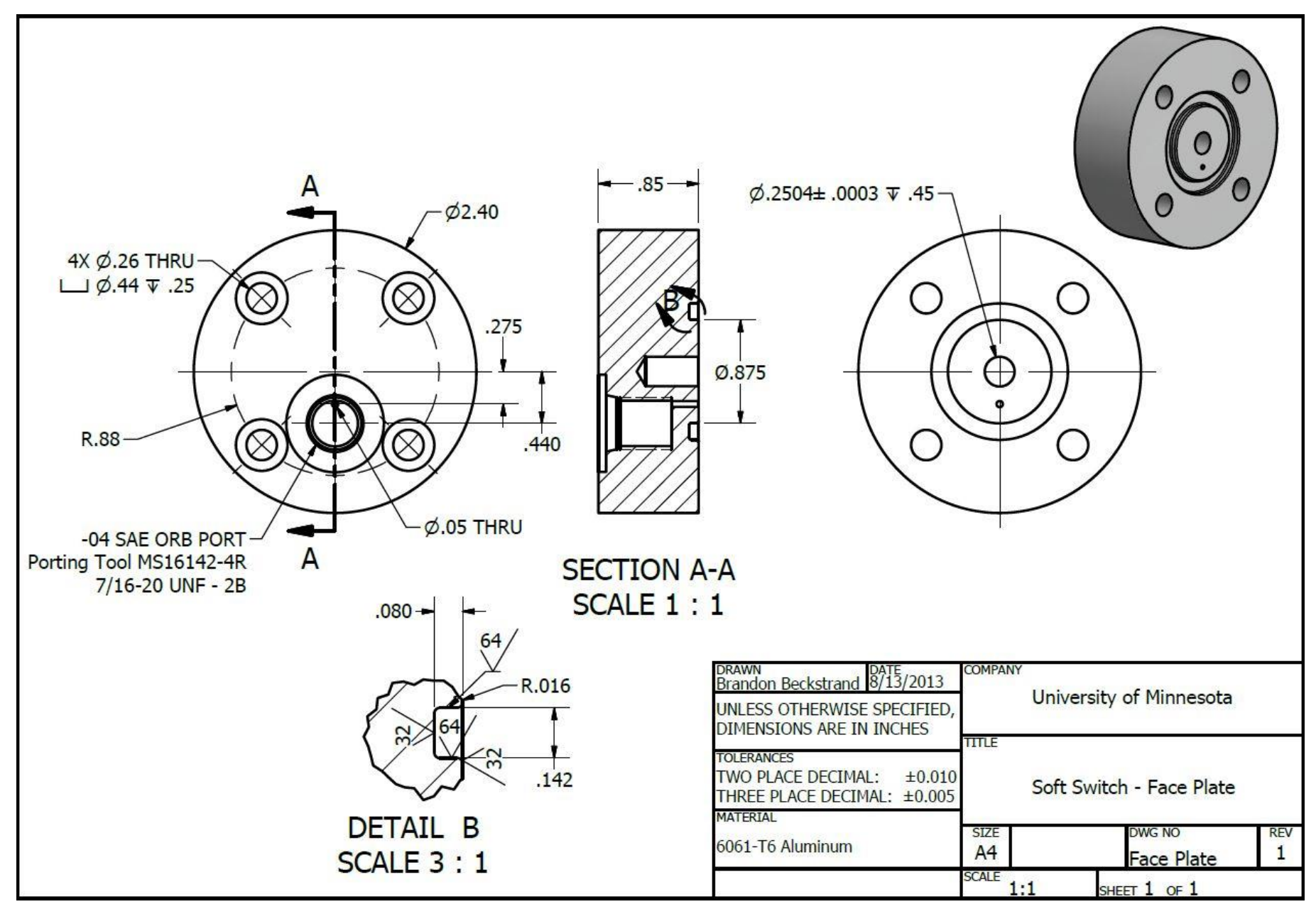

Figure A.2 - Detail design for the soft switch face plate. 


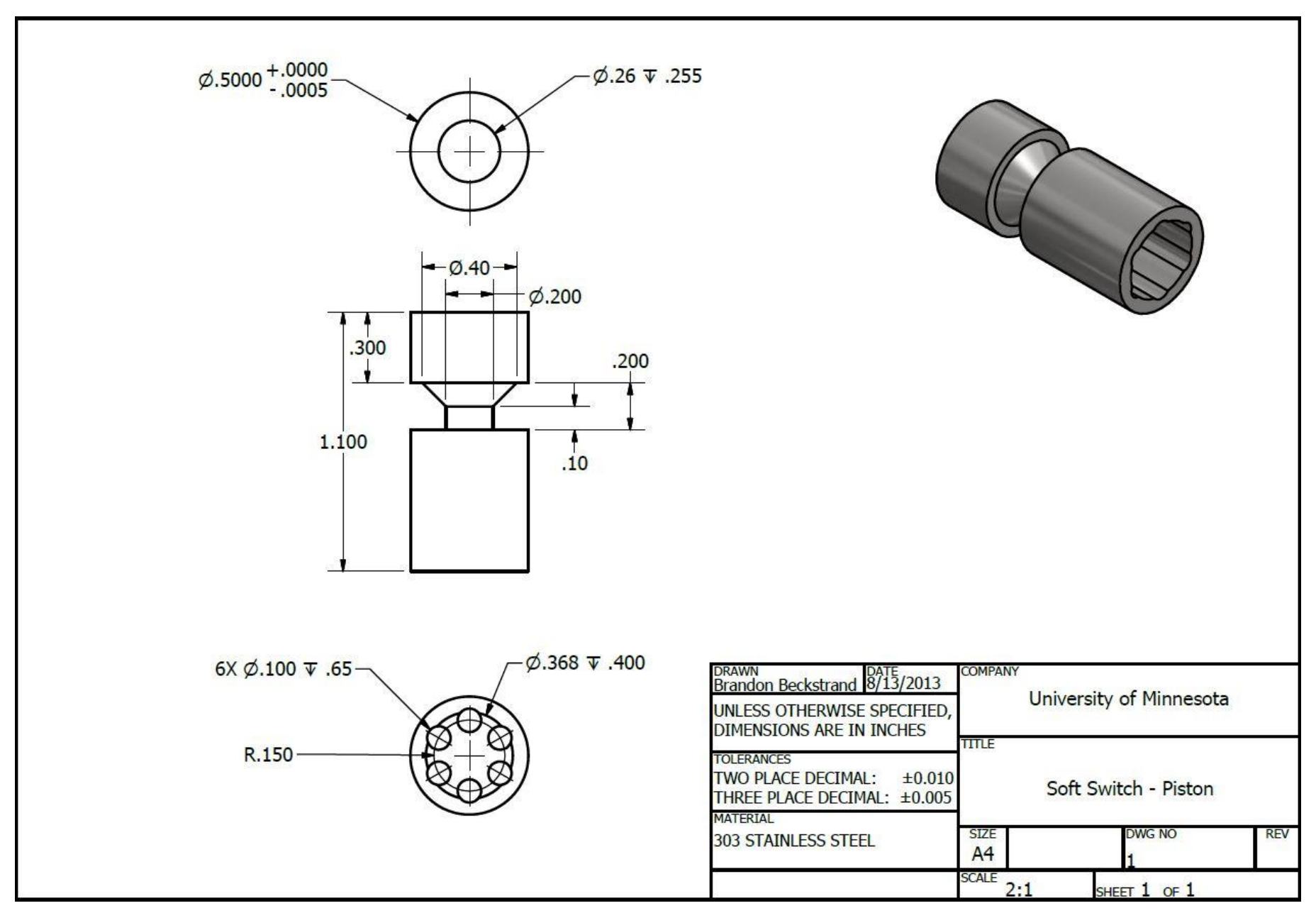

Figure A.3 - Detail design for soft switch piston. 


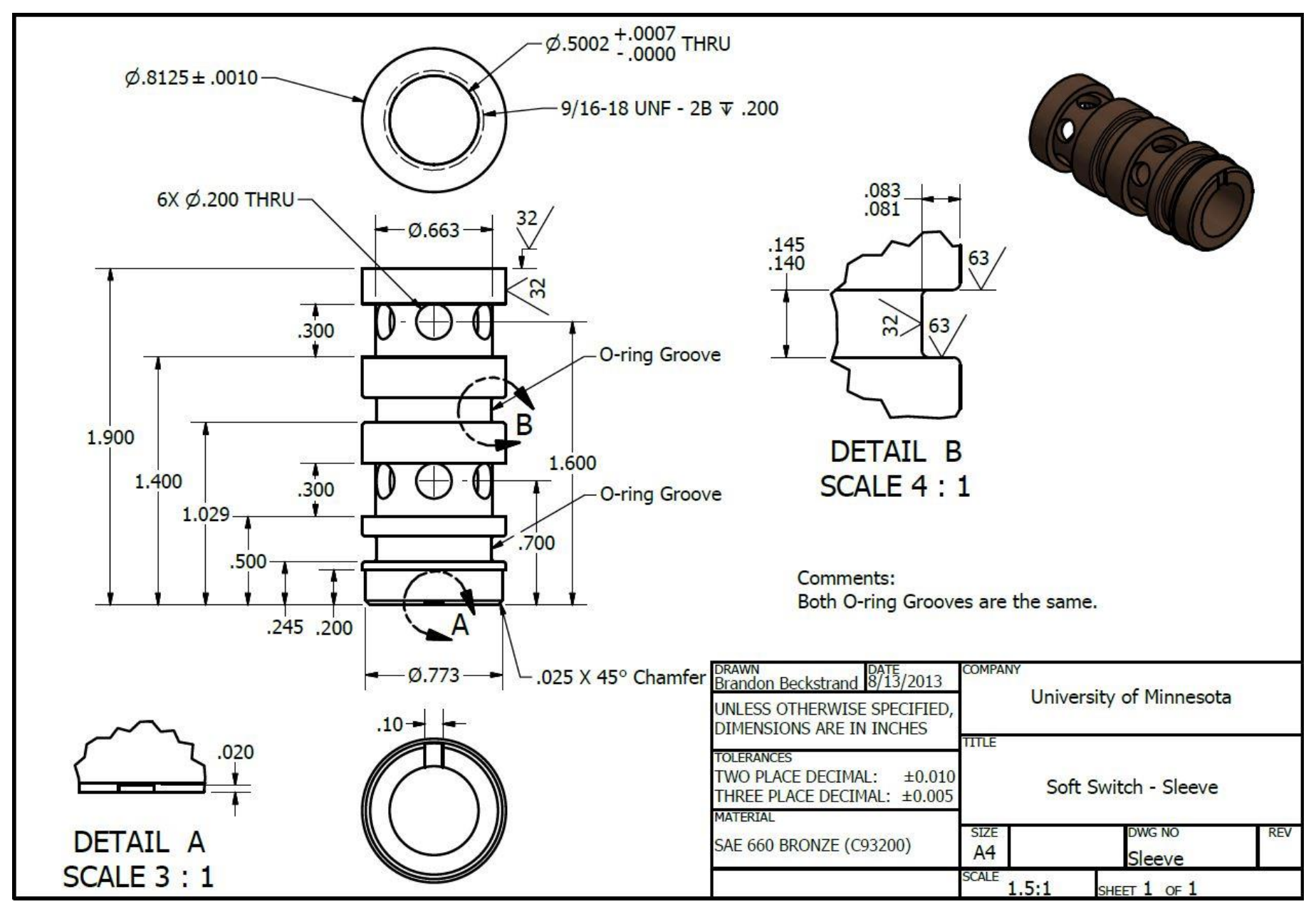

Figure A.4 - Detail design for soft switch sleeve. 


\section{Appendix B:}

\section{Matlab Files}




\section{B.1 Position Sensor Calibration}

The position sensor calibration was done in four parts. The first part (DAQ - Position Sensor Offset) helped determine if the position sensor was placed at an appropriate location along the axis of the soft switch, so that clear unique data could be received for all piston displacements. It also found the maximum and minimum sensor values so that a proper offset could be determined.

$\% \%$ DAQ - Position Sensor Offset

$\%$ MEPS Laboratory

$\%$ Brandon Beckstrand

$\% 17$ May 2014

$\% \%$ Program Description

$\%$ This program is used to find the proper offset and max and min values of the sensor output so the two signals can be combined and curve fit.

$\%$ The pump needs to be on and the piston needs to be able to travel to the extremes for this code.

$\% 1$ of 4 Position Sensor Calibration Codes

$\% \%$ Variable Creation

$\%$ Test Parameters

sampleRate $=10000$

totalTime $=20$;

$\mathrm{T}=10$

duty_1 $=0.5$;

duty = 1 - duty_1;

$\%$ Create 3-way Valve Output Vector

out $=5 *$ ones $(($ sampleRate $*$ totalTime $)-1,1)$;

out $($ sampleRate $*$ totalTime, 1$)=0$;

for $\mathrm{i}=1$ :(totalTime/T) -1

for $\mathrm{j}=1$ :sampleRate $* \mathrm{~T}^{*}$ duty_1

out $($ round $($ sampleRate $* \mathrm{~T} * \mathrm{i}+\mathrm{j}), 1)=0$;

end

end

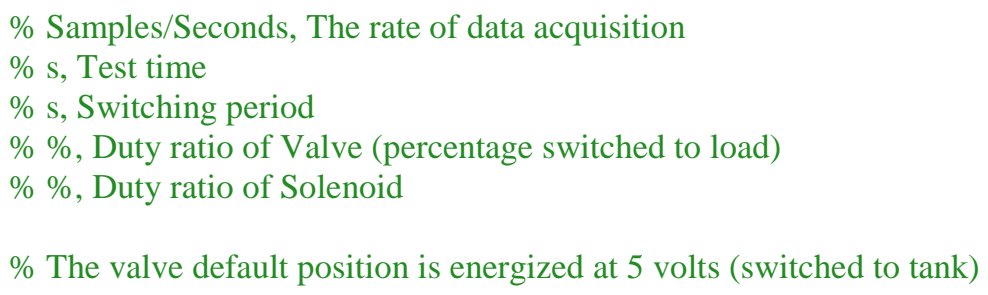

$\%$ The valve default position is energized at 5 volts (switched to tank) 
$\% \%$ Run DAQ

$\%$ Create DAQ Session

device $=$ daq.getDevices;

$\mathrm{s}=$ daq.createSession('ni');

s. Rate = sampleRate;

s.DurationInSeconds = totalTime;

$\%$ Sensor Channels

ch1=s.addAnalogInputChannel('Dev1', 'ai6', 'Voltage');

ch2=s.addAnalogInputChannel('Dev1', 'ai7', 'Voltage');

$\%$ Define Sensor Input Type

s.Channels(1).InputType = 'Differential';

s.Channels(2).InputType $=$ 'Differential';

$\%$ Output Channels (Valve Input)

ch3=s.addAnalogOutputChannel('Dev1', 'ao2', 'Voltage'); \% 3-way Valve Input

s.queueOutputData(out);

$\%$ Start Test and Collect Data

$[$ data, $\mathrm{t}]=\mathrm{s}$.startForeground();

s.wait();

s.release();

delete(s);

$\% \%$ Convert Data and Create Plots

$\%$ Filter data

data(:,1) = filter $500 \_1000 \mathrm{~Hz}($ data $(:, 1))$

data $(:, 2)=$ filter_500_1000Hz(data(:,2));

$\%$ Preallocate Matrices

data_offset $=$ zeros $($ length $($ data $(:, 1)), 2)$;

theta $=$ zeros(length(data(:,1)),1);

$\%$ Determine the Current Max and Min Values of the Sensor Outputs

$\max A=\max (\operatorname{data}(2000:$ length $(\operatorname{data}(:, 1)), 1))$;

$\min A=\min (\operatorname{data}(2000:$ length $(\operatorname{data}(: 1)), 1))$;

$\max B=\max (\operatorname{data}(2000:$ length $($ data $(:, 2)), 2))$

$\min B=\min (\operatorname{data}(2000:$ length $(\operatorname{data}(:, 2)), 2))$;

$\%$ Determine the Max and Min Values of the Sensor Outputs after Offsets are Applied

p_pA $=\max A-\min A$

$\mathrm{p} \_\mathrm{pB}=\max \mathrm{B}-\min \mathrm{B}$

offset $\mathrm{A}=\left(\mathrm{p} \_\mathrm{pA} / 2\right)-\max \mathrm{A}$;
$\%$ Device is a variable that lists N.I. devices that are available

$\%$ Creates a session object using an N.I. product (that was found in the previous step)

$\%$ Number of samples taken every second

$\%$ The duration of the test

$\%$ Position Sensor A

$\%$ Position Sensor B

$\%$ Position Sensor A

$\%$ Position Sensor B

$\%$ Starts operations of session object "s"

$\%$ Blocks matlab until all background objects complete (session object "s")

$\%$ Releases the hardware (not necessary in this use)

$\%$ Deletes the session object hardware 
offsetB $=\left(p \_p B / 2\right)-\operatorname{maxB}$

newmax $\mathrm{A}=\max \mathrm{A}+\operatorname{offset} \mathrm{A}$

newmax $\mathrm{B}=\max \mathrm{B}+$ offsetB;

$\%$ Offset and Scale the data

data_offset(:,1) = data $(:, 1)+$ offsetA;

data_offset(:,2) = data $(:, 2)+$ offsetB;

The second part (DAQ - Position Sensor Calibration Point) gathered position sensor data for known piston displacements for later curve fitting.

$\% \%$ DAQ - Position Sensor Calibration Point

$\%$ MEPS Laboratory

$\%$ Brandon Beckstrand

$\% 17$ May 2014

$\% \%$ Program Description

$\%$ This program is used to find the data points required to do a curve fit of the magnetic field angle vs piston position.

$\%$ The pump needs to be turned off and the 3-way valve need to be left alone (flow going to load).

$\% 2$ of 4 Position Sensor Calibration Codes

$\% \%$ Variable Creation

$\%$ Test Parameters

sampleRate $=10000$

totalTime $=2$

$\mathrm{a}=1$;

$\% \%$ Run DAQ

$\%$ Create DAQ Session

device $=$ daq.getDevices;

$\mathrm{s}=$ daq.createSession('ni')

s.Rate = sampleRate;

s.DurationInSeconds = totalTime;

$\%$ Samples/Seconds, The rate of data acquisition

$\%$ s, Test time

$\%$ N/A, Number of acrylic standoffs +1 (1 to 6$)$

$\%$ Sensor Channels

ch1=s.addAnalogInputChannel('Dev1', 'ai6', 'Voltage'); \% Position Sensor A

ch2=s.addAnalogInputChannel('Dev1', 'ai7', 'Voltage'); \% Position Sensor B

$\%$ Define Sensor Input Type 


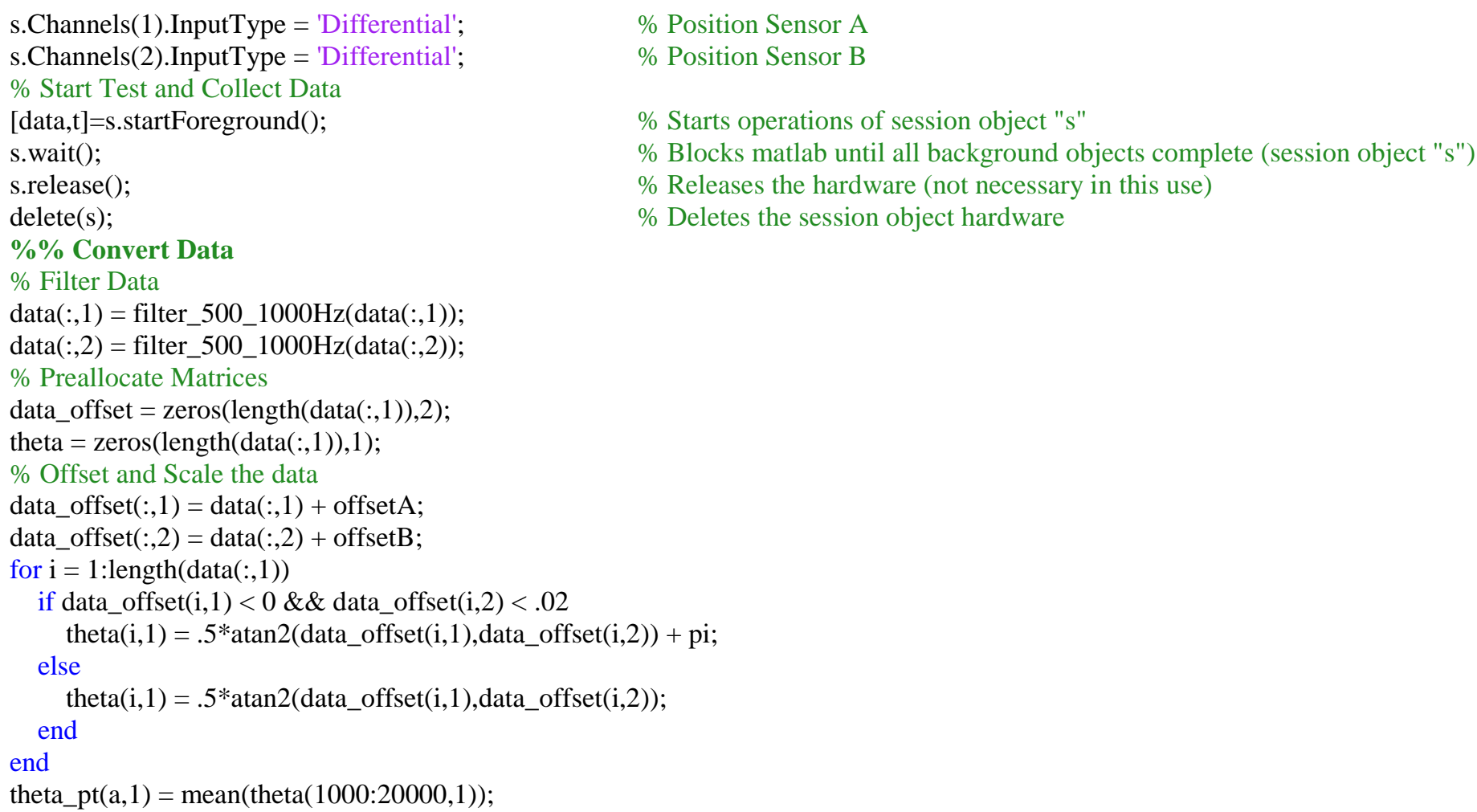

$\%$ Starts operations of session object "s"

$\%$ Blocks matlab until all background objects complete (session object "s")

$\%$ Releases the hardware (not necessary in this use)

$\%$ Deletes the session object hardware

The third part (DAQ - Position Sensor Curve Fit) does a simple curve fit on the position sensor data previously gathered.

$\% \%$ DAQ - Position Sensor Curve Fit

$\%$ MEPS Laboratory

$\%$ Brandon Beckstrand

$\% 17$ May 2014

$\% \%$ Program Description

$\%$ This program takes the data found in "DAQ - Position Sensor Calibration Point" and curve fits it to relate magnetic field angle and piston position.

$\% 3$ of 4 Position Sensor Calibration Codes

$\%$ \% Run Curve Fit Program 
polyX $=\left[\right.$ theta_pt $(1,1)$, theta_pt $(2,1)$, theta_pt $(3,1)$, theta $\_p t(4,1), \ldots$

theta_pt $(5,1)$, theta_pt $(6,1)]$;

polyY $=[0, .114, .228, .342, .456, .57]$

position $(:, 1)=\operatorname{polyfit}(\operatorname{polyX}$, polyY, 3$)$;

The fourth and final part (DAQ - Position Sensor Solenoid Correction Calculation) is used to build a database of solenoid error sensed by the position sensor as a function of period and duty ratio.

$\% \%$ DAQ - Position Sensor Solenoid Correction Calculation

$\%$ MEPS Laboratory

$\%$ Brandon Beckstrand

$\% 17$ May 2014

$\% \%$ Program Description

$\%$ This program is used to create the profile required to remove the effects of the high speed valve solenoid from the position sensor data.

$\%$ The program should be run when the pump is off and the Soft Switch Piston is at zero displacement.

$\% 4$ of 4 Position Sensor Calibration Codes

$\% \%$ Variable Creation

clear all

load('C: IUsers lbecks033\Desktop\Code 1-4\Code 1-3.mat');

\% Test Parameters

sampleRate $=10000$

totalTime $=1$

$\mathrm{T}=0.162$;

duty_l $=0.75$;

duty = 1 - duty_l;

look_duty = num2str(duty_1);

look_T = num2str(T);

$\% \%$ Clear required variables

clear data

clear data_offset

$\% \%$ Run DAQ

$\%$ Create DAQ Session

device $=$ daq.getDevices;

$\mathrm{s}=$ daq.createSession('ni')

$\%$ Device is a variable that lists N.I. devices that are available

s. Rate $=$ sampleRate

$\%$ Samples/Seconds, The rate of data acquisition

$\%$ s, Test time

$\% \mathrm{~s}$, Switching period

$\% \%$, Duty ration of Valve (percentage switched to load)

$\% \%$, Duty ratio of Solenoid

$\%$ Creates a session object using an N.I. product (that was found in the previous step)

$\%$ Number of samples taken every second 
s.DurationInSeconds = totalTime;

$\%$ Sensor Channels

ch1=s.addAnalogInputChannel('Dev1', 'ai3', 'Voltage'); ch2=s.addAnalogInputChannel('Dev1', 'ai6', 'Voltage');

ch3=s.addAnalogInputChannel('Dev1', 'ai7', 'Voltage');

$\%$ Define Sensor Input Type

s.Channels(1).InputType = 'Differential';

s.Channels(2).InputType = 'Differential';

s.Channels(3).InputType $=$ 'Differential';

$\%$ Start Test and Collect Data

[data, $\mathrm{t}]=\mathrm{s}$.startForeground();

s.wait();

s.release();

delete(s);

$\% \%$ Create Correction Curve

for $\mathrm{i}=2$ :length(data(:,1))

if $\operatorname{data}(\mathrm{i}, 1)<2$

if data $(\mathrm{i}-1,1)>2$

trunc $=\mathrm{i}$;

break

end

end

end

for $\mathrm{i}=1: 3$

trunca $(:, 1)=\operatorname{data}($ trunc:end, $\mathrm{i})$;

truncb $(:, 1)=$ zeros $($ trunc $-1,1)$;

dataTrunc $(:, \mathrm{i})=[$ trunca;truncb $]$;

end

$\%$ Filter Data

data_filter(:,1) = dataTrunc $(:, 1)$;

data_filter(:,2) = filter_500_1000Hz(dataTrunc $(:, 2))$;

data_filter(:,3) = filter_500_1000Hz (dataTrunc $(:, 3))$;

$\%$ Preallocate matrix

en_cor_vect $=$ zeros $($ sampleRate $*$ totalTime, 1$)$;

theta $=$ zeros(sampleRate*totalTime, 1 );

$\%$ Offset and Scale the data
$\%$ The duration of the test

$\%$ High Speed Valve Signal

\% Position Sensor A

Position Sensor B

$\%$ High Speed Valve Signal

$\%$ Position Sensor A

$\%$ Position Sensor B

$\%$ Starts operations of session object "s"

\% Blocks matlab until all background objects complete (session object "s")

$\%$ Releases the hardware (not necessary in this use)

$\%$ Deletes the session object hardware 
data_offset $(:, 1)=$ data_filter $(:, 2)+$ offsetA;

data_offset $(:, 2)=$ data_filter(:,3) + offsetB;

$\%$ Combine the Sensor Output Vectors

for $\mathrm{i}=1$ :length $($ data $(:, 2))$

if data_offset $(\mathrm{i}, 1)<0$ \&\& data_offset $(\mathrm{i}, 2)<.02$

theta $(i, 1)=.5 * \operatorname{atan} 2($ data_offset $(i, 1)$,data_offset $(i, 2))+$ pi

else

theta $(i, 1)=.5^{*} \operatorname{atan} 2($ data_offset $(i, 1)$,data_offset $(i, 2))$;

end

end

$\%$ Determine Zero Displacement Sensor Output

$\operatorname{mina}=\min \left(\operatorname{theta}\left(200: 200+\operatorname{round}\left(\mathrm{T}^{*}\right.\right.\right.$ sampleRate $\left.\left.), 1\right)\right)$;

$\operatorname{minb}=\min \left(\operatorname{theta}\left(200+\operatorname{round}\left(\mathrm{T}^{*}\right.\right.\right.$ sampleRate $): 200+\operatorname{round}(\mathrm{T} *$ sampleRate $\left.\left.* 2), 1\right)\right)$;

$\operatorname{minc}=\min \left(\operatorname{theta}\left(200+\operatorname{round}\left(\mathrm{T}^{*}\right.\right.\right.$ sampleRate*2):200+round $\left(\mathrm{T}^{*}\right.$ sampleRate*3),1)$)$;

mean $=($ mina $+\operatorname{minb}+\operatorname{minc}) / 3$;

$\%$ Place Zero Displacement at Zero

en_offset $(:, 1)=\operatorname{theta}(:, 1) ; \%$ - mean;

clear mean;

$\%$ Create Correction Vector for Each Sensor Output

en_cor_vect $1(:, 1)=$ en_offset(round $\left(\mathrm{T}^{*}\right.$ sampleRate $)+1$ :round $\left(2 * \mathrm{~T}^{*}\right.$ sampleRate $\left.)-3,1\right)$;

en_cor_vect $2(:, 1)=\operatorname{repmat}($ en_cor_vect $1(:, 1), \operatorname{round}($ totalTime/T) $-1,1)$;

for $\mathrm{i}=1$ :length(en_cor_vect $2(:, 1))$

en_cor_vect $(i, 1)=$ en_cor_vect $2(i, 1)$;

end

save(['C:IUsers \becks033\Desktop\Code 1-4l','Code 1-4-',look_duty,'-',look_T,'.mat']); 


\section{B.2 Three-way Valve Control and Data Acquisition}

The 3-way valve was controlled separately from the test data acquisition. DAQ - Soft Switch Circuit 3-way Valve Control was used to send an output vector to the valve solenoid.

$\% \%$ DAQ - Soft Switch Circuit 3-way Valve Control

$\%$ MEPS Laboratory

$\%$ Brandon Beckstrand

$\% 17$ May 2014

$\% \%$ Program Description

$\%$ This program is for control of the Soft Switch 3-way Valve.

$\% \%$ Variable Creation

$\%$ Test Parameters

sampleRate $=10000$;

totalTime $=1000$

$\mathrm{T}=0.15$;

duty_1 $=0.52$;

duty = 1 - duty_1;

$\%$ Create 3-way Valve Output Vector

out $=5 *$ ones $(($ sampleRate $*$ totalTime $)-1,1)$;

out $($ sampleRate $*$ totalTime, 1$)=0$;

for $\mathrm{i}=1$ :(totalTime/T) -1

for $\mathrm{j}=1$ :sampleRate*T*duty_1

out $($ round $($ sampleRate $* \mathrm{~T} * \mathrm{i}+\mathrm{j}), 1)=0$; end

end

$\% \%$ Run DAQ

$\%$ Create DAQ Session

device $=$ daq.getDevices;

$\mathrm{s}=$ daq.createSession('ni');

$\%$ Samples/Seconds, The rate of data acquisition

$\%$ s, Test time

$\%$ s, Switching period

$\% \%$, Duty ration of Valve (percentage switched to load)

$\% \%$, Duty ratio of Solenoid

$\%$ The valve default position is energized at 5 volts (switched to tank)

$\%$ Make sure the valve isn't energized after the test
$\%$ Device is a variable that lists N.I. devices that are available

$\%$ Creates a session object using an N.I. product (that was found in the previous step) 
s.Rate = sampleRate;

s.DurationInSeconds = totalTime;

$\%$ Output Channels (Valve Input)

ch1=s.addAnalogOutputChannel('Dev1', 'ao3', 'Voltage'); \% 3-way Valve Input

s.queueOutputData(out);

$\%$ Start Test and Collect Data

$[$ data, $\mathrm{t}]=\mathrm{s}$.startForeground () ;

s.wait();

s.release () ;

delete(s);
$\%$ Number of samples taken every second

$\%$ The duration of the test

The data acquisition was completed by DAQ - Soft Switch Circuit. This code gathered data from three pressure transducers, two flow meters, the position sensor, a thermocouple (placed in the reservoir), and the 3-way valve actuation signal from DAQ - Soft Switch Circuit 3-way Valve Control. With the control valve actuation signal being acquired, the test data could then accurately be aligned with the 3-way valve signal and analyzed.

$\% \%$ DAQ - Soft Switch Circuit

$\%$ MEPS Laboratory

$\%$ Brandon Beckstrand

\% 17 May 2014

$\% \%$ Program Description

$\%$ This program is used for data acquisition for the Soft Switch.

$\%$ Data is gathered from the following:

$\% \quad$ Three pressure transducers

$\% \quad$ High Speed Valve Actuation Signal

$\% \quad$ Two flow meters

$\% \quad$ One position sensor

$\% \quad$ Tank Temperature

$\% \%$ Variable Creation

$\%$ Test Parameters

sampleRate $=10000$;

total Time $=1$;

$\%$ Starts operations of session object "s"

$\%$ Blocks matlab until all background objects complete (session object "s")

$\%$ Releases the hardware (not necessary in this use)

$\%$ Deletes the session object hardware

$\mathrm{T}=0.15$;

$\%$ Samples/Seconds, The rate of data acquisition

$\%$ s, Test time

$\% \mathrm{~s}$, Switching period 
duty_l $1=0.52$

duty = 1 - duty_l;

\% Load the correct Solenoid Disturbance removal data

look_duty = num2str(duty_l);

look_T = num 2 str( $(\mathrm{T})$;

load(['C:IUsers lbecks033〉Desktop\Code 1-4l','Code 1-4-',look_duty,'-',look_T,'.mat']);

$\% \%$ Run DAQ

$\%$ Create DAQ Session

device $=$ daq.getDevices;

$\mathrm{s}=$ daq.createSession('ni');

s. Rate = sampleRate;

s.DurationInSeconds = totalTime;

$\%$ Sensor Channels

ch1=s.addAnalogInputChannel('Dev1', 'ai0', 'Voltage'); \% Switched Volume Pressure

ch2=s.addAnalogInputChannel('Dev1', 'ai1', 'Voltage'); \% Pressure Behind Soft Switch

ch3=s.addAnalogInputChannel('Dev1', 'ai2', 'Voltage'); \% Load Pressure

ch4=s.addAnalogInputChannel('Dev1', 'ai3', 'Voltage'); \% High Speed Valve Signal

ch5=s.addAnalogInputChannel('Dev1', 'ai4', 'Voltage'); \% Pump Line Flow Rate (Coriolis)

ch6=s.addAnalogInputChannel('Dev1', 'ai5', 'Voltage'); \% Load Line Flow Rate (Gear)

ch7=s.addAnalogInputChannel('Dev1', 'ai6', 'Voltage'); \% Position Sensor A

ch8=s.addAnalogInputChannel('Dev1', 'ai7', 'Voltage'); \% Position Sensor B

ch9=s.addAnalogInputChannel('Dev1', 'ai24', 'Voltage'); \% Tank Temperature

$\%$ Define Sensor Input Type

s.Channels(1).InputType = 'Differential';

s.Channels(2).InputType = 'Differential';

s.Channels(3).InputType = 'Differential';

s. Channels(4).InputType $=$ 'Differential';

s.Channels(5).InputType = 'Differential';

s.Channels(6).InputType = 'Differential';

s. Channels(7).InputType $=$ 'Differential';

s.Channels(8).InputType = 'Differential';

s.Channels(9).InputType = 'SingleEnded';

$\%$ Start Test and Collect Data

[data,t]=s.startForeground();

s.wait();

s.release();

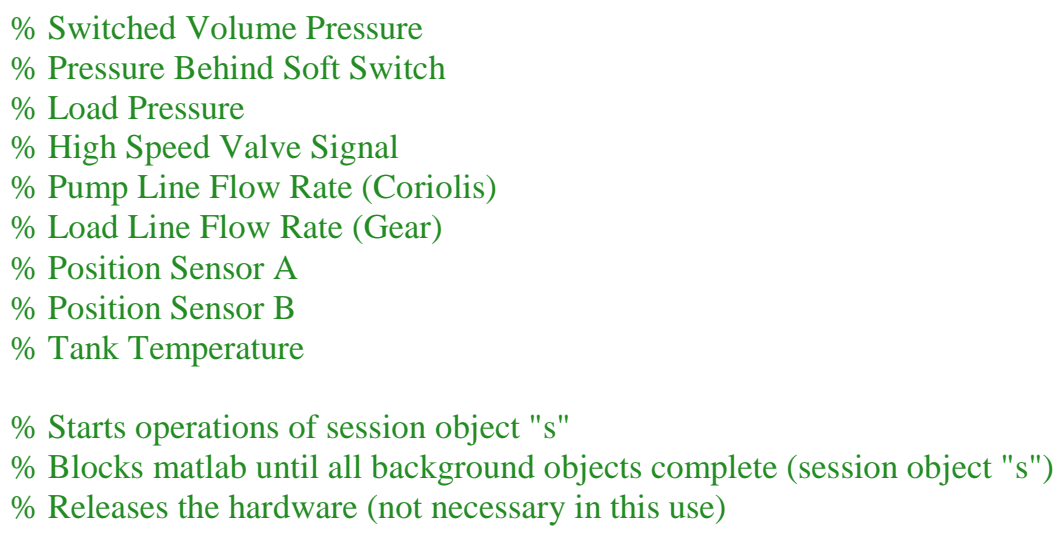

$\%$ Releases the hardware (not necessary in this use) 
